\title{
Data report: paleomagnetism and rock magnetism of sediments from offshore Canterbury Basin, IODP Expedition 317
}

\author{
Jaume Dinarès-Turell ${ }^{2}$ and Kirsty Tinto ${ }^{3,4}$
}

\section{Chapter contents}

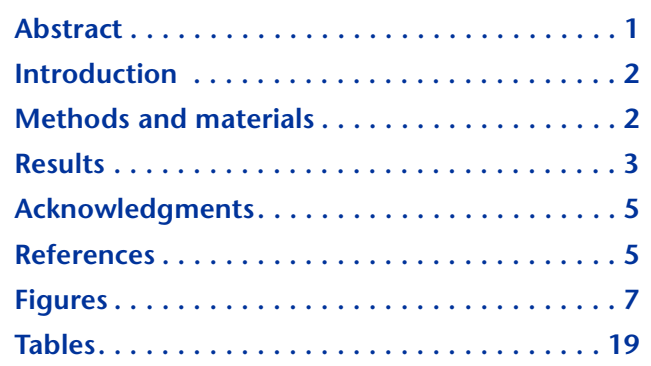

${ }^{1}$ Dinarès-Turell, J., and Tinto, K., 2014. Data report: paleomagnetism and rock magnetism of sediments from offshore Canterbury Basin, IODP Expedition 317. In Fulthorpe, C.S., Hoyanagi, K., Blum, P., and the Expedition 317 Scientists, Proc. IODP, 317: Tokyo (Integrated Ocean Drilling Program Management International, Inc.). doi:10.2204/iodp.proc.317.206.2014 2 Istituto Nazionale di Geofisica e Vulcanologia, Via di Vigna Murata 605, I-00143 Rome, Italy. jaume.dinares@invg.it

${ }^{3}$ Department of Geology, University of Otago, PO Box 56, Dunedin 9054, New Zealand. ${ }^{4}$ Current address: Lamont-Doherty Earth Observatory, Columbia University, PO Box 1000, Palisades NY 10964-8000, USA.

\section{Abstract}

Paleomagnetic and rock magnetic analyses were conducted on discrete samples from sediments drilled during Integrated Ocean Drilling Program Expedition 317 in the Canterbury Basin offshore New Zealand to assess the nature of magnetic remanence and to evaluate the directional data for a magnetic reversal stratigraphy. The sediments consist of relatively coarse grained lithologies from shelf to slope settings. The presence of several hiatuses, together with very low core recovery in some intervals as well as drillingrelated magnetic overprints, prevented establishment of a good shipboard magnetostratigraphy. In an effort to overcome some of the magnetic shortcomings and to substantiate shipboard measurements (overprints and partial demagnetization to $20 \mathrm{mT}$ ), natural remanent magnetization and subsequent full stepwise alternating field or thermal demagnetization measurements were taken on 765 individual discrete samples from Holes U1351B (131 samples), U1352B/U1352C (485 samples), U1353B (54 samples), and U1354B/U1354C (95 samples). Some rock magnetic measurements, including thermomagnetic curves, were taken on a few representative samples to acquire information on the magnetic carriers. The remanence intensity of the sediments is relatively low (usually $10^{-3}$ to $10^{-5} \mathrm{~A} / \mathrm{m}$ ) and often displays unstable behavior upon demagnetization. Demagnetization data have been separated into four different classes based on their quality. Classes 1 and 2 are of sufficient quality to be used for magnetostratigraphic purposes and represent $\sim 40 \%$ of the studied samples (although some of them could still represent a steep magnetic drilling overprint). Considering the calculated characteristic remanent magnetization inclination, a number of polarity zones can be defined. However, the fragmentary nature of the data set, mostly related to core recovery in addition to detected and undetected sedimentary hiatuses, makes determination of the precise position of the reversal boundaries and their correlation to the geomagnetic polarity timescale a difficult task. Nevertheless, when combined shipboard and postcruise discrete measurements are placed within biostratigraphic constraints, some magnetostratigraphic insights can potentially refine the available age model. These insights include the identification of the Matuyama/Brunhes boundary (C1r1r/C1n, $0.778 \mathrm{Ma})$ at all four sites, the Gauss/Matuyama boundary (C2An.1n/C2r.2r, 2.581 Ma) in Hole U1351B, and also the position of the Olduvai Subchron (C2n, 1.778-1.945 Ma) in Hole 
U1351B, which relates to the position of the classical Pliocene/Pleistocene boundary.

\section{Introduction}

Integrated Ocean Drilling Program (IODP) Expedition 317 was devoted to understanding the relative importance of global sea level (eustasy) versus local tectonic and sedimentary processes in controlling continental margin sedimentary cycles. Upper Miocene to recent sedimentary sequences were cored in a transect of three sites on the continental shelf (landward to basinward, Sites U1353, U1354, and U1351) and one site on the continental slope (Site U1352) in the Canterbury Basin on the eastern margin of the South Island of New Zealand (see the "Expedition 317 summary" chapter [Expedition 317 Scientists, 2011a]). Obtaining the most accurate age model for the drilled sediments is pivotal for attaining the main goals of the expedition. Shipboard paleomagnetic measurements taken during Expedition 317 had limited success because of a strong, pervasive drilling overprint on the natural remanent magnetization (NRM) because of the use of magnetic core barrels for most of the drilled interval, preventing the unambiguous identification of a characteristic primary component (characteristic remanent magnetization [ChRM]). Shipboard measurements demagnetized the archive section halves to $20 \mathrm{mT}$ but seldom removed the overprint along those intervals. Advanced piston coring with nonmagnetic core barrels produced reasonably good magnetic results. However, these barrels could only be used for the upper $60-70 \mathrm{~m}$ for the shelf sites or to $\sim 250 \mathrm{~m}$ at the slope site where stiffness of the sediments and/or the presence of coarse-grained material prevented further use. Unfortunately, given the relatively high sediment accumulation rate, the upper part of the interval drilled with nonmagnetic barrels usually comprised only the youngest magnetic polarity magnetozone (the Brunhes Chron), precluding retrieving a sensible magnetostratigraphy downhole. Here, we report on a detailed paleomagnetic study for 764 individual discrete samples from Holes U1351B (water depth $122 \mathrm{~m}$; 131 samples), U1352B/U1352C (water depth $354 \mathrm{~m} ; 485$ samples), U1353B (water depth $85 \mathrm{~m}$; 54 samples), and U1354B/U1354C (water depth $113 \mathrm{~m}$; 95 samples) in an attempt to substantiate the nature of the remanence and to evaluate the potential for developing magnetostratigraphic constraints on the chronology of these sediments.

\section{Methods and materials}

Shore-based measurements were taken at the paleomagnetic laboratories of the Istituto Nazionale di Geofisica e Vulcanologia in Rome (Italy) and at Otago University (New Zealand). Each of these laboratories is equipped with a $2 \mathrm{G}$ Enterprises three-axis direct-current superconducting quantum interference device pass-through superconducting rock magnetometer (manufacturer noise level of $10^{-6} \mathrm{~A} / \mathrm{m}$ ) placed in a shielded room and including in-line three-axis degaussing coils for remanence measurement and demagnetization. NRM was measured followed by stepwise alternating field (AF) demagnetization. Samples were measured and demagnetized along the three axes following a protocol that included field strengths progressively increasing in 5 $\mathrm{mT}$ increments from 5 to $100 \mathrm{mT}$ for the Otago measured samples (OT) and an adjusted protocol for the Rome (RM) measured samples $(4,8,13,17,21,25$, $30,35,40,45,50,60,80$, and $100 \mathrm{mT}$ demagnetization). A small subset of samples was thermally demagnetized to $450^{\circ} \mathrm{C}$, but this was restricted to lithified samples from Hole U1352C, which did not have to be contained in plastic cubes. Thermal demagnetization was performed in an ASC oven, located in the low-field laboratory at Otago University, capable of heating specimens up to $800^{\circ} \mathrm{C}$ (thermal measurements at Rome were discarded due to technical faults). Specimens were stepwise heated at $30^{\circ} \mathrm{C}$ intervals for $30 \mathrm{~min}$. Orientation and intensity of magnetization were measured on the $2 \mathrm{G}$ cryogenic magnetometer after each heating step. Magnetic components were calculated on the orthogonal demagnetization diagrams using a least-squares fitting technique, including the maximum angular deviation (MAD) to better assess quality. Data and plots were analyzed using PuffinPlot software (Lurcock and Wilson, 2012). Magnetic susceptibility was also measured at each step using a Bartington loop sensor in-line with the magnetometer. This measurement monitored alteration of magnetic minerals after heating. To determine Curie temperature, magnetic susceptibility was measured as a function of temperature using a Kappabridge magnetic susceptibility meter with a furnace attachment (model MFK1-FA; Agico Ltd.).

On average, one discrete cubic sample with an effective volume of $\sim 7 \mathrm{~cm}^{3}$ was taken aboard the R/V JOIDES Resolution from each section recovered, with the intent of characterizing the magnetic behavior of the 
sediments in shore-based laboratories. The lithology is mainly dark gray to greenish gray or olive-gray and mud and sandy mud with lesser shell hash, sand, and muddy sand. For paleomagnetic purposes, the lithology with the finest grain size was usually targeted. Sampling procedure and conventions are described in the "Methods" chapter (Expedition 317 Scientists, 2011b). A total of 765 individual discrete samples were measured and data are reported below.

\section{Results}

\section{NRM and directional measurements}

Sample NRM intensity and directional data are given in Tables T1, T2, T3, and T4. NRM intensity ranges from $4.40 \times 10^{-6}$ to $8.85 \times 10^{-1} \mathrm{~A} / \mathrm{m}$ with most samples between $10^{-3}$ and $10^{-5} \mathrm{~A} / \mathrm{m}$ (median $=3.17 \times 10^{-4}$ $\mathrm{A} / \mathrm{m}$ ). These intensities are relatively low but measurable with confidence on the shielded $2 \mathrm{G}$ cryogenic magnetometer. The intensity of some samples rapidly decreases to $\sim 10 \%$ or less of the original NRM magnetization during demagnetization at low fields (below $20 \mathrm{mT}$ ), and reliability of measurement and stability at higher fields seems compromised (Fig. F1D). Samples of this kind and others for which erratic behavior upon demagnetization was observed have been ranked the lowest quality in the sample collection (Class 4), and no ChRM has been computed. Other samples display a more progressive decay upon demagnetization, and some of them do not fully demagnetize at the maximum field applied $(100 \mathrm{mT})$. These samples usually demagnetize a steep downward component up to 20-25 $\mathrm{mT}$ and a second component at a higher field that usually trends toward the origin of the diagram that is considered the ChRM (Fig. F1A). Class 1 samples include those of higher quality trajectories that include several steps and usually have MAD angles $<10$ (i.e., Fig. F1A, F2A), whereas for Class 2 samples, the ChRM is less well defined and MAD angles are usually $<20$ (Fig. F1B). Class 3 samples include those for which a ChRM can be computed (usually not including consecutive steps), but they are classified not reliable and were not considered for the magnetostratigraphic determinations. Some samples notably increase in intensity above $60 \mathrm{mT}$ (Fig. F2C) and define a horizontal direction along the $x-y$ axis of the samples. This is the result of acquisition of a gyroremanence (a magnetic remanence acquired by magnetically anisotropic materials that are rotated within an alternating magnetic field) related to the static application of the three-orthogonal AF demagnetization, but the ChRM component can still be calculated in the range of 20-60 mT. This type of behavior has been described before and appears com- monly associated with iron sulfides such as greigite (e.g., Fu et al., 2008). The presence of magnetic iron sulfides in the studied sediments can also be inferred from the thermal demagnetization of some samples (Fig. F3H), in which a typical decrease in intensity is observed around $360^{\circ} \mathrm{C}$. However, most samples do not show the gyroremanence acquisition, and it is likely that the magnetic carriers are a combination of (titano)magnetites and iron sulfides (see below). It should be noted that dissolution of magnetic minerals and remagnetization of continental shelf sediments have been described in the literature (e.g., Oda and Torii, 2004; Rowan and Roberts, 2006).

The ChRM inclination for the 305 Class 1 and 2 samples form two populations with negative (normal) and positive (reversed) inclinations (Fig. F4) with average values of about $-50^{\circ} / 50^{\circ}$, respectively, that are shallower than the expected geocentric axial dipole values for the site latitude. It is common for marine sediments to acquire a shallower magnetization related to depositional processes or compaction (Arason and Levi, 1990), and this may indicate the successful removal of the steep overprint for most of the Class 1 and 2 samples. However, the ChRM component of samples from the deep part of Hole U1352C below $\sim 1600 \mathrm{~m}$ core depth below seafloor, Method-A (CSF-A) displays a single steep downward component (e.g., Fig. F3I), indicating unsuccessful removal of the pervasive drilling overprint in those cases.

\section{Thermomagnetic measurements}

In addition to the shipboard measurement of isothermal remanent magnetization (IRM), IRM acquisition curves, and subsequent AF demagnetization (see individual site chapters), variations of low-field magnetic susceptibility versus temperature (thermomagnetic curves) were also measured. Figure F4 illustrates typical examples of thermomagnetic curves. For some samples, two successive heating/cooling runs to higher maximum temperatures were measured (Fig. F5A, F5B, F5F) to evaluate reversibility and the eventual formation of new mineral phases upon heating. In some instances heating to $400^{\circ} \mathrm{C}$ results in no reversibility (Fig. F5A), suggesting that a new magnetic mineral phase has formed. The new phase appears to be magnetite, as seen from Curie temperatures of $580^{\circ} \mathrm{C}$ on the successive heating run up to $700^{\circ} \mathrm{C}$. Heating to $380^{\circ} \mathrm{C}$ produces mostly reversible curves in some samples (Fig. F5B), although magnetite with a Curie temperature of $\sim 580^{\circ} \mathrm{C}$ can again be observed on the successive heating run. Therefore, the presence of magnetite in the original samples remains ambiguous. However, for some samples, lower Curie temperatures around $500^{\circ} \mathrm{C}$ have been measured (Fig. F5E), suggesting the pres- 
ence of some sort of titanomagnetite. No direct evidence of magnetic iron sulfides has been observed in the thermomagnetic curves, although they appear to be ubiquitous in some intervals as deduced from NRM thermal demagnetization and inferences from gyroremanence acquisition. Collectively, all the experiments suggest the presence of a combination of magnetic iron sulfides (possibly greigite) and (titano)magnetite in the shelf/slope sediments of the Canterbury Basin.

\section{Magnetic stratigraphy}

\section{Hole U1351B}

Demagnetization data are found in Table T1. NRM intensity and ChRM inclination for discrete samples from the upper $270 \mathrm{~m}$ of Hole U1351B are plotted in Figure F6 along with shipboard data (see the "Site U1351" chapter [Expedition 317 Scientists, 2011c]). Despite the fragmentary nature of the record due to low core recovery, some magnetostratigraphic constraints emerge. Only Class 1 and 2 samples are used for magnetozone polarity determinations in Figure F6. Magnetic polarity appears to be mostly normal (negative inclination) to Core 317-U1351B-9H at $\sim 63 \mathrm{~m}$ CSF-A, with the exception of three relatively thin intervals where single Class 2 reversed polarity (positive inclination) intervals exist (Fig. F1B). The normal interval can be assigned to the Brunhes Chron $(\mathrm{C} 1 \mathrm{n})$, whereas the thin reversed intervals could either represent short excursions within the Brunhes Chron or some sort of secondary component or remagnetization (we note a noisy signal in shipboard measurements within this interval). Core $11 \mathrm{H}$ contains Class 2 reversed polarity samples, thus loosely defining a reversed to normal boundary (R/N_1) between Sections 9H-6 and $11 \mathrm{H}-1$ that must represent the Matuyama/Brunhes transition, even though part of Brunhes and/or Matuyama may be missing. Below Core $11 \mathrm{H}$, recovery is very fragmentary, but all samples measured down to Core $18 \mathrm{X}$ appear to be reversed polarity (i.e., Fig. F2B, F2C). Core 19X includes high-quality samples of normal polarity (negative inclination) (e.g., Fig. F2D), whereas Cores 20X and 21X include Class 1 samples with reversed polarity. Consequently, the N/R_1 and R/N_2 polarity transitions can be constrained (Fig. F6). Another pair of reversal boundaries can be placed farther down in the hole (N/R_2 and R/N_3). These reversal boundaries are shown in Figure F7, which summarizes shipboard biostratigraphic constraints on an age-depth plot (see the "Expedition 317 summary" chapter [Expedition 317 Scientists, 2011a]) correlated to the geomagnetic polarity timescale (Lourens et al., 2004; Ogg et al., 2008). Consequently, the N/R_1 reversal may be correlated to the top of the
Olduvai Subchron (C2n/C1r.3r, $1.778 \mathrm{Ma})$ and the RN_2 reversal to the Chron C2r.1r/C2n boundary (1.945 Ma) (Fig. F7). It is important to note that Surface U1351B-S6 (see the "Expedition 317 summary" chapter [Expedition 317 Scientists, 2011a]) is a sharp basal contact at Section 19X-2, $104 \mathrm{~cm}$ $(144.14 \mathrm{~m})$, between a $1.15 \mathrm{~m}$ thick shelly sand bed and an underlying mud with scattered shell fragments. A short normal polarity interval occurs in Sections 19X-3 and 19X-4 (Table T1), and therefore the upper part of the normal chron (C2n, Olduvai) and also the lower part of the reversed chron (C1r.3r) could have been truncated. Alternative reasoning without violating the biostratigraphic constraints could also correlate the normal chron in Core 19X to the older Reunion Subchron (C2r.1n, 2.128-2.148 Ma) (open stars in Fig. F7). Integrating additional biostratigraphic data could lead to refined correlation around this interval that relates to the classic Pliocene/Pleistocene boundary. Note that arguments to retain the base of the Quaternary system coincident with the base of the Calabrian stage of the Pleistocene series (1.806 Ma) failed when in 2009 the International Commission on Stratigraphy redefined the base of the Quaternary coincident with the base of the Gelasian stage (2.58 Ma), formerly the uppermost stage of the Pliocene. Finally, the N/R_2 reversal seems to correlate unambiguously with the top of Chron C2An.1n (2.581 Ma), whereas R/N_3 is best correlated to the base of Chron C2An.3n (the Gilbert/Gauss Chron boundary at 3.596 Ma).

\section{Holes U1352B and U1352C}

Demagnetization data are listed in Table T2. NRM intensity and ChRM inclination for discrete samples from Holes U1352B and U1352C are plotted in Figures F8 and F9, respectively. The upper $240 \mathrm{~m}$ of Hole U1352B consists of normal samples (e.g., Fig. F2A), with the exception of one or two intervals with samples that clearly depict a reversed ChRM component (e.g., Fig. F3B). Shipboard data suggested reversed polarities at Core 317-U1352B-28H and below, but because at this level magnetic core barrels were introduced and because of the limited single 20 mT demagnetization step, ambiguity remains as to whether the reversal is meaningful (see the "Site U1352" chapter [Expedition 317 Scientists, 2011d]). Demagnetization of discrete samples from Core $29 \mathrm{H}$ (data from Core $28 \mathrm{H}$ look unreliable) clearly depicts reversed polarities (Fig. F3C), and hence a polarity reversal exists that must correlate to the Brunhes/ Matuyama transition. Further downhole some Class 1 and 2 samples do exist with either normal or reversed ChRM, but the succession does not seem to clearly outline reasonable magnetozones because 
samples with different polarities succeed each other rapidly along the interval from 300 to $500 \mathrm{~m}$ CSF-A, delimiting an interval with zones depicted as ambiguous polarity. All samples measured below $520 \mathrm{~m}$ CSF-A from Hole U1352B contain a negative (normal) ChRM direction. In Hole U1353C, there seems to be a poorly constrained N/R reversal at $\sim 705 \mathrm{~m}$ CSF-A with only one discrete Class 1 reversed sample in Section 317-U1352C-10R-2 (Fig. F9). Downhole, the succession of normal and reversed samples does not allow definition of a clear picture of magnetic zonation. The interval below $1500 \mathrm{~m}$ CSF-A to the Marshall Paraconformity) displays high-quality demagnetization data (i.e., Fig. F3I) with relatively steep downward inclinations and a single component of magnetization that suggests that a pervasive drilling overprint has not been removed.

\section{Shelf Holes U1353B, U1354B, and U1354C}

Demagnetization data are reported in Tables $\mathrm{T} 3$ and T4. NRM intensity and ChRM inclination data for Holes U1353B, U1354B, and U1354C are plotted in Figures F10, F11, and F12, respectively. Holes U1353B and U1354B both present normal polarities down to $\sim 64-65 \mathrm{~m}$ CSF-A. The polarity change in shipboard data is clear for Hole U1354B (Fig. F11) (see the "Site U1354" chapter [Expedition 317 Scientists, 2011e]). Discrete sample data confirm the reversed polarities in Section 317-U1354B-13H-2, whereas only a Class 3 normal sample was measured from Core $12 \mathrm{H}$. Collectively, the Matuyama/Brunhes transition can be established at $64.75 \mathrm{~m}$ CSF-A, although a lithologic boundary at this level suggests a sedimentary hiatus and a possibility that the upper part of the Matuyama Chron (and possibly the lower part of the Brunhes Chron) is missing, as indicated by biostratigraphic constraints (see the "Site U1354" chapter [Expedition 317 Scientists, 2011e]). The Matuyama/Brunhes transition is less obvious in Hole U1353B but has to be placed below Core 317U1353B-10H, for which normal Class 1 and 2 samples were measured. Hole U1354C displays reversed polarity from 66 to $\sim 110$ m CSF-A. Below that depth some normal samples were measured. However, these normal samples are intercalated with goodquality reversed samples within short intervals (Fig. F12), and the pattern does not permit straightforward correlation. Data below 180 m CSF-A become too fragmentary and it was not possible to recover good magnetostratigraphy below that depth.

\section{Acknowledgments}

The samples and data for this research were provided by the Integrated Ocean Drilling Program (IODP).
IODP is sponsored by the U.S. National Science Foundation and participating countries under management of the Consortium for Ocean Leadership. Samples were collected during the cruise and all shipboard scientists, IODP technicians, and the Captain and crew of the JOIDES Resolution are thanked for their help during Expedition 317. We also thank an anonymous reviewer for insightful comments.

\section{References}

Arason, P., and Levi, S., 1990. Models of inclination shallowing during sediment compaction. J. Geophys. Res.: Solid Earth, 95(B4):4481-4499. doi:10.1029/ JB095iB04p04481

Expedition 317 Scientists, 2011a. Expedition 317 summary. In Fulthorpe, C.S., Hoyanagi, K., Blum, P., and the Expedition 317 Scientists, Proc. IODP, 317: Tokyo (Integrated Ocean Drilling Program Management International, Inc.). doi:10.2204/iodp.proc.317.101.2011

Expedition 317 Scientists, 2011b. Methods. In Fulthorpe, C.S., Hoyanagi, K., Blum, P., and the Expedition 317 Scientists, Proc. IODP, 317: Tokyo (Integrated Ocean Drilling Program Management International, Inc.). doi:10.2204/iodp.proc.317.102.2011

Expedition 317 Scientists, 2011c. Site U1351. In Fulthorpe, C.S., Hoyanagi, K., Blum, P., and the Expedition 317 Scientists, Proc. IODP, 317: Tokyo (Integrated Ocean Drilling Program Management International, Inc.). doi:10.2204/iodp.proc.317.103.2011

Expedition 317 Scientists, 2011d. Site U1352. In Fulthorpe, C.S., Hoyanagi, K., Blum, P., and the Expedition 317 Scientists, Proc. IODP, 317: Tokyo (Integrated Ocean Drilling Program Management International, Inc.). doi:10.2204/iodp.proc.317.104.2011

Expedition 317 Scientists, 2011e. Site U1354. In Fulthorpe, C.S., Hoyanagi, K., Blum, P., and the Expedition 317 Scientists, Proc. IODP, 317: Tokyo (Integrated Ocean Drilling Program Management International, Inc.). doi:10.2204/iodp.proc.317.106.2011

Fu, Y., von Dobeneck, T., Franke, C., Heslop, D., and Kasten, S., 2008. Rock magnetic identification and geochemical process models of greigite formation in Quaternary marine sediments from the Gulf of Mexico (IODP Hole U1319A). Earth Planet. Sci. Lett., 275(34):233-245. doi:10.1016/j.epsl.2008.07.034

Lourens, L.J., Hilgen, F.J., Laskar, J., Shackleton, N.J., and Wilson, D., 2004. The Neogene period. In Gradstein, F.M., Ogg, J., et al. (Eds.), A Geologic Time Scale 2004: Cambridge (Cambridge Univ. Press), 409-440.

Lurcock, P.C., and Wilson, G.S., 2012. PuffinPlot: a versatile, user-friendly program for paleomagnetic analysis. Geochem., Geophys., Geosyst., 13(6):Q06Z45. doi:10.1029/2012GC004098

Oda, H., and Torii, M., 2004. Sea-level change and remagnetization of continental shelf sediments off New Jersey (ODP 174A): magnetite and greigite diagenesis. Geophys. J. Int., 156(3):443-458. doi:10.1111/j.1365246X.2004.02162.X 
Ogg, J.G., Ogg, G., and Gradstein, F.M., 2008. The Concise Geologic Time Scale: Cambridge (Cambridge Univ. Press). http://www.cambridge.org/catalogue/catalogue.asp?isbn=9780521898492

Rowan, C.J., and Roberts, A.P., 2006. Magnetite dissolution, diachronous greigite formation, and secondary magnetizations from pyrite oxidation: unravelling complex magnetizations in Neogene marine sediments from
New Zealand. Earth Planet. Sci. Lett., 241(1-2):119-137. doi:10.1016/j.epsl.2005.10.017

Initial receipt: 24 October 2012

Acceptance: 16 January 2014

Publication: 7 May 2014

MS 317-206 
Figure F1. Alternating field (AF) demagnetization behavior illustrating the defined quality/behavior classes for samples from Hole U1351B. A. Class 1. B. Class 2. C. Class 3. D. Class 4. For orthogonal vector plots: open symbols represent data projected on the vertical plane, solid symbols represent data projected on the horizontal plane, red symbols indicate data that are selected for principal component analysis (PCA), and blue line indicates the PCA fit. In equal-area stereo plots: open symbols represent data on the upper hemisphere (negative inclinations), solid symbols represent data on the lower hemisphere (positive inclinations).
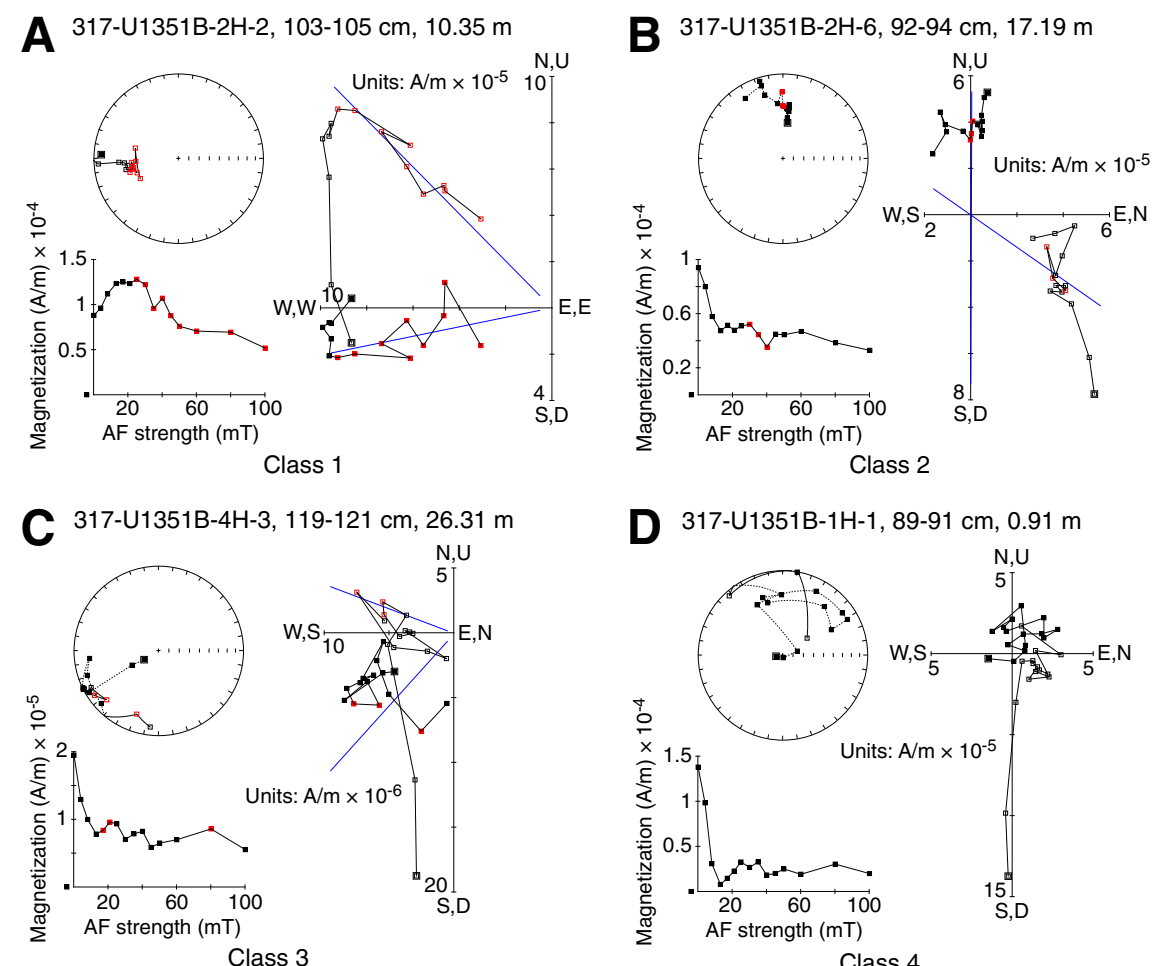
Figure F2. Alternating field (AF) demagnetization behavior for representative normal $(\mathbf{A}, \mathbf{D}, \mathbf{G})$ and reverse (B, C, E, F, H) Class 1 samples, Hole U1351B. See Figure F1 for symbol definitions.

A 317-U1351B-9H-3, 99-101 cm, $61.21 \mathrm{~m}$

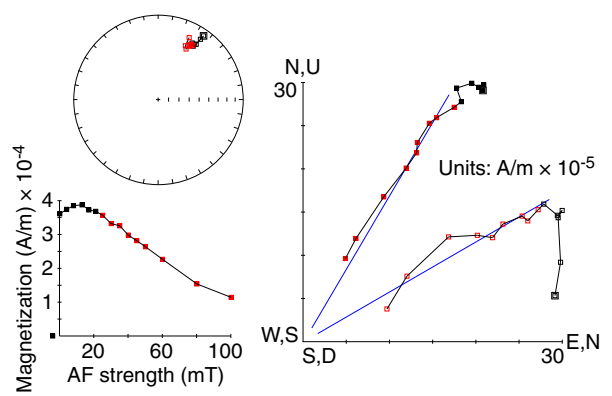

C $317-U 1351 \mathrm{~B}-14 \mathrm{X}-2,24-26 \mathrm{~cm}, 96.46 \mathrm{~m}$

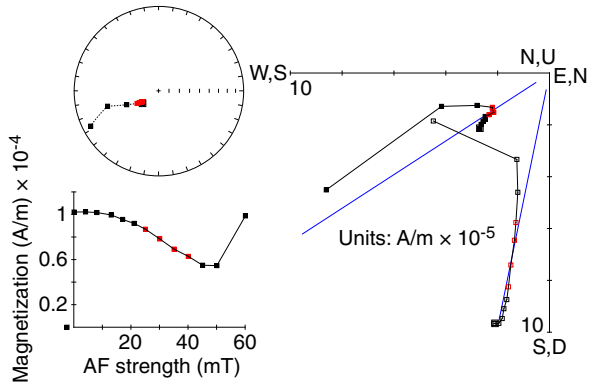

E $317-U 1351 B-20 X-5,70-72 \mathrm{~cm}, 157.92 \mathrm{~m}$

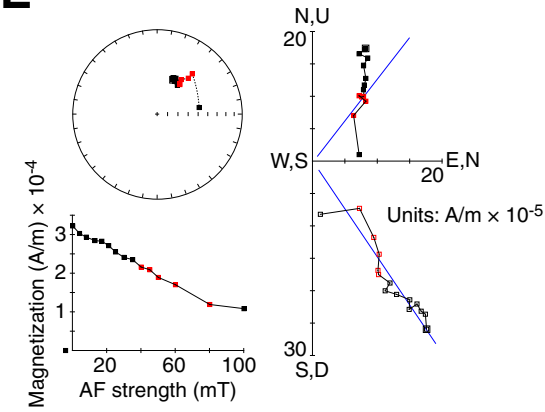

G $317-U 1351 B-29 X-1,85-87 \mathrm{~cm}, 238.37 \mathrm{~m}$

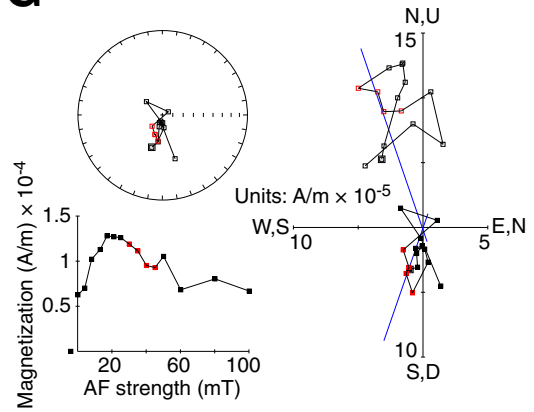

B 317-U1351B-13H-2, 17-19 cm, $86.37 \mathrm{~m}$

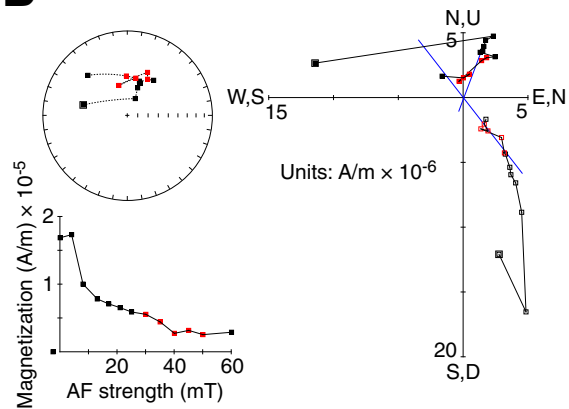

D $317-U 1351 B-19 X-4,69-71 \mathrm{~cm}, 146.81 \mathrm{~m}$

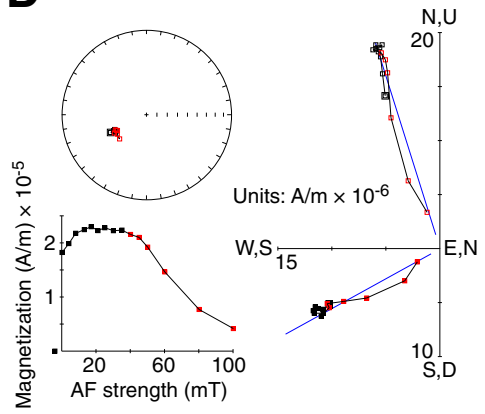

E $317-U 1351 B-27 X-3,87-89 \mathrm{~cm}, 222.29 \mathrm{~m}$

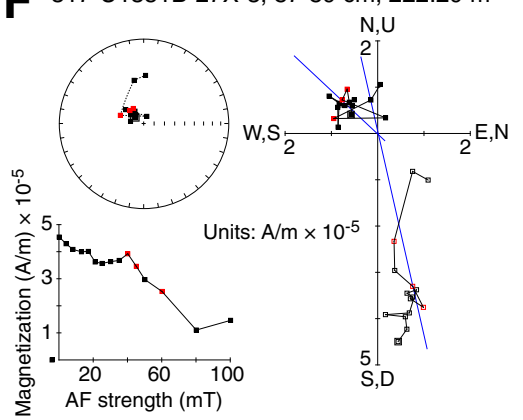

H $317-U 1351 B-31 X-3,103-105 \mathrm{~cm}, 260.75 \mathrm{~m}$

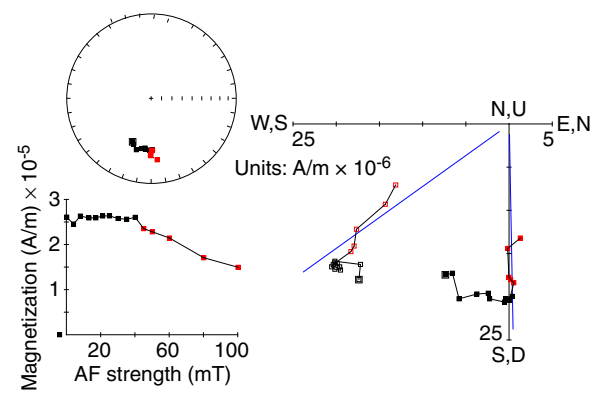


Figure F3. Alternating field (AF) and thermal demagnetization behavior for representative samples from Holes U1352B, U1352C, U1354B, and U1354C. A, D, G, H, J, L. Class 1 samples with normal ChRM component. B, C, E, F, I, K. Class 1 samples with reversed ChRM component. Mag. sus. = magnetic susceptibility. See Figure F1 for symbol definitions.

A $317-U 1352 B-5 H-2,14-16 \mathrm{~cm}, 37.08 \mathrm{~m}$
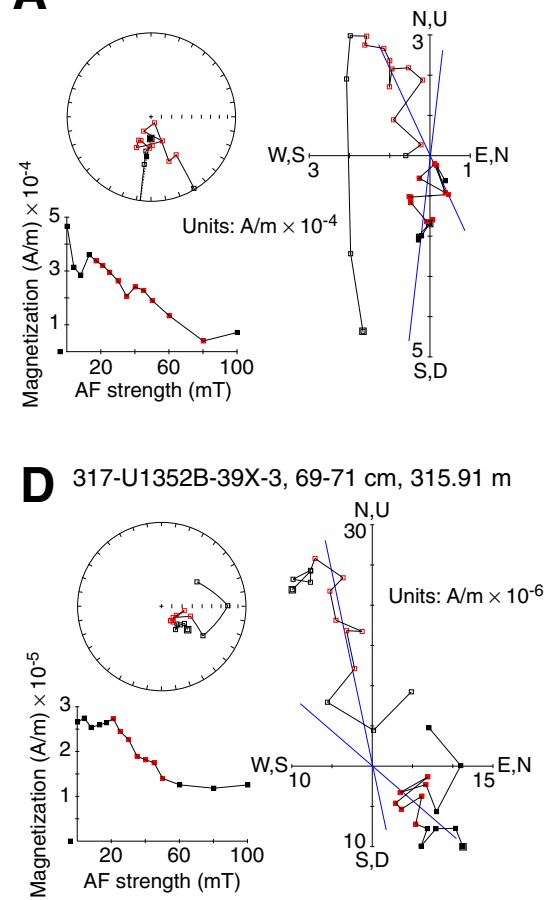

G

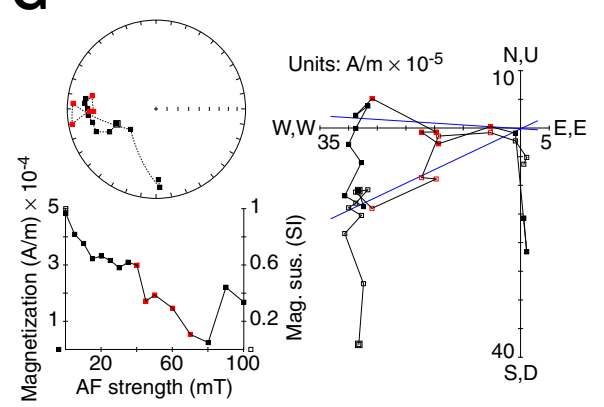

J $317-U 1354 \mathrm{~B}-11 \mathrm{H}-2,121-123 \mathrm{~cm}, 53.23 \mathrm{~m}$
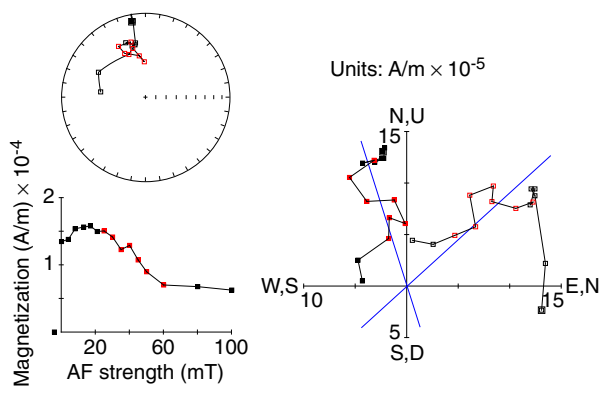

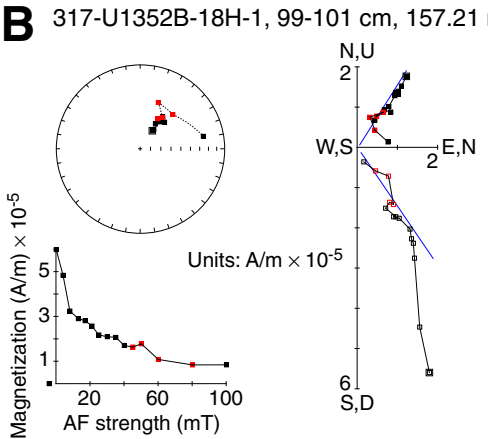

E ${ }^{317-U 1352 B-50 X-4,50-52 ~ c m, ~} 422.92 \mathrm{~m}$

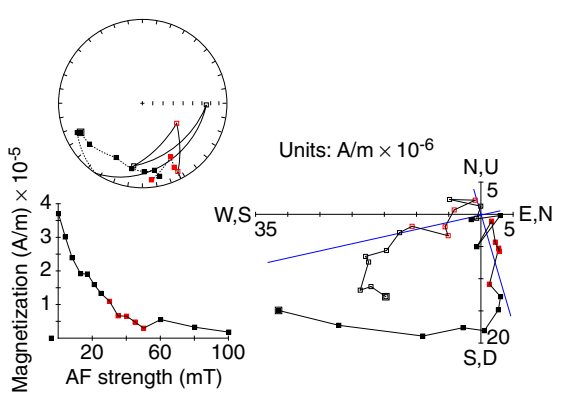

H

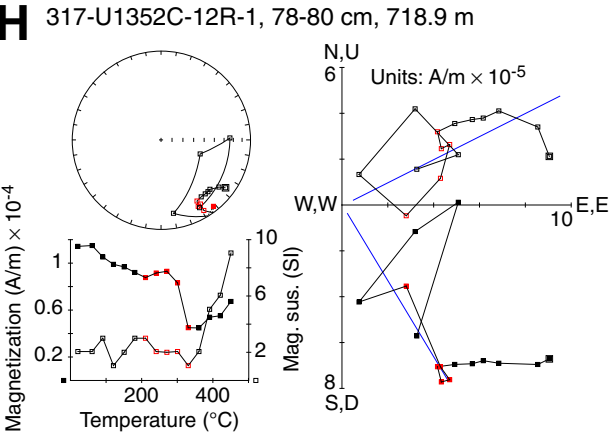

C $317-U 1352 B-31 X-2,35-37 \mathrm{~cm}, 269.07 \mathrm{~m}$

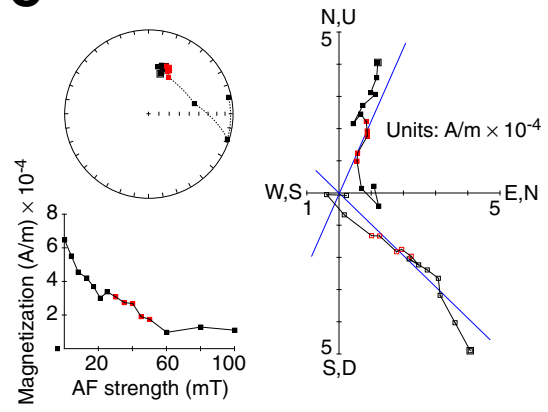

F $317-U 1352 B-73 X-1,106-108 \mathrm{~cm}, 630.18 \mathrm{~m}$

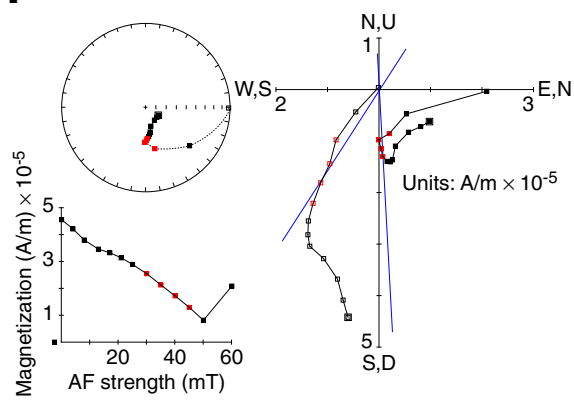

317-U1352C-119R-6, 110-112 cm, $1670.1 \mathrm{~m}$

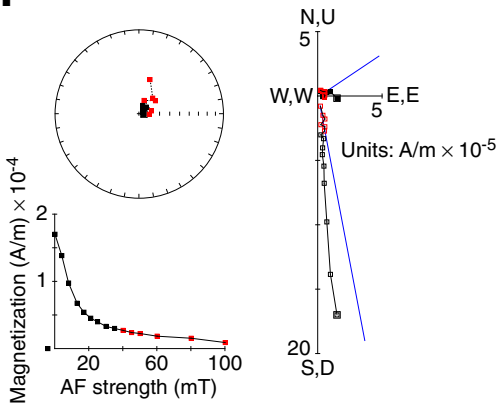

K 317-U1354B-13H-5, 73-75 cm, 69.25 m

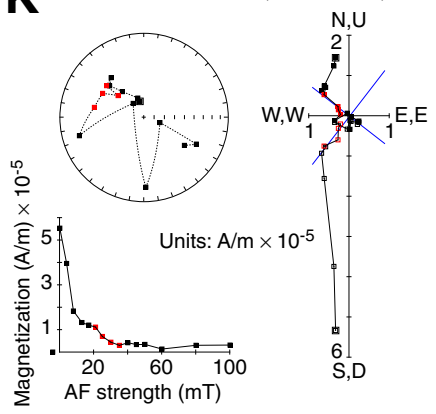

317-U1354C-10X-4, 33-35 cm, $130.45 \mathrm{~m}$

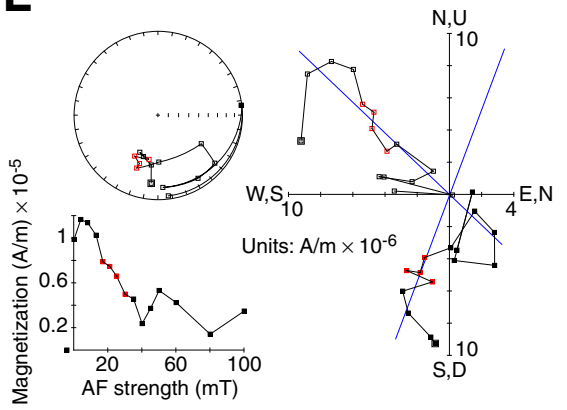


Figure F4. Histogram of Class 1 and 2 normal and reversed polarity ChRM inclinations for discrete samples, Expedition 317. Vertical lines indicate inclinations for a geocentric axial dipole (GAD) field at the site latitude.

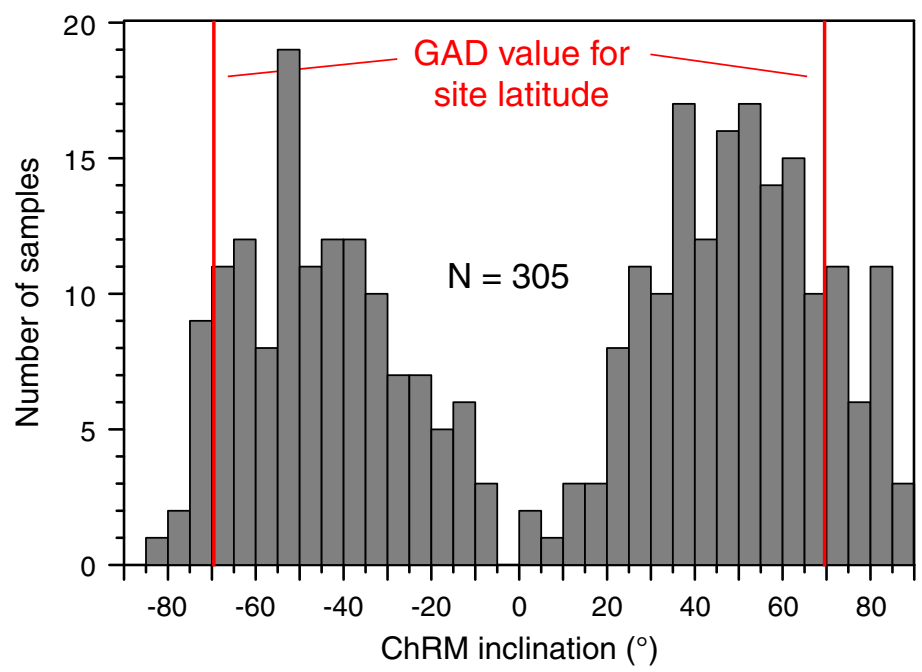


Figure F5. A-F. Representative thermomagnetic curves (red = heating, blue = cooling) for Expedition 317 sediment.
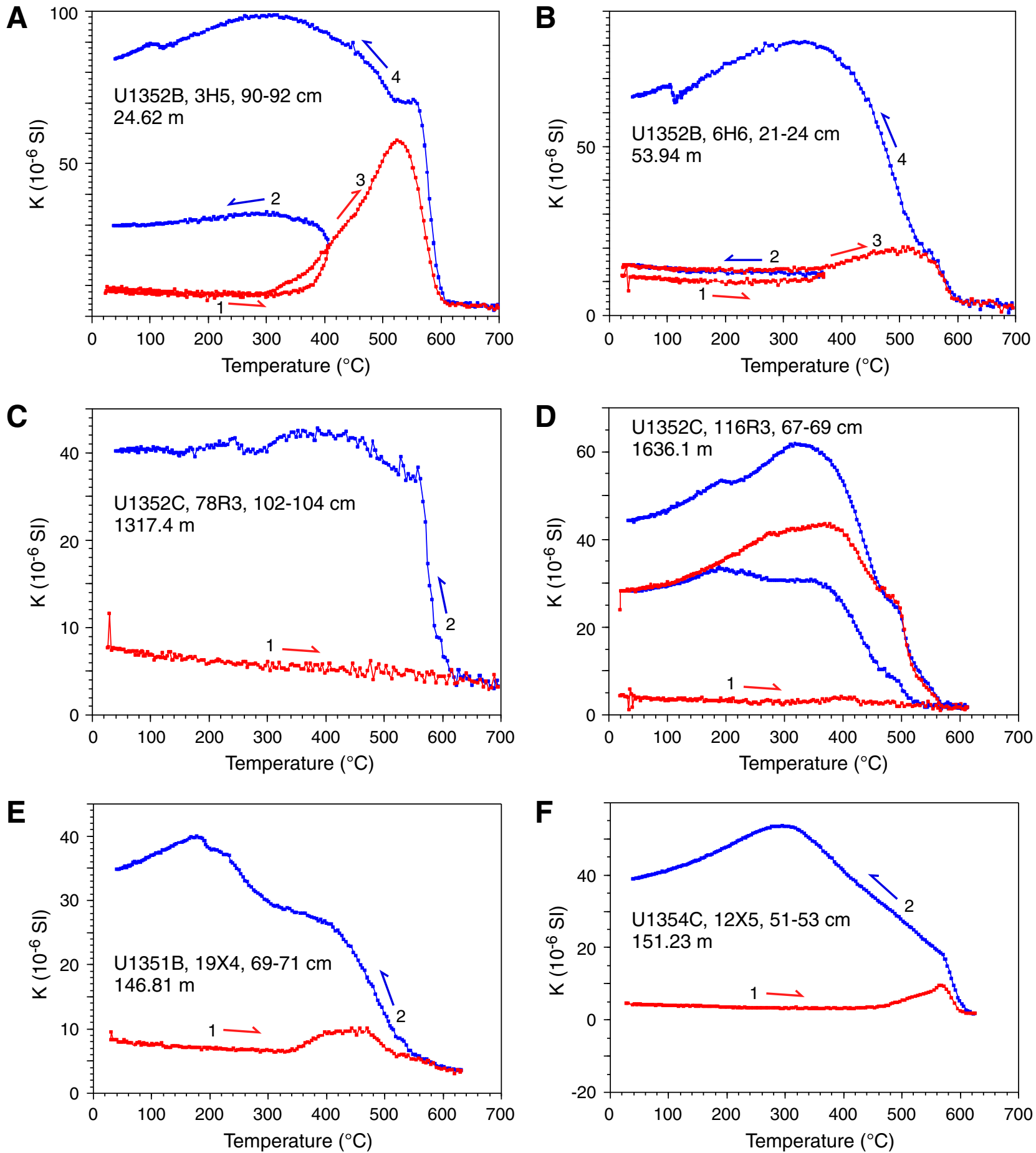
Figure F6. Natural remanenent magnetization (NRM) intensity and characteristic remanent magnetization (ChRM) inclination for discrete samples from the upper $270 \mathrm{~m}$ of Hole U1351B. Shipboard section-half NRM intensities and ChRM inclinations after $20 \mathrm{mT}$ AF demagnetization are also plotted. The quality of the demagnetization data on the discrete samples has been ranked in four different classes (Class $1=$ higher quality, Class $4=$ no interpretation possible; see text for explanation). Polarity indicates the inferred ChRM polarity based only on Class 1 and 2 samples (normal polarity for negative inclination and reversed polarity for positive inclination). Four recognized reversed to normal $(\mathrm{R} / \mathrm{N})$ or normal to reversed $(\mathrm{N} / \mathrm{R})$ magnetochron boundaries are labeled from top to base.

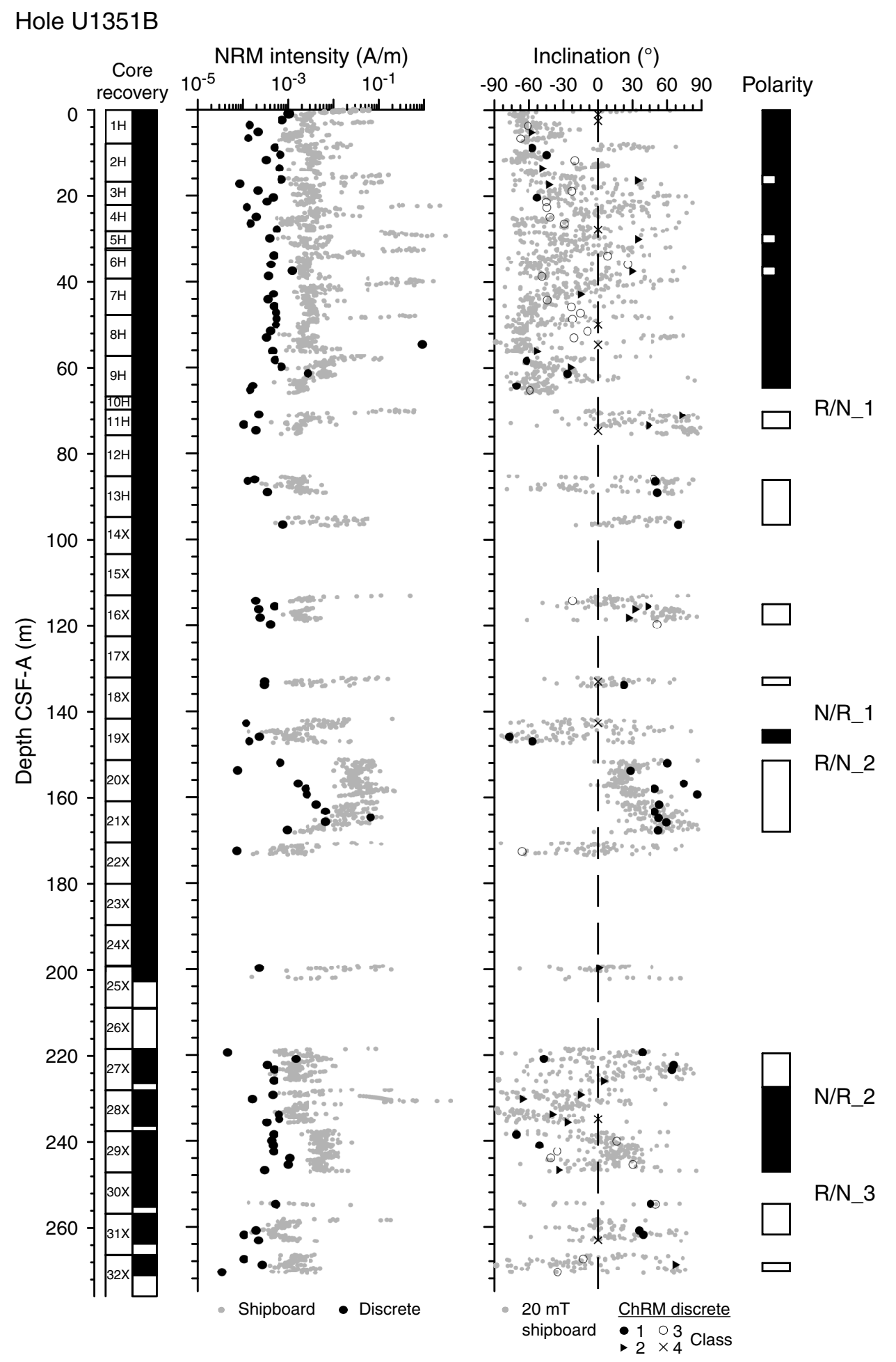


Figure F7. Age-depth plot for the upper 270 m encompassing lithologic Unit I of Hole U1351B that integrates biostratigraphic ages and inferred magnetostratigraphic constraints. Hiatuses are inferred from biostratigraphic analysis. Hiatus positions are sometimes located at distinct boundaries found in recovered cores, but some hiatuses exist in intervals of low recovery. Dashed wavy lines $=$ the most poorly constrained hiatuses. NZ $=\mathrm{New}$ Zealand. Nann. = nannofossil, Plankt. = planktonic.

Hole U1351B

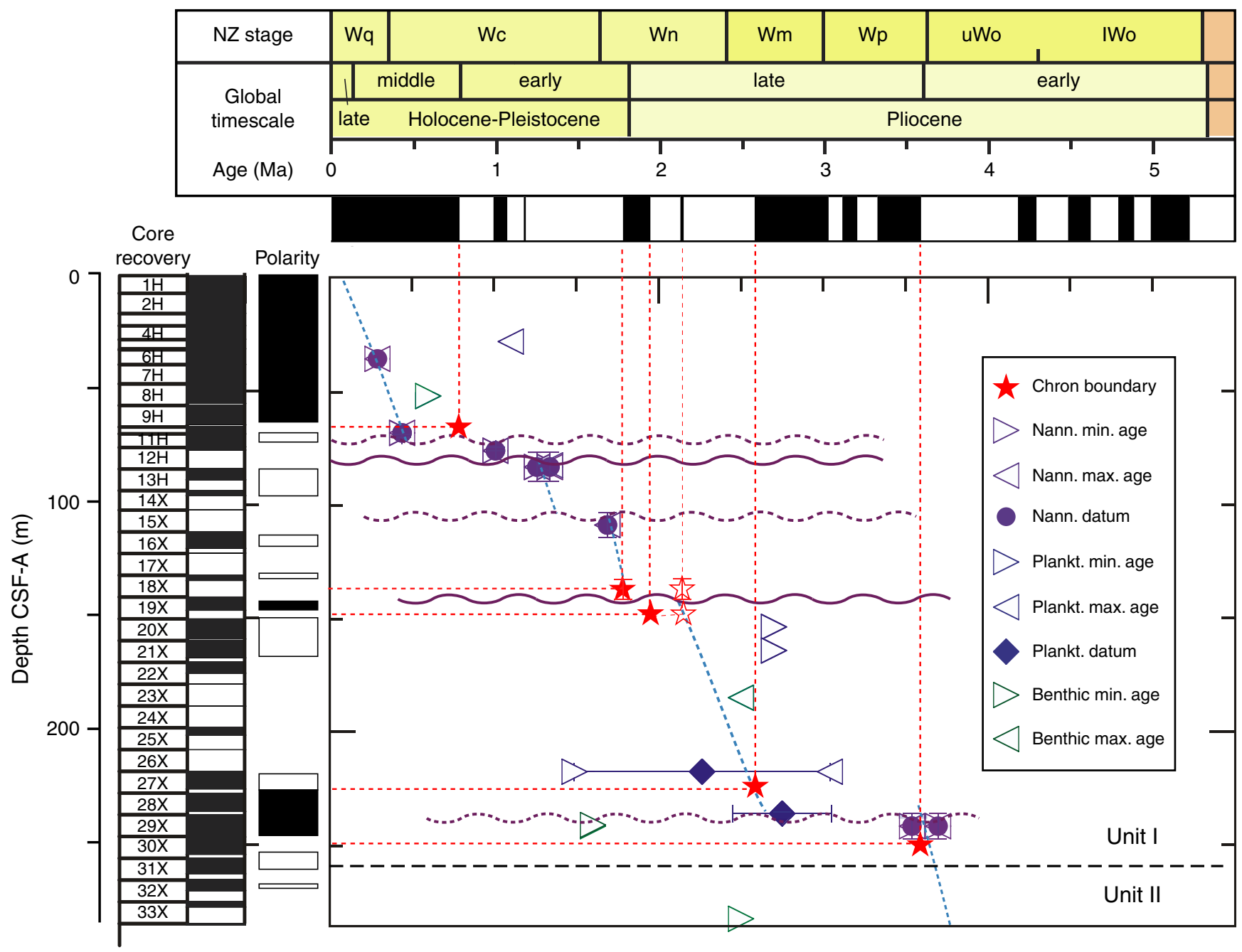


Figure F8. Natural remanenent magnetization (NRM) intensity and characteristic remanent magnetization (ChRM) inclination for discrete samples from Hole U1352B. Shipboard section-half NRM intensities and ChRM inclinations after $20 \mathrm{mT}$ AF demagnetization are also plotted. The quality of the demagnetization data on the discrete samples has been ranked in four different classes (Class $1=$ higher quality, Class $4=$ no interpretation possible; see text for explanation). Polarity indicates the inferred ChRM polarity based only on Class 1 and 2 samples (normal polarity for negative inclination and reverse polarity for positive inclination). APC $=$ advanced piston corer, $\mathrm{XCB}=$ extended core barrel.

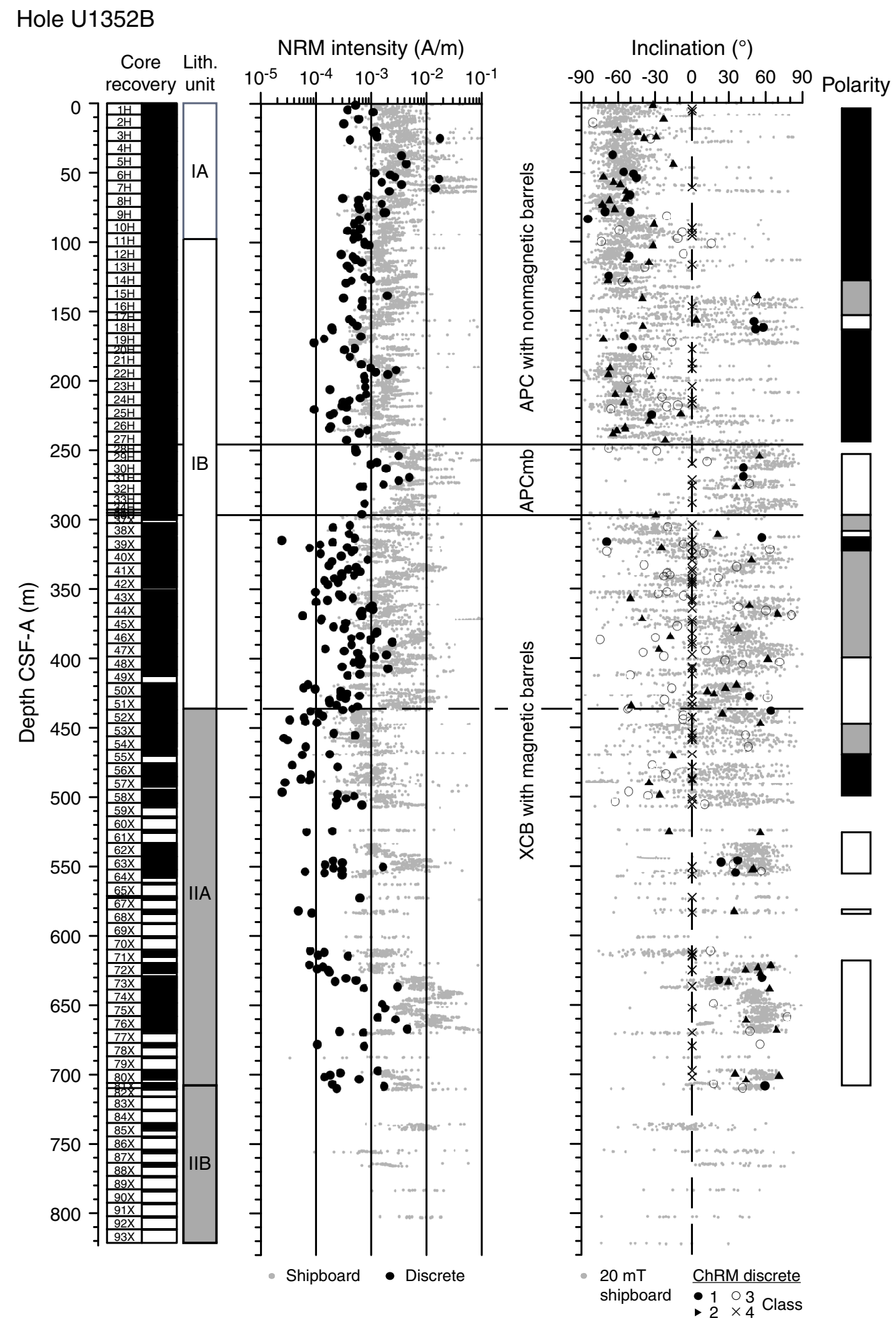


Figure F9. Natural remanenent magnetization (NRM) intensity and characteristic remanent magnetization (ChRM) inclination for discrete samples from Hole U1352C. Shipboard section-half NRM intensities and ChRM inclinations after $20 \mathrm{mT}$ AF demagnetization are also plotted. The quality of the demagnetization data on the discrete samples has been ranked in four different classes (Class $1=$ higher quality, Class $4=$ no interpretation possible; see text for explanation). Polarity indicates the inferred ChRM polarity based only on Class 1 and 2 samples (normal polarity for negative inclination and reverse polarity for positive inclination). ?? = uncertain polarity.

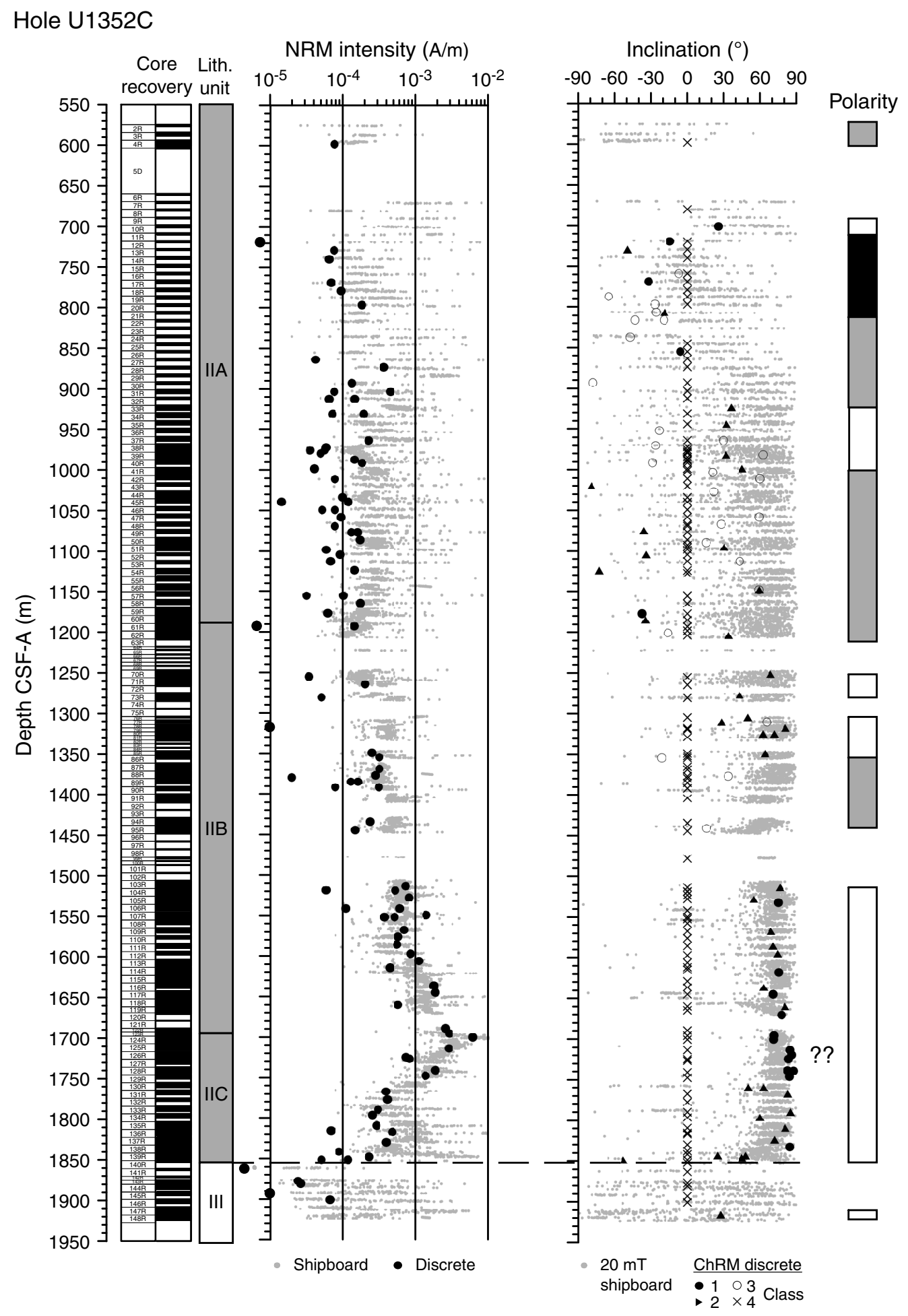


Figure F10. Natural remanent magnetization (NRM) intensity and characteristic remanent magnetization (ChRM) inclination for discrete samples from the upper $220 \mathrm{~m}$ of Hole U1353B. Shipboard section-half NRM intensities and ChRM inclinations after $20 \mathrm{mT}$ AF demagnetization are also plotted. The quality of the demagnetization data on the discrete samples has been ranked in four different classes (Class $1=$ higher quality, Class $4=$ no interpretation possible; see text for explanation). Polarity indicates the inferred ChRM polarity based only on Class 1 and 2 samples (normal polarity for negative inclination and reverse polarity for positive inclination). $\mathrm{APC}=$ advanced piston corer, $\mathrm{XCB}=$ extended core barrel.

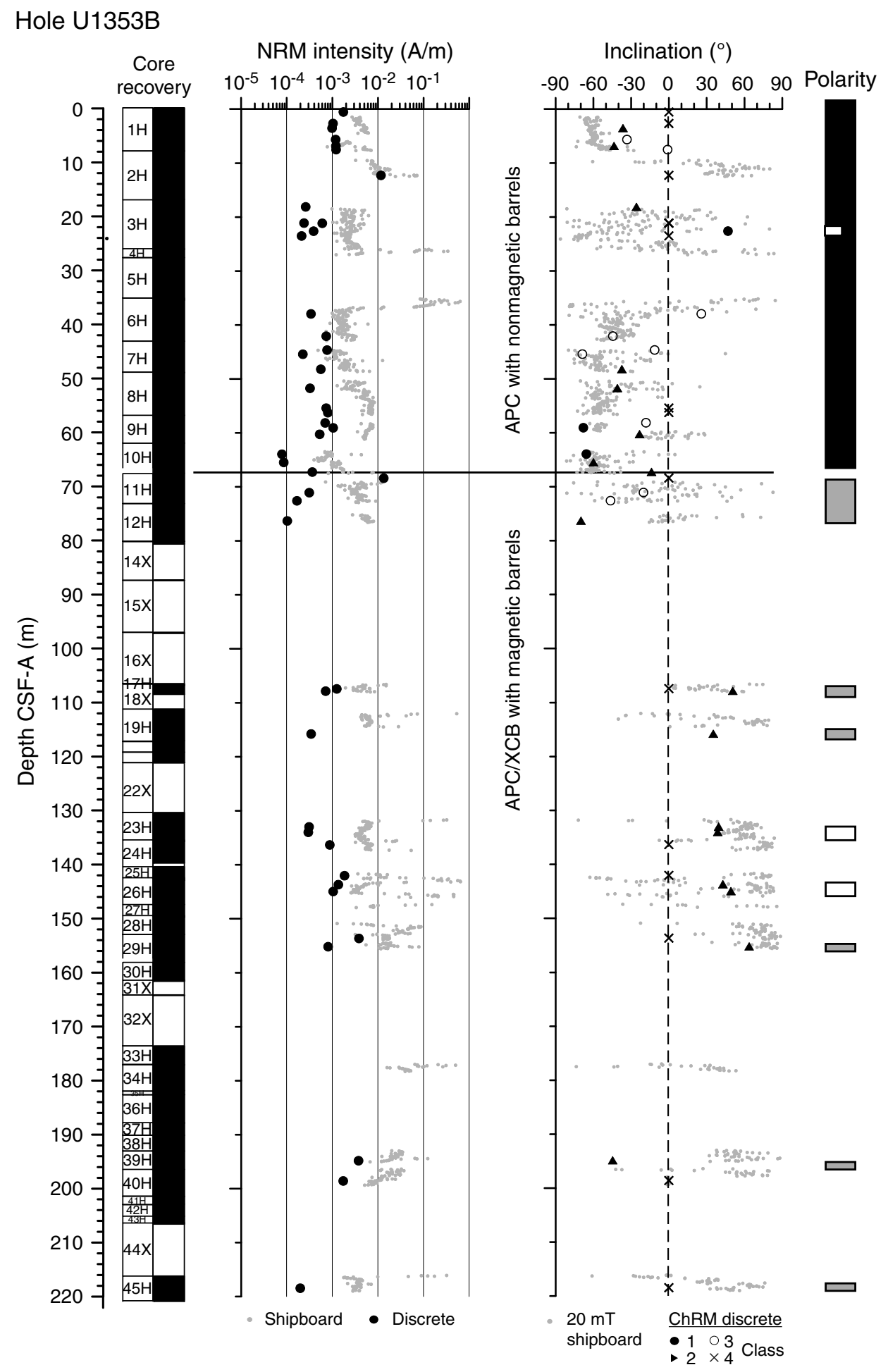


Figure F11. Natural remanenent magnetization (NRM) intensity and characteristic remanent magnetization (ChRM) inclination for discrete samples from Hole U1354B. Shipboard section-half NRM intensities and ChRM inclinations after $20 \mathrm{mT}$ AF demagnetization are also plotted. The quality of the demagnetization data on the discrete samples has been ranked in four different classes (Class $1=$ higher quality, Class $4=$ no interpretation possible; see text for explanation). Polarity indicates the inferred ChRM polarity based only on Class 1 and 2 samples (normal polarity for negative inclination and reverse polarity for positive inclination).

Hole U1354B

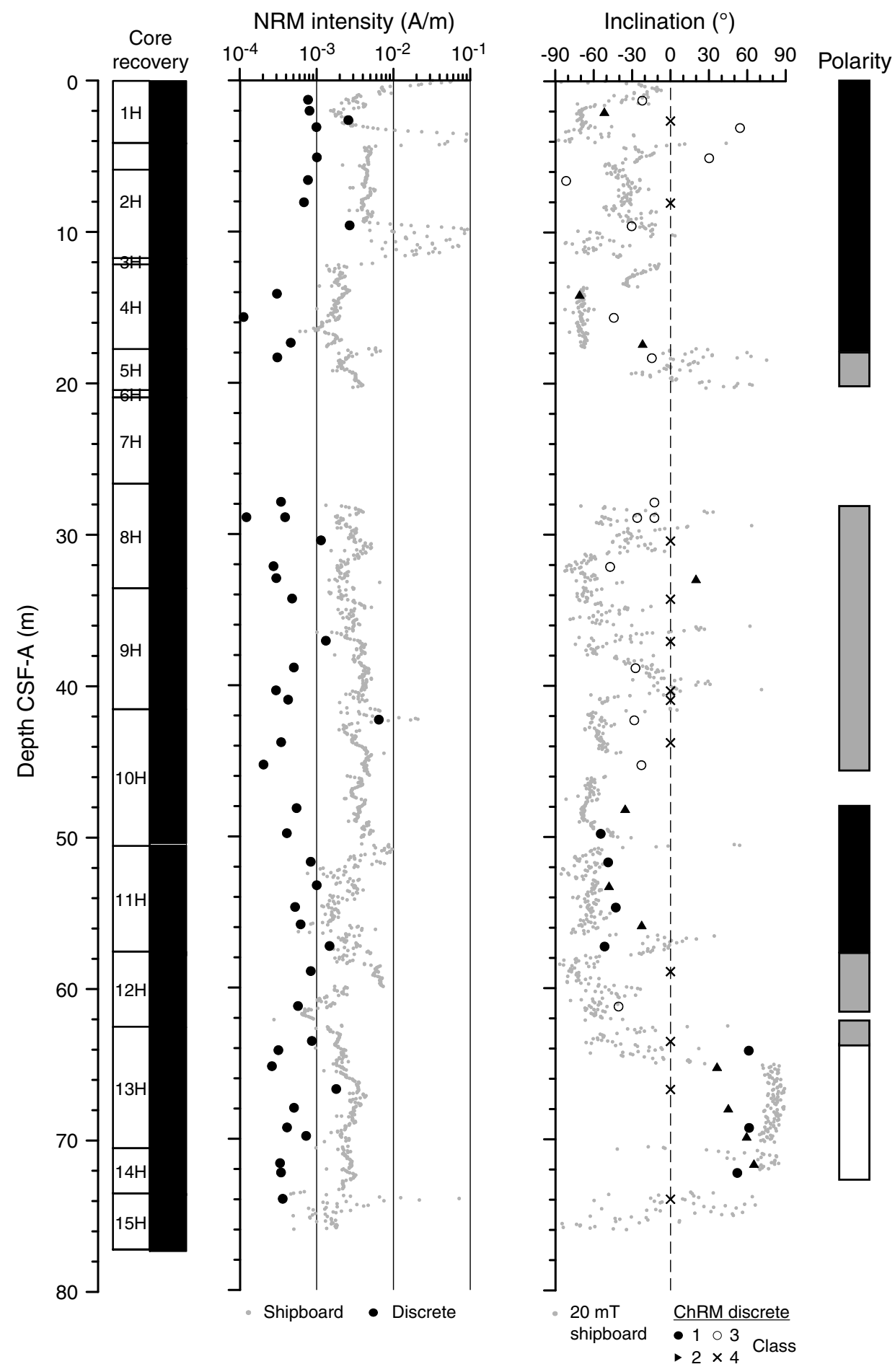


Figure F12. Natural remanenent magnetization (NRM) intensity and characteristic remanent magnetization (ChRM) inclination for discrete samples from 60 to $300 \mathrm{~m}$ CSF-A in Hole U1354C. Shipboard section-half NRM intensities and ChRM inclinations after $20 \mathrm{mT}$ AF demagnetization are also plotted. The quality of the demagnetization data on the discrete samples has been ranked in four different classes (Class $1=$ higher quality, Class $4=$ no interpretation possible; see text for explanation). Polarity indicates the inferred ChRM polarity based only on Class 1 and 2 samples (normal polarity for negative inclination and reverse polarity for positive inclination).

Hole U1354C

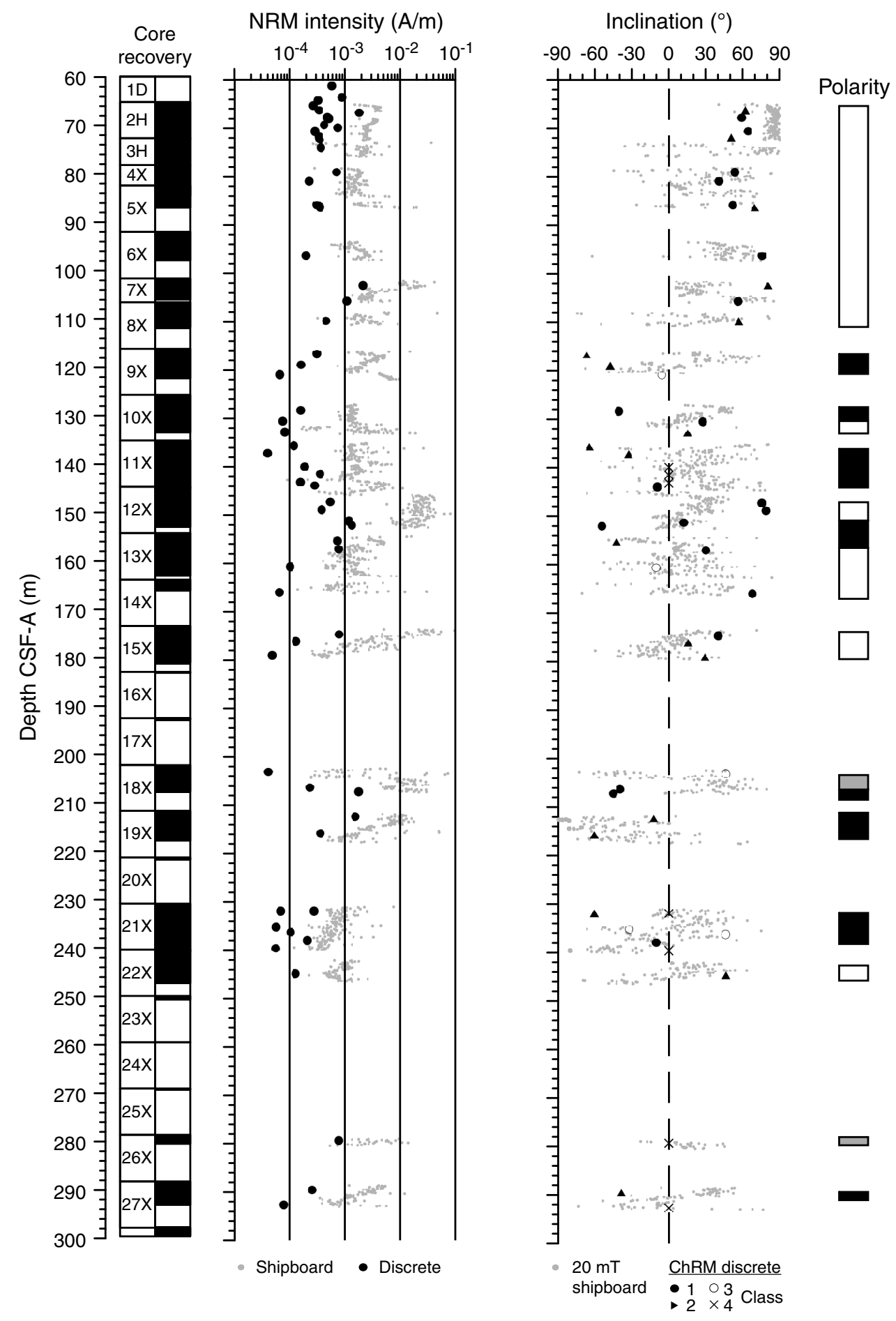


Table T1. Paleomagnetic demagnetization data of discrete samples, Hole U1351B. (Continued on next page.)

\begin{tabular}{|c|c|c|c|c|c|c|c|c|}
\hline $\begin{array}{l}\text { Core, section, } \\
\text { interval }(\mathrm{cm})\end{array}$ & $\begin{array}{c}\text { Depth } \\
\text { CSF-A (m) }\end{array}$ & Lab & Treatment & $\begin{array}{c}\text { NRM } \\
\text { intensity } \\
(\mathrm{A} / \mathrm{m})\end{array}$ & $\begin{array}{c}\text { ChRM } \\
\text { inclination } \\
\left({ }^{\circ}\right)\end{array}$ & $\begin{array}{c}\text { MAD } \\
\left({ }^{\circ}\right)\end{array}$ & Steps & Class \\
\hline \multicolumn{9}{|l|}{ 317-U1351B- } \\
\hline 1H-1, 89-91 & 0.91 & $\mathrm{RM}$ & $\mathrm{AF}$ & $1.03 \mathrm{E}-03$ & & & & 4 \\
\hline $1 \mathrm{H}-2,89-91$ & 2.41 & RM & $\mathrm{AF}$ & $7.32 \mathrm{E}-04$ & & & & 4 \\
\hline $1 \mathrm{H}-3,51-53$ & 3.53 & $\mathrm{RM}$ & $\mathrm{AF}$ & $1.39 \mathrm{E}-04$ & -61.33 & 12.6 & 30 -or & 3 \\
\hline $1 \mathrm{H}-4,53-55$ & 5.05 & RM & $\mathrm{AF}$ & $2.16 \mathrm{E}-04$ & -57.33 & 11.7 & 21 -or & 2 \\
\hline $1 \mathrm{H}-5,48-50$ & 6.50 & $\mathrm{RM}$ & $\mathrm{AF}$ & $1.29 \mathrm{E}-04$ & -67.90 & 17.5 & 20 -or & 3 \\
\hline $2 \mathrm{H}-1,91-93$ & 8.73 & $\mathrm{RM}$ & $\mathrm{AF}$ & $4.96 \mathrm{E}-04$ & -57.31 & 8.8 & 25 -or & 1 \\
\hline $2 \mathrm{H}-2,103-105$ & 10.35 & $\mathrm{RM}$ & $\mathrm{AF}$ & $6.56 \mathrm{E}-04$ & -44.84 & 6.0 & 25 -or & 1 \\
\hline $2 \mathrm{H}-3,76-78$ & 11.58 & $\mathrm{RM}$ & $\mathrm{AF}$ & $3.21 \mathrm{E}-04$ & -20.34 & 21.3 & 40 -or & 3 \\
\hline $2 \mathrm{H}-4,116-118$ & 13.48 & $\mathrm{RM}$ & $\mathrm{AF}$ & $6.33 \mathrm{E}-04$ & -47.79 & 19.5 & 25 -or & 2 \\
\hline $2 \mathrm{H}-6,92-94$ & 16.24 & $\mathrm{RM}$ & $\mathrm{AF}$ & $7.00 \mathrm{E}-04$ & 35.00 & 6.3 & 30-or & 2 \\
\hline $3 \mathrm{H}-1,47-49$ & 17.19 & $\mathrm{RM}$ & $\mathrm{AF}$ & $8.21 \mathrm{E}-05$ & -42.12 & 18.7 & $21-35$ & 2 \\
\hline $3 \mathrm{H}-2,49-51$ & 18.71 & $\mathrm{RM}$ & $\mathrm{AF}$ & $2.15 \mathrm{E}-04$ & -22.88 & 22.7 & $17-30$ & 3 \\
\hline $3 \mathrm{H}-3,53-55$ & 20.25 & $\mathrm{RM}$ & $\mathrm{AF}$ & $4.53 \mathrm{E}-04$ & -53.06 & 7.6 & 30-or & 1 \\
\hline $3 \mathrm{H}-4,4-6$ & 21.26 & $\mathrm{RM}$ & $\mathrm{AF}$ & $3.33 \mathrm{E}-04$ & -45.12 & 30.9 & 25 -or & 3 \\
\hline $4 \mathrm{H}-1,47-49$ & 22.59 & $\mathrm{RM}$ & $\mathrm{AF}$ & $1.20 \mathrm{E}-04$ & -44.71 & 13.5 & 17-or & 3 \\
\hline $4 \mathrm{H}-2,118-120$ & 24.80 & $\mathrm{RM}$ & $\mathrm{AF}$ & $1.97 \mathrm{E}-04$ & -42.02 & 14.0 & 21 -or & 3 \\
\hline $4 \mathrm{H}-3,119-121$ & 26.31 & $\mathrm{RM}$ & $\mathrm{AF}$ & $1.45 \mathrm{E}-04$ & -29.22 & 16.0 & 17-or & 3 \\
\hline $4 \mathrm{H}-4,107-109$ & 27.69 & $\mathrm{RM}$ & $\mathrm{AF}$ & $5.53 \mathrm{E}-04$ & & & & 4 \\
\hline $5 \mathrm{H}-2,41-43$ & 29.96 & $\mathrm{RM}$ & $\mathrm{AF}$ & $3.81 \mathrm{E}-04$ & 35.21 & 13.2 & 35 -or & 2 \\
\hline $6 \mathrm{H}-2,8-10$ & 33.80 & $\mathrm{RM}$ & $\mathrm{AF}$ & $4.68 \mathrm{E}-04$ & 8.03 & 6.2 & 35 -or & 3 \\
\hline $6 \mathrm{H}-3,68-70$ & 35.90 & $\mathrm{RM}$ & $\mathrm{AF}$ & $4.04 \mathrm{E}-04$ & 25.88 & 17.4 & 30 -or & 3 \\
\hline $6 \mathrm{H}-4,73-75$ & 37.45 & $\mathrm{RM}$ & $\mathrm{AF}$ & $1.22 \mathrm{E}-03$ & 30.18 & 12.4 & 21-or & 2 \\
\hline $6 \mathrm{H}-5,38-40$ & 38.60 & $\mathrm{RM}$ & $\mathrm{AF}$ & $3.58 \mathrm{E}-04$ & -48.81 & 19.0 & 17-or & 3 \\
\hline $7 \mathrm{H}-3,54-56$ & 42.76 & $\mathrm{RM}$ & $\mathrm{AF}$ & $4.57 \mathrm{E}-04$ & -14.53 & 17.5 & 30 -or & 2 \\
\hline $7 \mathrm{H}-4,38-40$ & 44.10 & $\mathrm{RM}$ & $\mathrm{AF}$ & $3.49 \mathrm{E}-04$ & -44.31 & 31.1 & 30 -or & 3 \\
\hline $7 \mathrm{H}-5,45-47$ & 45.67 & $\mathrm{RM}$ & $\mathrm{AF}$ & $4.75 \mathrm{E}-04$ & -23.26 & 26.1 & 21-or & 3 \\
\hline $7 \mathrm{H}-6,36-38$ & 47.08 & $\mathrm{RM}$ & $\mathrm{AF}$ & $5.34 \mathrm{E}-04$ & -15.56 & 25.3 & $25-35$ & 3 \\
\hline $8 \mathrm{H}-1,73-75$ & 48.45 & RM & $\mathrm{AF}$ & $5.47 \mathrm{E}-04$ & -22.36 & 21.2 & $13-30$ & 3 \\
\hline $8 \mathrm{H}-2,72-74$ & 49.94 & $\mathrm{RM}$ & $\mathrm{AF}$ & $5.30 \mathrm{E}-04$ & & & & 4 \\
\hline $8 \mathrm{H}-3,72-74$ & 51.44 & $\mathrm{RM}$ & $\mathrm{AF}$ & $3.95 \mathrm{E}-04$ & -9.32 & 20.7 & 25 -or & 3 \\
\hline $8 \mathrm{H}-4,70-72$ & 52.92 & $\mathrm{RM}$ & $\mathrm{AF}$ & $3.25 \mathrm{E}-04$ & -21.09 & 19.5 & $13-60$ & 3 \\
\hline $8 \mathrm{H}-5,74-76$ & 54.46 & OT & $\mathrm{AF}$ & $8.85 \mathrm{E}-01$ & & & & 4 \\
\hline $8 \mathrm{H}-6,74-76$ & 55.96 & $\mathrm{RM}$ & $\mathrm{AF}$ & $4.38 \mathrm{E}-04$ & -52.34 & 14.3 & 35 -or & 2 \\
\hline $9 \mathrm{H}-1,102-104$ & 58.24 & $\mathrm{RM}$ & $\mathrm{AF}$ & $5.02 \mathrm{E}-04$ & -62.48 & 6.1 & 17-or & 1 \\
\hline $9 \mathrm{H}-2,102-104$ & 59.74 & $\mathrm{RM}$ & $\mathrm{AF}$ & $7.00 \mathrm{E}-04$ & -23.15 & 10.4 & 17-or & 2 \\
\hline $9 \mathrm{H}-3,99-101$ & 61.21 & RM & $\mathrm{AF}$ & $2.69 \mathrm{E}-03$ & -26.52 & 2.6 & 25 -or & 1 \\
\hline $9 \mathrm{H}-5,89-91$ & 64.11 & $\mathrm{RM}$ & $\mathrm{AF}$ & $1.67 \mathrm{E}-04$ & -71.33 & 14.5 & 21-or & 1 \\
\hline $9 \mathrm{H}-6,50-52$ & 65.22 & $\mathrm{RM}$ & $\mathrm{AF}$ & $1.42 \mathrm{E}-04$ & -59.60 & 33.6 & 17-or & 3 \\
\hline $11 \mathrm{H}-1,116-118$ & 70.88 & $\mathrm{RM}$ & $\mathrm{AF}$ & $2.23 \mathrm{E}-04$ & 73.40 & 7.9 & 21-or & 2 \\
\hline $11 \mathrm{H}-3,48-50$ & 73.20 & $\mathrm{RM}$ & $\mathrm{AF}$ & $1.00 \mathrm{E}-04$ & 44.78 & 18.7 & 21 -or & 2 \\
\hline $11 \mathrm{H}-4,26-28$ & 74.48 & $\mathrm{RM}$ & $\mathrm{AF}$ & $1.94 \mathrm{E}-04$ & & & & 4 \\
\hline $13 \mathrm{H}-1,75-77$ & 85.97 & $\mathrm{RM}$ & $\mathrm{AF}$ & $1.82 \mathrm{E}-04$ & 48.57 & 14.9 & 45 -or & 3 \\
\hline $13 \mathrm{H}-2,17-19$ & 86.37 & $\mathrm{RM}$ & $\mathrm{AF}$ & $1.25 \mathrm{E}-04$ & 50.28 & 9.9 & 30-or & 1 \\
\hline $13 \mathrm{H}-3,122-124$ & 88.92 & OT & $\mathrm{AF}$ & $3.38 \mathrm{E}-04$ & 51.8 & 6.6 & 400 -or & 1 \\
\hline $14 X-2,24-26$ & 96.46 & $\mathrm{RM}$ & $\mathrm{AF}$ & $7.63 \mathrm{E}-04$ & 69.39 & 2.0 & 25-or & 1 \\
\hline $16 X-1,127-129$ & 114.19 & $\mathrm{RM}$ & $\mathrm{AF}$ & $1.92 \mathrm{E}-04$ & -22.21 & 23.9 & 40-or & 3 \\
\hline $16 X-2,109-111$ & 115.51 & OT & $\mathrm{AF}$ & $4.83 \mathrm{E}-04$ & 44.1 & 8.5 & 30-or & 2 \\
\hline $16 X-3,26-28$ & 116.18 & $\mathrm{RM}$ & $\mathrm{AF}$ & $2.22 \mathrm{E}-04$ & 32.84 & 19.6 & 25 -or & 2 \\
\hline $16 X-4,69-70$ & 118.10 & $\mathrm{RM}$ & $\mathrm{AF}$ & $2.38 \mathrm{E}-04$ & 27.50 & 20.5 & 35-or & 2 \\
\hline $16 X-5,72-74$ & 119.64 & $\mathrm{RM}$ & $\mathrm{AF}$ & $3.95 \mathrm{E}-04$ & 51.70 & 15.7 & 25 -or & 3 \\
\hline $18 X-1,91-92$ & 132.92 & От & $\mathrm{AF}$ & $2.96 \mathrm{E}-04$ & & & & 4 \\
\hline $18 \times-2,17-19$ & 133.69 & $\mathrm{RM}$ & $\mathrm{AF}$ & $2.93 \mathrm{E}-04$ & 22.47 & 7.5 & 35-or & 1 \\
\hline $19 X-1,102-104$ & 142.64 & $\mathrm{RM}$ & $\mathrm{AF}$ & $1.16 \mathrm{E}-04$ & & & & 4 \\
\hline $19 X-3,117-119$ & 145.79 & $\mathrm{RM}$ & $\mathrm{AF}$ & $2.29 \mathrm{E}-04$ & -77.24 & 3.0 & 40-or & 1 \\
\hline $19 X-4,69-71$ & 146.81 & $\mathrm{RM}$ & $\mathrm{AF}$ & $1.36 \mathrm{E}-04$ & -56.97 & 2.2 & 40-or & 1 \\
\hline $20 x-1,74-76$ & 151.96 & $\mathrm{RM}$ & $\mathrm{AF}$ & $6.55 \mathrm{E}-04$ & 60.21 & 5.0 & 35-or & 1 \\
\hline $20 \times-2,96-98$ & 153.68 & $\mathrm{RM}$ & $\mathrm{AF}$ & $7.34 \mathrm{E}-05$ & 28.16 & 8.7 & 21-or & 1 \\
\hline $20 \times-4,96-98$ & 156.68 & $\mathrm{RM}$ & $\mathrm{AF}$ & $1.61 \mathrm{E}-03$ & 74.23 & 10.5 & 25-or & 1 \\
\hline $20 X-5,70-72$ & 157.92 & $\mathrm{RM}$ & $\mathrm{AF}$ & $2.40 \mathrm{E}-03$ & 49.51 & 5.5 & 40-or & 1 \\
\hline $20 X-6,49-51$ & 159.21 & $\mathrm{RM}$ & $\mathrm{AF}$ & $2.55 \mathrm{E}-03$ & 85.71 & 4.6 & 21-or & 1 \\
\hline $21 X-1,71-73$ & 161.53 & $\mathrm{RM}$ & $\mathrm{AF}$ & $4.13 \mathrm{E}-03$ & 53.36 & 7.4 & 30-or & 1 \\
\hline $21 X-2,88-90$ & 163.20 & $\mathrm{RM}$ & $\mathrm{AF}$ & $6.49 \mathrm{E}-03$ & 49.42 & 5.1 & 21-or & 1 \\
\hline $21 X-3,88-90$ & 164.70 & От & $\mathrm{AF}$ & $6.48 \mathrm{E}-02$ & 52.9 & 6.4 & 20-or & 1 \\
\hline $21 X-4,38-40$ & 165.70 & $\mathrm{RM}$ & $\mathrm{AF}$ & $6.51 \mathrm{E}-03$ & 59.41 & 8.2 & 17-or & 1 \\
\hline $21 X-5,72-74$ & 167.54 & $\mathrm{RM}$ & $\mathrm{AF}$ & $9.58 \mathrm{E}-04$ & 52.51 & 12.8 & 30-or & 1 \\
\hline $22 X-2,57-59$ & 172.49 & $\mathrm{RM}$ & $\mathrm{AF}$ & $7.09 \mathrm{E}-05$ & -66.68 & 16.0 & 45-or & 3 \\
\hline $25 X-1,48-50$ & 199.70 & OT & $\mathrm{AF}$ & $2.28 \mathrm{E}-04$ & 1.5 & 3.0 & 25 -or & 2 \\
\hline
\end{tabular}


Table T1 (continued).

\begin{tabular}{|c|c|c|c|c|c|c|c|c|}
\hline $\begin{array}{l}\text { Core, section, } \\
\text { interval }(\mathrm{cm})\end{array}$ & $\begin{array}{l}\text { Depth } \\
\text { CSF-A (m) }\end{array}$ & Lab & Treatment & $\begin{array}{c}\text { NRM } \\
\text { intensity } \\
(A / m)\end{array}$ & $\begin{array}{c}\text { ChRM } \\
\text { inclination } \\
\left(^{\circ}\right)\end{array}$ & $\begin{array}{l}\text { MAD } \\
\left({ }^{\circ}\right)\end{array}$ & Steps & Class \\
\hline $27 X-1,87-89$ & 219.29 & RM & $\mathrm{AF}$ & 4.47E-05 & 38.33 & 9.3 & 21 -or & 1 \\
\hline $27 X-2,91-94$ & 220.84 & OT & $\mathrm{AF}$ & $1.46 \mathrm{E}-03$ & -47.2 & 3.4 & 45 -or & 1 \\
\hline $27 X-3,87-89$ & 222.29 & RM & $\mathrm{AF}$ & $3.38 \mathrm{E}-04$ & 65.46 & 12.7 & 40 -or & 1 \\
\hline $27 X-4,40-42$ & 223.32 & RM & $\mathrm{AF}$ & $4.81 \mathrm{E}-04$ & 64.05 & 8.1 & 35 -or & 1 \\
\hline $27 X-6,32-34$ & 225.84 & RM & $\mathrm{AF}$ & $4.70 \mathrm{E}-04$ & 5.63 & 10.4 & 21 -or & 2 \\
\hline $28 \mathrm{X}-1,123-125$ & 229.25 & RM & $\mathrm{AF}$ & $4.44 \mathrm{E}-04$ & -14.64 & 10.6 & 21 -or & 2 \\
\hline $28 X-2,68-70$ & 230.20 & RM & $\mathrm{AF}$ & $1.64 \mathrm{E}-04$ & -65.36 & 10.1 & 45 -or & 2 \\
\hline $28 \mathrm{X}-4,120-122$ & 233.72 & RM & $\mathrm{AF}$ & $6.10 \mathrm{E}-04$ & -39.18 & 12.9 & 30 -or & 2 \\
\hline $28 X-5,84-86$ & 234.86 & RM & AF & $6.23 \mathrm{E}-04$ & & & & 4 \\
\hline $28 X-6,40-42$ & 235.62 & RM & $\mathrm{AF}$ & $3.31 \mathrm{E}-04$ & -25.82 & 27.7 & 25 -or & 2 \\
\hline $29 X-1,85-87$ & 238.37 & RM & $\mathrm{AF}$ & 4.67E-04 & -71.38 & 6.9 & 30 -or & 1 \\
\hline $29 X-2,81-83$ & 239.83 & RM & $\mathrm{AF}$ & $4.20 \mathrm{E}-04$ & 15.89 & 20.5 & 35 -or & 3 \\
\hline $29 X-3,35-37$ & 240.87 & RM & $\mathrm{AF}$ & $4.53 \mathrm{E}-04$ & -50.93 & 9.4 & 21 -or & 1 \\
\hline $29 X-4,35-37$ & 242.37 & RM & $\mathrm{AF}$ & $4.64 \mathrm{E}-04$ & -35.60 & 20.9 & 13 -or & 3 \\
\hline $28 X-5,38-40$ & 243.90 & OT & $\mathrm{AF}$ & $1.08 \mathrm{E}-03$ & -41.4 & 18.6 & 20 -or & 3 \\
\hline $29 X-6,39-41$ & 245.41 & RM & $\mathrm{AF}$ & $9.97 \mathrm{E}-04$ & 30.12 & 22.9 & 17 -or & 3 \\
\hline $29 X-7,14-16$ & 246.66 & RM & $\mathrm{AF}$ & $2.96 \mathrm{E}-04$ & -33.47 & 18.1 & 35 -or & 2 \\
\hline $30 X-6,30-32$ & 254.52 & RM & $\mathrm{AF}$ & $5.08 \mathrm{E}-04$ & 45.61 & 5.6 & 35 -or & 1 \\
\hline $30 X-6,40-42$ & 254.62 & OT & $\mathrm{AF}$ & $5.38 \mathrm{E}-04$ & 50.3 & 12.9 & 25 -or & 3 \\
\hline $31 X-3,103-105$ & 260.75 & RM & $\mathrm{AF}$ & $1.95 \mathrm{E}-04$ & 35.61 & 3.8 & 45 -or & 1 \\
\hline $31 X-4,51-53$ & 261.73 & RM & $\mathrm{AF}$ & $1.02 \mathrm{E}-04$ & 39.01 & 5.4 & 35 -or & 1 \\
\hline $31 X-5,31-33$ & 263.03 & OT & $\mathrm{AF}$ & $2.20 \mathrm{E}-04$ & & & & 4 \\
\hline $32 X-1,106-108$ & 267.38 & RM & $\mathrm{AF}$ & $1.01 \mathrm{E}-04$ & -13.21 & 16.1 & 30 -or & 3 \\
\hline $32 X-2,87-89$ & 268.69 & OT & $\mathrm{AF}$ & $2.64 \mathrm{E}-04$ & 67.8 & 8.6 & 25 -or & 2 \\
\hline $32 X-3,108-110$ & 270.40 & RM & $\mathrm{AF}$ & $3.38 \mathrm{E}-05$ & -35.34 & 23.1 & 21 -or & 3 \\
\hline $36 X-2,46-48$ & 306.68 & RM & $\mathrm{AF}$ & $6.51 \mathrm{E}-03$ & -23.67 & 21.6 & 17 -or & 3 \\
\hline $36 \mathrm{X}-3,84-86$ & 308.56 & RM & $\mathrm{AF}$ & $8.26 \mathrm{E}-05$ & & & & 4 \\
\hline $37 X-1,115-117$ & 315.07 & RM & $\mathrm{AF}$ & $4.45 \mathrm{E}-05$ & & & & 4 \\
\hline $37 X-2,98-100$ & 316.40 & OT & $\mathrm{AF}$ & $2.04 \mathrm{E}-04$ & & & & 4 \\
\hline $39 X-3,40-42$ & 336.52 & RM & AF & $1.98 \mathrm{E}-04$ & 39.25 & 8.9 & 17 -or & 1 \\
\hline $40 \times-2,49-51$ & 344.71 & OT & $\mathrm{AF}$ & $2.59 \mathrm{E}-04$ & & & & 4 \\
\hline $41 X-1,89-91$ & 353.21 & RM & $\mathrm{AF}$ & $8.58 \mathrm{E}-05$ & & & & 4 \\
\hline $45 X-2,29-31$ & 392.51 & RM & $\mathrm{AF}$ & $6.71 \mathrm{E}-04$ & & & & 4 \\
\hline $45 X-2,33-35$ & 392.55 & OT & $\mathrm{AF}$ & $2.33 \mathrm{E}-04$ & 16.1 & 5.7 & 20 -or & 3 \\
\hline $47 X-1,49-51$ & 410.31 & RM & $\mathrm{AF}$ & $5.37 \mathrm{E}-05$ & -14.52 & 20.9 & 30 -or & 3 \\
\hline $64 \mathrm{X}-1,118-120$ & 554.60 & RM & $\mathrm{AF}$ & $6.48 \mathrm{E}-04$ & 40.04 & 14.1 & 17-or & 3 \\
\hline $64 X-2,84-86$ & 555.76 & OT & $\mathrm{AF}$ & $1.34 \mathrm{E}-03$ & & & & 4 \\
\hline $70 X-1,113-115$ & 602.65 & OT & $\mathrm{AF}$ & $2.32 \mathrm{E}-04$ & 53.4 & 5.4 & 20 -or & 3 \\
\hline $70 X-2,17-19$ & 602.99 & RM & $\mathrm{AF}$ & $1.31 \mathrm{E}-04$ & 24.09 & 10.6 & 17 -or & 1 \\
\hline $72 X-1,91-93$ & 621.53 & OT & $\mathrm{AF}$ & $9.10 \mathrm{E}-05$ & -19.4 & 12.7 & 30 -or & 3 \\
\hline $72 X-2,76-78$ & 622.88 & RM & $\mathrm{AF}$ & $2.61 \mathrm{E}-04$ & 23.42 & 12.4 & 30 -or & 1 \\
\hline $73 X-1,73-75$ & 630.95 & $\mathrm{RM}$ & $\mathrm{AF}$ & $5.88 \mathrm{E}-05$ & -30.76 & 10.7 & 30 -or & 3 \\
\hline $88 X-1,86-88$ & 755.78 & OT & $\mathrm{AF}$ & $3.47 \mathrm{E}-04$ & 14.4 & 5.4 & 25 -or & 3 \\
\hline 89X-1, 89-91 & 765.41 & OT & $\mathrm{AF}$ & $1.26 \mathrm{E}-04$ & & & & 4 \\
\hline $90 \mathrm{X}-2,136-138$ & 776.98 & RM & AF & $1.99 \mathrm{E}-04$ & -25.79 & 14.6 & 50 -or & 3 \\
\hline $90 X-3,84-86$ & 777.96 & OT & $\mathrm{AF}$ & $1.78 \mathrm{E}-04$ & & & & 4 \\
\hline 92X-1, 91-93 & 794.23 & RM & $\mathrm{AF}$ & $2.58 \mathrm{E}-05$ & -61.39 & 14.9 & 35 -or & 2 \\
\hline $92 X-2,74-76$ & 795.56 & OT & $\mathrm{AF}$ & $1.33 \mathrm{E}-04$ & & & & 4 \\
\hline $93 X-2,46-48$ & 804.98 & RM & $\mathrm{AF}$ & 1.37E-04 & 49.66 & 9.0 & 21 -or & 1 \\
\hline $94 X-1,50-52$ & 813.12 & RM & $\mathrm{AF}$ & $1.04 \mathrm{E}-04$ & 19.85 & 11.7 & 35 -or & 3 \\
\hline $94 X-3,50-52$ & 816.12 & OT & AF & $2.45 \mathrm{E}-04$ & & & & 4 \\
\hline $97 X-2,85-87$ & 843.77 & OT & $\mathrm{AF}$ & $2.03 \mathrm{E}-04$ & & & & 4 \\
\hline $97 X-3,88-90$ & 845.30 & RM & $\mathrm{AF}$ & $7.10 \mathrm{E}-05$ & & & & 4 \\
\hline $100 \mathrm{X}-2,100-102$ & 872.82 & RM & AF & $2.70 \mathrm{E}-05$ & & & & 4 \\
\hline $100 \times-3,104-106$ & 874.36 & OT & $\mathrm{AF}$ & $2.82 \mathrm{E}-04$ & & & & 4 \\
\hline $100 \times-4,105-107$ & 875.87 & RM & $\mathrm{AF}$ & $9.44 \mathrm{E}-05$ & -59.68 & 30.7 & 35 -or & 3 \\
\hline $101 X-1,85-87$ & 880.77 & RM & $\mathrm{AF}$ & $2.01 \mathrm{E}-04$ & & & & 4 \\
\hline $101 X-2,51-53$ & 881.93 & OT & $\mathrm{AF}$ & $2.18 \mathrm{E}-04$ & & & & 4 \\
\hline $104 \mathrm{X}-1,123-125$ & 910.05 & RM & $\mathrm{AF}$ & $1.70 \mathrm{E}-04$ & & & & 4 \\
\hline $104 X-2,106-108$ & 911.38 & OT & $\mathrm{AF}$ & $4.15 \mathrm{E}-04$ & & & & 4 \\
\hline $106 \times-2,33-35$ & 929.85 & OT & $\mathrm{AF}$ & $1.18 \mathrm{E}-03$ & & & & 4 \\
\hline $109 \times-2,52-54$ & 958.84 & OT & $\mathrm{AF}$ & $6.68 \mathrm{E}-04$ & & & & 4 \\
\hline $109 \times-3,73-75$ & 960.55 & $\mathrm{RM}$ & $\mathrm{AF}$ & $2.00 \mathrm{E}-04$ & & & & 4 \\
\hline $112 X-3,61-63$ & 989.13 & OT & $\mathrm{AF}$ & $1.87 \mathrm{E}-04$ & & & & 4 \\
\hline
\end{tabular}

$\mathrm{RM}=$ Rome laboratory, $\mathrm{OT}=$ Otago laboratory. $\mathrm{AF}=$ alternating field demagnetization. $\mathrm{NRM}=$ natural remanent magnetization, $\mathrm{ChRM}=\mathrm{charac}-$ teristic remanent magnetization. MAD = maximum angular deviation. Steps: demagnetization steps included for ChRM calculation in $\mathrm{mT}$ for $\mathrm{AF}$, or $=$ anchored to the origin. Class $=$ quality ranking for demagnetization data: $1=$ high, $2=$ intermediate quality, $3=$ unreliable, $4=$ no direction calculated. 
Table T2. Paleomagnetic demagnetization data of discrete samples, Holes U1352B and U1352C. (Continued on next seven pages.)

\begin{tabular}{|c|c|c|c|c|c|c|c|c|}
\hline $\begin{array}{l}\text { Core, section, } \\
\text { interval }(\mathrm{cm})\end{array}$ & $\begin{array}{c}\text { Depth } \\
\text { CSF-A (m) }\end{array}$ & Lab & Treatment & $\begin{array}{c}\text { NRM } \\
\text { intensity } \\
(\mathrm{A} / \mathrm{m})\end{array}$ & $\begin{array}{c}\text { ChRM } \\
\text { inclination } \\
\left({ }^{\circ}\right)\end{array}$ & $\begin{array}{c}\text { MAD } \\
\left({ }^{\circ}\right)\end{array}$ & Steps & Class \\
\hline \multicolumn{9}{|l|}{ 317-U1352B- } \\
\hline $1 \mathrm{H}-1,82-84$ & 0.84 & $\mathrm{RM}$ & $\mathrm{AF}$ & $5.15 \mathrm{E}-04$ & -31.99 & 15.2 & 13-or & 2 \\
\hline $1 \mathrm{H}-3,108-110$ & 4.08 & OT & $\mathrm{AF}$ & $3.67 \mathrm{E}-04$ & & & & 4 \\
\hline $1 \mathrm{H}-4,134-136$ & 5.86 & $\mathrm{RM}$ & $\mathrm{AF}$ & $1.07 \mathrm{E}-03$ & & & & 4 \\
\hline $2 \mathrm{H}-2,104-106$ & 10.76 & $\mathrm{RM}$ & $\mathrm{AF}$ & $5.83 \mathrm{E}-04$ & -23.22 & 8.3 & 17-or & 2 \\
\hline $2 \mathrm{H}-4,124-126$ & 13.96 & $\mathrm{RM}$ & $\mathrm{AF}$ & $3.12 \mathrm{E}-04$ & -81.02 & 16.9 & 21 -or & 3 \\
\hline $3 \mathrm{H}-1,19-21$ & 19.06 & $\mathrm{RM}$ & $\mathrm{AF}$ & $1.18 \mathrm{E}-03$ & -60.94 & 17.2 & 17-or & 2 \\
\hline $3 \mathrm{H}-2,124-126$ & 20.46 & OT & $\mathrm{AF}$ & $1.08 \mathrm{E}-03$ & -44.50 & 14.0 & 25 -or & 2 \\
\hline $3 \mathrm{H}-4,127-129$ & 23.49 & $\mathrm{RM}$ & $\mathrm{AF}$ & $1.25 \mathrm{E}-03$ & -29.29 & 10.3 & 30 -or & 2 \\
\hline $3 \mathrm{H}-5,90-92$ & 24.62 & $\mathrm{RM}$ & $\mathrm{AF}$ & $1.72 \mathrm{E}-02$ & -39.17 & 35.5 & $17-60$ & 2 \\
\hline $3 \mathrm{H}-6,40-42$ & 25.62 & $\mathrm{RM}$ & $\mathrm{AF}$ & $4.06 \mathrm{E}-04$ & -33.95 & 23.6 & 13-or & 3 \\
\hline $5 \mathrm{H}-2,14-16$ & 37.08 & $\mathrm{RM}$ & $\mathrm{AF}$ & $3.47 \mathrm{E}-03$ & -64.99 & 10.8 & 17-or & 1 \\
\hline $5 \mathrm{H}-6,26-28$ & 43.20 & OT & $\mathrm{AF}$ & $4.21 \mathrm{E}-03$ & -15.40 & 7.1 & 35 -or & 2 \\
\hline $6 \mathrm{H}-3,41-43$ & 49.63 & $\mathrm{RM}$ & $\mathrm{AF}$ & $1.14 \mathrm{E}-03$ & -55.89 & 15.2 & 17-or & 1 \\
\hline $6 \mathrm{H}-4,22-24$ & 50.94 & OT & $\mathrm{AF}$ & $2.18 \mathrm{E}-03$ & -47.90 & 5.2 & 30 -or & 1 \\
\hline $6 \mathrm{H}-5,23-25$ & 52.45 & RM & $\mathrm{AF}$ & $2.63 \mathrm{E}-03$ & -72.53 & 9.8 & 17-or & 2 \\
\hline $6 \mathrm{H}-6,21-24$ & 53.94 & RM & $\mathrm{AF}$ & $1.66 \mathrm{E}-02$ & -45.59 & 6.9 & $21-50$ & 1 \\
\hline $7 \mathrm{H}-1,49-51$ & 56.21 & $\mathrm{RM}$ & $\mathrm{AF}$ & $1.53 \mathrm{E}-03$ & -64.23 & 10.3 & 40 -or & 2 \\
\hline $7 \mathrm{H}-2,62-64$ & 57.84 & $\mathrm{RM}$ & $\mathrm{AF}$ & $3.48 \mathrm{E}-03$ & -58.77 & 12.0 & 25 -or & 2 \\
\hline $7 \mathrm{H}-4,52-54$ & 60.74 & OT & $\mathrm{AF}$ & $1.40 \mathrm{E}-02$ & & & & 4 \\
\hline 7H-5, 100-102 & 62.72 & RM & $\mathrm{AF}$ & $2.10 \mathrm{E}-03$ & -54.01 & 9.8 & 21 -or & 2 \\
\hline $8 \mathrm{H}-1,98-100$ & 66.20 & $\mathrm{RM}$ & $\mathrm{AF}$ & $8.35 \mathrm{E}-04$ & -50.84 & 12.2 & 25 -or & 1 \\
\hline $8 \mathrm{H}-2,118-120$ & 67.90 & $\mathrm{RM}$ & $\mathrm{AF}$ & $3.00 \mathrm{E}-04$ & -54.53 & 5.1 & 21 -or & 2 \\
\hline $8 \mathrm{H}-3,91-93$ & 69.13 & $\mathrm{RM}$ & $\mathrm{AF}$ & $5.85 \mathrm{E}-04$ & -67.62 & 8.0 & 17-or & 2 \\
\hline $8 \mathrm{H}-5,69-71$ & 71.91 & $\mathrm{RM}$ & $\mathrm{AF}$ & $1.53 \mathrm{E}-03$ & -73.41 & 2.7 & 21 -or & 2 \\
\hline $8 \mathrm{H}-6,31-33$ & 73.03 & $\mathrm{RM}$ & $\mathrm{AF}$ & $5.77 \mathrm{E}-04$ & -73.17 & 7.4 & 21 -or & 2 \\
\hline $9 \mathrm{H}-1,113-115$ & 75.85 & $\mathrm{RM}$ & $\mathrm{AF}$ & $6.10 \mathrm{E}-04$ & -63.22 & 7.9 & 13-or & 2 \\
\hline $9 \mathrm{H}-3,54-56$ & 78.24 & От & $\mathrm{AF}$ & $1.81 \mathrm{E}-03$ & -71.20 & 5.2 & 20 -or & 1 \\
\hline $9 \mathrm{H}-3,54-56$ & 78.26 & OT & $\mathrm{AF}$ & $1.64 \mathrm{E}-03$ & -50.70 & 5.6 & 25 -or & 1 \\
\hline $9 \mathrm{H}-5,60-62$ & 81.32 & RM & $\mathrm{AF}$ & $8.65 \mathrm{E}-04$ & -20.68 & 16.6 & 17-or & 3 \\
\hline $9 \mathrm{H}-7,29-31$ & 83.51 & $\mathrm{RM}$ & $\mathrm{AF}$ & $5.99 \mathrm{E}-04$ & -84.91 & 0.8 & 45 -or & 1 \\
\hline $10 \mathrm{H}-3,41-43$ & 86.39 & $\mathrm{RM}$ & $\mathrm{AF}$ & 4.77E-04 & -31.08 & 6.3 & 17-or & 2 \\
\hline $10 \mathrm{H}-5,83-85$ & 89.81 & OT & $\mathrm{AF}$ & $6.24 \mathrm{E}-04$ & & & & 4 \\
\hline $10 \mathrm{H}-6,89-91$ & 91.37 & $\mathrm{RM}$ & $\mathrm{AF}$ & $3.63 \mathrm{E}-04$ & -59.55 & 23.0 & 17-or & 3 \\
\hline $10 \mathrm{H}-7,84-86$ & 92.82 & $\mathrm{RM}$ & $\mathrm{AF}$ & $4.75 \mathrm{E}-04$ & -8.37 & 21.8 & 17-or & 3 \\
\hline $10 \mathrm{H}-8,43-45$ & 93.41 & $\mathrm{RM}$ & $\mathrm{AF}$ & $4.86 \mathrm{E}-04$ & & & & 4 \\
\hline $11 \mathrm{H}-2,52-54$ & 95.74 & $\mathrm{RM}$ & $\mathrm{AF}$ & $5.62 \mathrm{E}-04$ & & & & 4 \\
\hline $11 \mathrm{H}-3,57-59$ & 97.29 & $\mathrm{RM}$ & $\mathrm{AF}$ & $4.65 \mathrm{E}-04$ & -11.88 & 13.2 & 13-or & 3 \\
\hline $11 \mathrm{H}-4,134-136$ & 99.54 & OT & $\mathrm{AF}$ & $7.46 \mathrm{E}-04$ & -74.20 & 10.1 & 15 -or & 3 \\
\hline $11 \mathrm{H}-5,115-117$ & 100.85 & $\mathrm{RM}$ & $\mathrm{AF}$ & $7.48 \mathrm{E}-04$ & 15.28 & 9.2 & $45-80$ & 3 \\
\hline $11 \mathrm{H}-6,44-46$ & 101.64 & $\mathrm{RM}$ & $\mathrm{AF}$ & $9.07 \mathrm{E}-04$ & -31.92 & 13.6 & 30 -or & 2 \\
\hline $12 \mathrm{H}-4,86-88$ & 108.54 & $\mathrm{RM}$ & $\mathrm{AF}$ & $2.83 \mathrm{E}-04$ & -7.24 & 30.5 & $17-40$ & 3 \\
\hline $12 \mathrm{H}-5,86-88$ & 110.04 & $\mathrm{RM}$ & $\mathrm{AF}$ & $4.54 \mathrm{E}-04$ & -51.47 & 9.7 & 21 -or & 1 \\
\hline $12 \mathrm{H}-6,126-128$ & 111.94 & $\mathrm{RM}$ & $\mathrm{AF}$ & $5.23 \mathrm{E}-04$ & -53.45 & 11.1 & 17-or & 2 \\
\hline $13 \mathrm{H}-1,133-135$ & 114.05 & $\mathrm{RM}$ & $\mathrm{AF}$ & $6.53 \mathrm{E}-04$ & -35.20 & 12.9 & 25 -or & 2 \\
\hline $13 \mathrm{H}-3,71-73$ & 116.41 & $\mathrm{RM}$ & $\mathrm{AF}$ & $3.58 \mathrm{E}-04$ & & & & 4 \\
\hline $13 \mathrm{H}-4,112-114$ & 118.32 & OT & $\mathrm{AF}$ & 4.03E-04 & -38.60 & 5.2 & 10-or & 3 \\
\hline $14 \mathrm{H}-2,85-87$ & 124.57 & $\mathrm{RM}$ & $\mathrm{AF}$ & $7.48 \mathrm{E}-04$ & -68.24 & 12.8 & 25 -or & 1 \\
\hline $14 \mathrm{H}-3,129-131$ & 126.51 & OT & $\mathrm{AF}$ & $9.63 \mathrm{E}-04$ & -53.60 & 23.5 & 40 -or & 2 \\
\hline $14 \mathrm{H}-4,47-49$ & 127.19 & $\mathrm{RM}$ & $\mathrm{AF}$ & $4.30 \mathrm{E}-04$ & -68.83 & 10.9 & 21 -or & 2 \\
\hline $14 \mathrm{H}-5,78-80$ & 129.00 & RM & $\mathrm{AF}$ & $3.41 \mathrm{E}-04$ & -57.01 & 6.3 & 17-or & 3 \\
\hline $15 \mathrm{H}-5,51-53$ & 138.03 & $\mathrm{RM}$ & $\mathrm{AF}$ & $1.94 \mathrm{E}-03$ & 53.25 & 14.1 & 30 -or & 2 \\
\hline $15 \mathrm{H}-6,86-88$ & 139.88 & $\mathrm{RM}$ & $\mathrm{AF}$ & $3.09 \mathrm{E}-04$ & -40.66 & 13.7 & 30 -or & 2 \\
\hline $16 \mathrm{H}-1,44-46$ & 141.66 & $\mathrm{RM}$ & $\mathrm{AF}$ & $6.79 \mathrm{E}-04$ & 51.47 & 20.1 & 25 -or & 3 \\
\hline $16 \mathrm{H}-4,52-54$ & 146.21 & OT & $\mathrm{AF}$ & $6.62 \mathrm{E}-04$ & & & & 4 \\
\hline $17 \mathrm{H}-4,73-75$ & 155.24 & $\mathrm{RM}$ & $\mathrm{AF}$ & $3.94 \mathrm{E}-04$ & 3.08 & 18.8 & 21 -or & 2 \\
\hline $18 \mathrm{H}-1,99-101$ & 157.21 & $\mathrm{RM}$ & $\mathrm{AF}$ & $4.46 \mathrm{E}-04$ & 50.26 & 13.2 & 45 -or & 1 \\
\hline $18 \mathrm{H}-3,66-68$ & 159.88 & OT & $\mathrm{AF}$ & $5.46 \mathrm{E}-04$ & -40.20 & 11.3 & 25 -or & 2 \\
\hline $18 \mathrm{H}-5,86-88$ & 161.48 & RM & $\mathrm{AF}$ & $1.90 \mathrm{E}-04$ & 57.81 & 11.1 & 45 -or & 1 \\
\hline $18 \mathrm{H}-6,54-56$ & 162.66 & $\mathrm{RM}$ & $\mathrm{AF}$ & $1.99 \mathrm{E}-04$ & 51.52 & 7.3 & $40-60$ & 1 \\
\hline $19 \mathrm{H}-2,39-41$ & 167.61 & RM & $\mathrm{AF}$ & $6.38 \mathrm{E}-04$ & -55.61 & 0.5 & 21 -or & 1 \\
\hline $19 \mathrm{H}-3,41-43$ & 169.13 & $\mathrm{RM}$ & $\mathrm{AF}$ & $1.38 \mathrm{E}-04$ & -72.60 & 4.7 & 17-or & 2 \\
\hline $19 \mathrm{H}-5,38-40$ & 172.10 & $\mathrm{RM}$ & $\mathrm{AF}$ & $9.06 \mathrm{E}-05$ & -16.58 & 16.8 & 25 -or & 3 \\
\hline $20 \mathrm{H}-1,126-128$ & 175.98 & OT & $\mathrm{AF}$ & $4.94 \mathrm{E}-04$ & -48.80 & 8.3 & 25 -or & 1 \\
\hline $20 \mathrm{H}-2,85-87$ & 177.07 & $\mathrm{RM}$ & $\mathrm{AF}$ & $3.21 \mathrm{E}-04$ & & & & 4 \\
\hline $21 \mathrm{H}-2,78-80$ & 182.00 & $\mathrm{RM}$ & $\mathrm{AF}$ & $4.04 \mathrm{E}-04$ & -36.52 & 17.3 & 13 -or & 3 \\
\hline $21 \mathrm{H}-6,25-27$ & 187.47 & OT & $\mathrm{AF}$ & $6.47 \mathrm{E}-04$ & & & & 4 \\
\hline
\end{tabular}


Table T2 (continued). (Continued on next page.)

\begin{tabular}{|c|c|c|c|c|c|c|c|c|}
\hline $\begin{array}{l}\text { Core, section, } \\
\text { interval }(\mathrm{cm})\end{array}$ & $\begin{array}{c}\text { Depth } \\
\text { CSF-A (m) }\end{array}$ & Lab & Treatment & $\begin{array}{c}\text { NRM } \\
\text { intensity } \\
(\mathrm{A} / \mathrm{m})\end{array}$ & $\begin{array}{c}\text { ChRM } \\
\text { inclination } \\
\left(^{\circ}\right)\end{array}$ & $\begin{array}{c}\text { MAD } \\
\left({ }^{\circ}\right)\end{array}$ & Steps & Class \\
\hline $22 \mathrm{H}-1,80-82$ & 190.02 & RM & $\mathrm{AF}$ & $9.63 \mathrm{E}-04$ & -67.19 & 4.4 & 17-or & 2 \\
\hline $22 \mathrm{H}-2,84-86$ & 191.56 & RM & $\mathrm{AF}$ & 2.77E-03 & & & & 4 \\
\hline $22 \mathrm{H}-3,96-98$ & 193.18 & RM & $\mathrm{AF}$ & $1.17 \mathrm{E}-03$ & -34.01 & 17.1 & 17-or & 3 \\
\hline $22 \mathrm{H}-4,110-112$ & 194.82 & От & $\mathrm{AF}$ & $1.98 \mathrm{E}-03$ & -68.60 & 8.3 & 30 -or & 2 \\
\hline $22 \mathrm{H}-5,92-94$ & 196.14 & RM & $\mathrm{AF}$ & $7.38 \mathrm{E}-04$ & -33.33 & 31.0 & 21 -or & 2 \\
\hline $23 \mathrm{H}-1,53-55$ & 199.25 & RM & $\mathrm{AF}$ & $7.60 \mathrm{E}-04$ & -52.62 & 30.3 & 13-or & 3 \\
\hline $23 \mathrm{H}-4,87-89$ & 204.09 & RM & $\mathrm{AF}$ & 7.70E-04 & & & & 4 \\
\hline 23H-5, 109-111 & 205.81 & RM & $\mathrm{AF}$ & 1.77E-04 & -51.57 & 13.9 & 13-or & 2 \\
\hline $24 \mathrm{H}-1,98-100$ & 209.20 & RM & $\mathrm{AF}$ & $7.98 \mathrm{E}-04$ & -62.67 & 13.4 & 17-or & 2 \\
\hline $24 \mathrm{H}-3,73-75$ & 211.95 & RM & $\mathrm{AF}$ & $6.18 \mathrm{E}-04$ & -24.47 & 12.1 & 21 -or & 3 \\
\hline $24 \mathrm{H}-4,91-93$ & 213.63 & OT & $\mathrm{AF}$ & $3.99 \mathrm{E}-04$ & & & & 4 \\
\hline $24 \mathrm{H}-5,85-87$ & 215.07 & RM & $\mathrm{AF}$ & $3.04 \mathrm{E}-04$ & -55.66 & 23.3 & 17-or & 2 \\
\hline $24 \mathrm{H}-6,58-60$ & 216.30 & RM & $\mathrm{AF}$ & $3.15 \mathrm{E}-04$ & & & & 4 \\
\hline $24 \mathrm{H}-7,37-39$ & 217.59 & RM & $\mathrm{AF}$ & $2.93 \mathrm{E}-04$ & -11.75 & 28.7 & 17-or & 3 \\
\hline $25 \mathrm{H}-1,77-79$ & 218.49 & RM & $\mathrm{AF}$ & $3.46 \mathrm{E}-04$ & -20.72 & 17.4 & 25 -or & 3 \\
\hline $25 \mathrm{H}-2,102-104$ & 220.24 & RM & $\mathrm{AF}$ & $9.12 \mathrm{E}-05$ & -66.41 & 21.4 & 13-or & 3 \\
\hline $25 \mathrm{H}-4,72-74$ & 222.94 & RM & $\mathrm{AF}$ & $2.08 \mathrm{E}-04$ & -9.51 & 18.6 & 21 -or & 2 \\
\hline $25 \mathrm{H}-5,49-51$ & 224.21 & RM & $\mathrm{AF}$ & $1.80 \mathrm{E}-04$ & -33.09 & 27.3 & 30 -or & 1 \\
\hline $26 \mathrm{H}-1,86-81$ & 228.01 & RM & $\mathrm{AF}$ & $3.53 \mathrm{E}-04$ & -35.03 & 10.3 & 40 -or & 2 \\
\hline $26 \mathrm{H}-4,82-84$ & 232.46 & RM & $\mathrm{AF}$ & $1.82 \mathrm{E}-04$ & -54.93 & 3.0 & 50 -or & 2 \\
\hline $26 \mathrm{H}-5,78-80$ & 233.92 & RM & $\mathrm{AF}$ & $1.74 \mathrm{E}-04$ & -54.47 & 10.3 & 17-or & 2 \\
\hline $26 \mathrm{H}-6,49-51$ & 235.13 & RM & $\mathrm{AF}$ & $8.36 \mathrm{E}-04$ & -61.49 & 7.0 & 35 -or & 2 \\
\hline $27 \mathrm{H}-1,48-50$ & 237.20 & RM & $\mathrm{AF}$ & $6.00 \mathrm{E}-04$ & -64.75 & 9.2 & 35 -or & 2 \\
\hline $27 \mathrm{H}-4,87-89$ & 242.09 & RM & $\mathrm{AF}$ & $3.48 \mathrm{E}-04$ & -21.93 & 16.4 & 25 -or & 2 \\
\hline $28 \mathrm{H}-2,90-92$ & 248.55 & OT & $\mathrm{AF}$ & $5.04 \mathrm{E}-04$ & -68.20 & 20.7 & 40 -or & 3 \\
\hline $28 \mathrm{H}-4,35-37$ & 250.50 & RM & $\mathrm{AF}$ & $5.29 \mathrm{E}-04$ & -28.88 & 15.9 & 21 -or & 3 \\
\hline $29 \mathrm{H}-2,78-80$ & 253.50 & RM & $\mathrm{AF}$ & $3.07 \mathrm{E}-03$ & 54.81 & 14.4 & 30 -or & 2 \\
\hline $30 \mathrm{H}-1,57-59$ & 258.29 & RM & $\mathrm{AF}$ & $1.24 \mathrm{E}-03$ & 11.91 & 21.4 & $21-60$ & 3 \\
\hline $30 \mathrm{H}-2,46-48$ & 259.68 & OT & $\mathrm{AF}$ & $9.75 \mathrm{E}-04$ & & & & 4 \\
\hline $30 \mathrm{H}-4,39-41$ & 262.61 & RM & $\mathrm{AF}$ & $1.86 \mathrm{E}-03$ & 41.70 & 12.6 & 30 -or & 1 \\
\hline $31 \mathrm{H}-2,35-37$ & 269.07 & RM & $\mathrm{AF}$ & $4.82 \mathrm{E}-03$ & 41.62 & 3.6 & 30 -or & 1 \\
\hline $31 \mathrm{H}-5,31-33$ & 271.34 & От & $\mathrm{AF}$ & $3.08 \mathrm{E}-03$ & & & & 4 \\
\hline $32 \mathrm{H}-2,51-53$ & 274.23 & От & $\mathrm{AF}$ & $1.64 \mathrm{E}-03$ & 46.50 & 20.6 & 25 -or & 3 \\
\hline $32 \mathrm{H}-3,52-54$ & 275.74 & OT & $\mathrm{AF}$ & $7.09 \mathrm{E}-04$ & 35.80 & 9.8 & 30 -or & 2 \\
\hline $32 \mathrm{H}-3,55-57$ & 275.77 & RM & $\mathrm{AF}$ & $6.26 \mathrm{E}-04$ & & & & 4 \\
\hline $33 \mathrm{H}-6,42-44$ & 288.06 & RM & $\mathrm{AF}$ & $7.48 \mathrm{E}-04$ & & & & 4 \\
\hline $36 \mathrm{H}-1,48-50$ & 295.80 & OT & $\mathrm{AF}$ & $6.58 \mathrm{E}-04$ & -29.50 & 14.6 & 25 -or & 2 \\
\hline $38 X-1,98-100$ & 303.60 & RM & $\mathrm{AF}$ & $4.05 \mathrm{E}-04$ & & & & 4 \\
\hline $38 X-2,101-103$ & 305.13 & RM & $\mathrm{AF}$ & $2.01 \mathrm{E}-04$ & -19.81 & 3.8 & 25 -or & 3 \\
\hline $38 X-5,116-118$ & 309.78 & RM & $\mathrm{AF}$ & $3.90 \mathrm{E}-04$ & 20.66 & 11.3 & 30 -or & 2 \\
\hline $39 X-1,75-77$ & 312.97 & RM & $\mathrm{AF}$ & $4.93 \mathrm{E}-04$ & 56.58 & 3.2 & 30 -or & 1 \\
\hline $39 X-2,75-77$ & 314.47 & RM & $\mathrm{AF}$ & $2.40 \mathrm{E}-05$ & & & & 4 \\
\hline $39 X-3,69-71$ & 315.91 & RM & $\mathrm{AF}$ & $1.98 \mathrm{E}-04$ & -69.97 & 13.1 & 21 -or & 1 \\
\hline $39 X-4,99-101$ & 317.71 & RM & $\mathrm{AF}$ & $1.17 \mathrm{E}-04$ & -7.38 & 13.5 & 13-or & 3 \\
\hline $39 X-5,129-131$ & 319.51 & От & $\mathrm{AF}$ & $3.58 \mathrm{E}-04$ & -24.90 & 7.9 & 50 -or & 2 \\
\hline $39 X-6,22-24$ & 319.94 & RM & $\mathrm{AF}$ & $7.52 \mathrm{E}-05$ & & & & 4 \\
\hline $39 X-7,42-44$ & 321.44 & RM & $\mathrm{AF}$ & 4.79E-04 & 62.86 & 26.1 & 21 -or & 3 \\
\hline $40 X-1,91-93$ & 322.83 & RM & $\mathrm{AF}$ & $4.33 \mathrm{E}-04$ & -69.90 & 14.1 & 25 -or & 3 \\
\hline $40 \times-2,68-70$ & 324.10 & RM & $\mathrm{AF}$ & $1.19 \mathrm{E}-04$ & 9.25 & 14.2 & 21 -or & 3 \\
\hline $40 X-3,86-88$ & 325.78 & RM & $\mathrm{AF}$ & $2.83 \mathrm{E}-04$ & & & & 4 \\
\hline $40 X-5,51-53$ & 328.43 & RM & $\mathrm{AF}$ & $8.43 \mathrm{E}-04$ & 48.29 & 9.9 & 40 -or & 2 \\
\hline $40 X-6,19-21$ & 329.61 & RM & $\mathrm{AF}$ & $1.92 \mathrm{E}-04$ & & & & 4 \\
\hline $41 X-1,117-119$ & 332.69 & RM & $\mathrm{AF}$ & $1.69 \mathrm{E}-04$ & -39.33 & 16.6 & 30 -or & 3 \\
\hline $41 X-2,86-88$ & 333.88 & RM & $\mathrm{AF}$ & $5.29 \mathrm{E}-04$ & 36.13 & 25.2 & 21 -or & 3 \\
\hline $41 X-3,71-73$ & 335.23 & RM & $\mathrm{AF}$ & $3.78 \mathrm{E}-04$ & & & & 4 \\
\hline $41 X-4,89-91$ & 336.91 & От & $\mathrm{AF}$ & $6.11 \mathrm{E}-04$ & & & & 4 \\
\hline $41 X-5,75-77$ & 338.27 & RM & $\mathrm{AF}$ & $2.89 \mathrm{E}-04$ & -20.45 & 11.9 & 30 -or & 3 \\
\hline $41 X-6,72-74$ & 339.74 & RM & $\mathrm{AF}$ & $4.76 \mathrm{E}-04$ & -17.87 & 20.9 & 17-or & 3 \\
\hline $41 X-7,51-53$ & 340.63 & RM & $\mathrm{AF}$ & $2.88 \mathrm{E}-04$ & -22.91 & 27.0 & 17-or & 3 \\
\hline $42 X-1,69-70$ & 341.80 & RM & $\mathrm{AF}$ & $2.12 \mathrm{E}-04$ & 21.12 & 13.4 & 25 -or & 3 \\
\hline $42 X-2,90-92$ & 343.52 & OT & $\mathrm{AF}$ & $1.40 \mathrm{E}-04$ & & & & 4 \\
\hline $42 X-3,81-83$ & 344.93 & RM & $\mathrm{AF}$ & $2.47 \mathrm{E}-04$ & & & & 4 \\
\hline $42 X-4,73-75$ & 346.35 & RM & $\mathrm{AF}$ & $1.62 \mathrm{E}-04$ & & & & 4 \\
\hline $43 X-1,101-103$ & 351.73 & RM & $\mathrm{AF}$ & $9.59 \mathrm{E}-05$ & -20.44 & 7.5 & $25-35$ & 3 \\
\hline $43 X-2,128-130$ & 353.50 & RM & $\mathrm{AF}$ & $2.59 \mathrm{E}-04$ & -27.65 & 13.7 & 35 -or & 3 \\
\hline $43 X-3,130-132$ & 355.02 & RM & $\mathrm{AF}$ & $2.88 \mathrm{E}-04$ & -7.32 & 20.8 & 40 -or & 3 \\
\hline $43 X-4,77-79$ & 355.99 & OT & $\mathrm{AF}$ & $4.55 \mathrm{E}-04$ & -50.40 & 20.1 & 45 -or & 2 \\
\hline $43 X-5,78-80$ & 357.50 & RM & $\mathrm{AF}$ & $1.60 \mathrm{E}-04$ & & & & 4 \\
\hline
\end{tabular}


Table T2 (continued). (Continued on next page.)

\begin{tabular}{|c|c|c|c|c|c|c|c|c|}
\hline $\begin{array}{l}\text { Core, section, } \\
\text { interval }(\mathrm{cm})\end{array}$ & $\begin{array}{c}\text { Depth } \\
\text { CSF-A (m) }\end{array}$ & Lab & Treatment & $\begin{array}{c}\text { NRM } \\
\text { intensity } \\
(\mathrm{A} / \mathrm{m})\end{array}$ & $\begin{array}{c}\text { ChRM } \\
\text { inclination } \\
\left({ }^{\circ}\right)\end{array}$ & $\begin{array}{c}\text { MAD } \\
\left({ }^{\circ}\right)\end{array}$ & Steps & Class \\
\hline $43 X-6,62-64$ & 358.84 & $\mathrm{RM}$ & $\mathrm{AF}$ & $9.85 \mathrm{E}-05$ & & & & 4 \\
\hline $44 X-1,88-90$ & 361.30 & $\mathrm{RM}$ & $\mathrm{AF}$ & $1.04 \mathrm{E}-03$ & 46.64 & 15.5 & 17-or & 2 \\
\hline $44 X-2,102-104$ & 362.94 & $\mathrm{RM}$ & $\mathrm{AF}$ & $9.00 \mathrm{E}-04$ & 37.60 & 16.2 & 35 -or & 3 \\
\hline $44 X-3,91-93$ & 364.33 & OT & $\mathrm{AF}$ & $1.06 \mathrm{E}-03$ & & & & 4 \\
\hline $44 X-4,81-83$ & 365.73 & $\mathrm{RM}$ & $\mathrm{AF}$ & $6.57 \mathrm{E}-04$ & 60.00 & 26.0 & 50 -or & 3 \\
\hline $44 X-5,79-81$ & 367.21 & $\mathrm{RM}$ & $\mathrm{AF}$ & $6.26 \mathrm{E}-04$ & 69.41 & 12.1 & 45 -or & 2 \\
\hline $44 X-6,71-73$ & 368.63 & $\mathrm{RM}$ & $\mathrm{AF}$ & $6.55 \mathrm{E}-04$ & 80.64 & 18.1 & 21 -or & 3 \\
\hline $44 X-6,71-73$ & 368.63 & $\mathrm{RM}$ & $\mathrm{AF}$ & $5.61 \mathrm{E}-05$ & 80.64 & 18.1 & 21 -or & 3 \\
\hline $45 X-1,86-88$ & 370.88 & $\mathrm{RM}$ & $\mathrm{AF}$ & $1.25 \mathrm{E}-04$ & -40.78 & 19.1 & 30 -or & 2 \\
\hline $45 X-2,73-75$ & 372.25 & $\mathrm{RM}$ & $\mathrm{AF}$ & $1.22 \mathrm{E}-04$ & & & & 4 \\
\hline $45 X-3,89-91$ & 373.91 & OT & $\mathrm{AF}$ & $3.36 \mathrm{E}-04$ & & & & 4 \\
\hline $45 X-5,84-86$ & 376.86 & $\mathrm{RM}$ & $\mathrm{AF}$ & $2.06 \mathrm{E}-04$ & -12.27 & 6.5 & 17-or & 3 \\
\hline $45 X-6,42-44$ & 377.94 & $\mathrm{RM}$ & $\mathrm{AF}$ & $3.17 \mathrm{E}-04$ & 37.14 & 18.5 & 25 -or & 2 \\
\hline $46 X-1,83-85$ & 380.45 & $\mathrm{RM}$ & $\mathrm{AF}$ & $1.24 \mathrm{E}-03$ & 34.18 & 8.9 & 21-or & 3 \\
\hline $46 X-2,83-85$ & 381.95 & $\mathrm{RM}$ & $\mathrm{AF}$ & $1.17 \mathrm{E}-03$ & & & & 4 \\
\hline $46 X-3,83-85$ & 383.43 & $\mathrm{RM}$ & $\mathrm{AF}$ & $6.19 \mathrm{E}-04$ & -17.70 & 17.7 & 45 -or & 2 \\
\hline $46 X-4,83-85$ & 384.93 & $\mathrm{RM}$ & $\mathrm{AF}$ & $4.29 \mathrm{E}-04$ & -29.91 & 15.0 & 35 -or & 3 \\
\hline $46 X-5,83-85$ & 386.43 & $\mathrm{RM}$ & $\mathrm{AF}$ & $9.59 \mathrm{E}-04$ & -75.26 & 30.6 & 35 -or & 3 \\
\hline $46 X-6,83-85$ & 387.93 & OT & $\mathrm{AF}$ & $2.37 \mathrm{E}-03$ & & & & 4 \\
\hline $47 X-1,47-49$ & 389.69 & $\mathrm{RM}$ & $\mathrm{AF}$ & $4.32 \mathrm{E}-04$ & & & & 4 \\
\hline $47 X-3,50-52$ & 392.72 & $\mathrm{RM}$ & $\mathrm{AF}$ & $1.46 \mathrm{E}-04$ & -27.04 & 13.7 & 45 -or & 2 \\
\hline $47 X-4,46-48$ & 394.18 & $\mathrm{RM}$ & $\mathrm{AF}$ & $3.17 \mathrm{E}-04$ & 10.90 & 24.0 & 30 -or & 3 \\
\hline $47 X-5,34-36$ & 395.56 & $\mathrm{RM}$ & $\mathrm{AF}$ & $5.62 \mathrm{E}-04$ & -40.06 & 30.0 & 40 -or & 3 \\
\hline $47 X-6,34-36$ & 397.06 & OT & $\mathrm{AF}$ & $1.88 \mathrm{E}-03$ & & & & 4 \\
\hline $47 X-7,23-25$ & 398.25 & $\mathrm{RM}$ & $\mathrm{AF}$ & $1.12 \mathrm{E}-03$ & -23.08 & 16.0 & 50 -or & 3 \\
\hline $48 X-1,90-92$ & 399.62 & $\mathrm{RM}$ & $\mathrm{AF}$ & $6.06 \mathrm{E}-04$ & 61.35 & 10.5 & 25 -or & 2 \\
\hline $48 X-2,90-92$ & 401.12 & $\mathrm{RM}$ & $\mathrm{AF}$ & $6.46 \mathrm{E}-04$ & 26.50 & 19.8 & 21 -or & 3 \\
\hline $48 X-3,88-90$ & 402.60 & $\mathrm{RM}$ & $\mathrm{AF}$ & $4.69 \mathrm{E}-04$ & 71.16 & 26.9 & 45 -or & 3 \\
\hline $48 X-4,85-87$ & 404.07 & $\mathrm{RM}$ & $\mathrm{AF}$ & $6.15 \mathrm{E}-04$ & 41.09 & 20.2 & 21 -or & 3 \\
\hline $48 X-5,85-87$ & 405.57 & $\mathrm{RM}$ & $\mathrm{AF}$ & $2.93 \mathrm{E}-04$ & & & & 4 \\
\hline $48 X-6,74-76$ & 406.96 & OT & $\mathrm{AF}$ & $1.99 \mathrm{E}-03$ & & & & 4 \\
\hline $49 X-2,112-114$ & 410.94 & OT & $\mathrm{AF}$ & $6.10 \mathrm{E}-04$ & & & & 4 \\
\hline $49 X-3,52-54$ & 411.84 & $\mathrm{RM}$ & $\mathrm{AF}$ & $3.68 \mathrm{E}-04$ & -50.49 & 22.6 & 25 -or & 3 \\
\hline $50 X-1,52-54$ & 418.44 & $\mathrm{RM}$ & $\mathrm{AF}$ & $7.03 \mathrm{E}-05$ & 35.97 & 17.7 & 30 -or & 2 \\
\hline $50 x-2,117-119$ & 420.59 & $\mathrm{RM}$ & $\mathrm{AF}$ & $5.86 \mathrm{E}-05$ & 26.83 & 14.4 & 25 -or & 2 \\
\hline $50 X-3,50-52$ & 421.42 & $\mathrm{RM}$ & $\mathrm{AF}$ & $9.48 \mathrm{E}-05$ & -16.67 & 9.5 & 25 -or & 3 \\
\hline $50 \times-4,50-52$ & 422.92 & $\mathrm{RM}$ & $\mathrm{AF}$ & $2.76 \mathrm{E}-04$ & 12.05 & 17.7 & 30-or & 2 \\
\hline $50 X-5,102-104$ & 424.94 & $\mathrm{RM}$ & $\mathrm{AF}$ & $3.56 \mathrm{E}-04$ & 17.50 & 11.3 & 21 -or & 2 \\
\hline $50 x-6,98-100$ & 426.40 & OT & $\mathrm{AF}$ & $6.00 \mathrm{E}-04$ & & & & 4 \\
\hline $50 X-7,40-42$ & 427.12 & $\mathrm{RM}$ & $\mathrm{AF}$ & $2.73 \mathrm{E}-04$ & 46.53 & 4.0 & 17-or & 1 \\
\hline $51 X-1,85-87$ & 428.37 & $\mathrm{RM}$ & $\mathrm{AF}$ & $3.33 \mathrm{E}-04$ & 61.23 & 13.7 & 25 -or & 3 \\
\hline $51 X-2,88-90$ & 429.90 & $\mathrm{RM}$ & $\mathrm{AF}$ & $1.73 \mathrm{E}-04$ & -22.61 & 26.6 & 25 -or & 3 \\
\hline $51 X-3,92-94$ & 431.44 & $\mathrm{RM}$ & $\mathrm{AF}$ & $1.75 \mathrm{E}-04$ & & & & 4 \\
\hline $51 X-4,110-112$ & 433.12 & $\mathrm{RM}$ & $\mathrm{AF}$ & $2.33 \mathrm{E}-04$ & -49.70 & 16.5 & 35 -or & 2 \\
\hline $51 X-5,80-82$ & 434.32 & OT & $\mathrm{AF}$ & $5.56 \mathrm{E}-04$ & & & & 4 \\
\hline $51 X-6,88-90$ & 435.90 & $\mathrm{RM}$ & $\mathrm{AF}$ & $4.43 \mathrm{E}-04$ & -51.79 & 33.5 & 25 -or & 3 \\
\hline $51 X-7,51-53$ & 436.73 & $\mathrm{RM}$ & $\mathrm{AF}$ & $3.02 \mathrm{E}-04$ & -52.93 & 7.3 & 21 -or & 3 \\
\hline $52 X-1,53-55$ & 437.65 & $\mathrm{RM}$ & $\mathrm{AF}$ & $7.82 \mathrm{E}-05$ & 63.79 & 14.2 & 35 -or & 1 \\
\hline $52 X-2,78-80$ & 439.40 & $\mathrm{RM}$ & $\mathrm{AF}$ & $1.12 \mathrm{E}-04$ & 24.45 & 15.1 & 35 -or & 2 \\
\hline $52 X-3,109-111$ & 441.21 & $\mathrm{RM}$ & $\mathrm{AF}$ & $1.32 \mathrm{E}-04$ & -7.63 & 12.4 & 40 -or & 3 \\
\hline $52 X-4,77-79$ & 442.39 & OT & $\mathrm{AF}$ & $5.98 \mathrm{E}-05$ & & & & 4 \\
\hline $52 X-5,71-73$ & 443.83 & $\mathrm{RM}$ & $\mathrm{AF}$ & $3.30 \mathrm{E}-05$ & -7.71 & 13.8 & 50 -or & 3 \\
\hline $52 X-6,74-76$ & 445.36 & $\mathrm{RM}$ & $\mathrm{AF}$ & $6.28 \mathrm{E}-05$ & -2.06 & 12.8 & 25 -or & 3 \\
\hline $52 X-7,19-21$ & 446.01 & $\mathrm{RM}$ & $\mathrm{AF}$ & $1.02 \mathrm{E}-04$ & 55.44 & 17.5 & 21 -or & 2 \\
\hline $53 X-5,89-91$ & 453.61 & OT & $\mathrm{AF}$ & $2.08 \mathrm{E}-04$ & & & & 4 \\
\hline $53 X-6,89-91$ & 455.08 & $\mathrm{RM}$ & $\mathrm{AF}$ & $5.03 \mathrm{E}-04$ & 43.32 & 25.9 & 25 -or & 3 \\
\hline $54 X-1,81-83$ & 457.13 & $\mathrm{RM}$ & $\mathrm{AF}$ & $2.60 \mathrm{E}-05$ & & & & 4 \\
\hline $54 X-2,70-72$ & 458.52 & $\mathrm{RM}$ & $\mathrm{AF}$ & $2.99 \mathrm{E}-05$ & & & & 4 \\
\hline $54 X-5,110-112$ & 463.42 & $\mathrm{RM}$ & $\mathrm{AF}$ & $6.41 \mathrm{E}-05$ & 45.61 & 21.7 & 21 -or & 3 \\
\hline $55 X-2,138-140$ & 468.80 & OT & $\mathrm{AF}$ & $1.75 \mathrm{E}-04$ & & & & 4 \\
\hline $55 X-3,33-35$ & 469.25 & $\mathrm{RM}$ & $\mathrm{AF}$ & $5.52 \mathrm{E}-05$ & -15.83 & 9.6 & 25 -or & 2 \\
\hline $56 X-1,109-111$ & 476.61 & $\mathrm{RM}$ & $\mathrm{AF}$ & $3.64 \mathrm{E}-05$ & -32.56 & 25.6 & 30 -or & 3 \\
\hline $56 X-2,85-87$ & 477.87 & OT & $\mathrm{AF}$ & $2.43 \mathrm{E}-04$ & & & & 4 \\
\hline $56 X-6,41-43$ & 483.43 & $\mathrm{RM}$ & $\mathrm{AF}$ & $7.90 \mathrm{E}-05$ & -21.46 & 15.9 & 40 -or & 3 \\
\hline $57 X-1,117-120$ & 486.30 & $\mathrm{RM}$ & $\mathrm{AF}$ & $5.23 \mathrm{E}-05$ & & & & 4 \\
\hline $57 X-2,82-84$ & 487.44 & OT & $\mathrm{AF}$ & $7.38 \mathrm{E}-05$ & & & & 4 \\
\hline $57 X-3,81-83$ & 488.93 & $\mathrm{RM}$ & $\mathrm{AF}$ & $2.71 \mathrm{E}-05$ & -35.22 & 31.4 & 30 -or & 2 \\
\hline $58 X-2,90-92$ & 495.84 & $\mathrm{RM}$ & $\mathrm{AF}$ & $2.43 \mathrm{E}-05$ & -51.91 & 19.0 & 21 -or & 3 \\
\hline
\end{tabular}


Table T2 (continued). (Continued on next page.)

\begin{tabular}{|c|c|c|c|c|c|c|c|c|}
\hline $\begin{array}{l}\text { Core, section, } \\
\text { interval }(\mathrm{cm})\end{array}$ & $\begin{array}{c}\text { Depth } \\
\text { CSF-A (m) }\end{array}$ & Lab & Treatment & $\begin{array}{c}\text { NRM } \\
\text { intensity } \\
(\mathrm{A} / \mathrm{m})\end{array}$ & $\begin{array}{c}\text { ChRM } \\
\text { inclination } \\
\left({ }^{\circ}\right)\end{array}$ & $\begin{array}{c}\text { MAD } \\
\left({ }^{\circ}\right)\end{array}$ & Steps & Class \\
\hline $58 X-3,90-92$ & 497.34 & RM & $\mathrm{AF}$ & $2.42 \mathrm{E}-04$ & -26.38 & 20.2 & 45 -or & 2 \\
\hline $58 X-4,90-92$ & 498.84 & RM & $\mathrm{AF}$ & $4.81 \mathrm{E}-04$ & -36.02 & 9.5 & 50 -or & 3 \\
\hline $58 X-5,94-96$ & 500.38 & OT & $\mathrm{AF}$ & $3.41 \mathrm{E}-04$ & & & & 4 \\
\hline $58 X-6,90-92$ & 501.84 & RM & $\mathrm{AF}$ & $2.30 \mathrm{E}-04$ & & & & 4 \\
\hline $58 X-7,90-92$ & 503.34 & RM & $\mathrm{AF}$ & $2.40 \mathrm{E}-04$ & -62.99 & 23.6 & 35 -or & 3 \\
\hline $59 X-1,90-92$ & 505.22 & RM & $\mathrm{AF}$ & $2.28 \mathrm{E}-04$ & 9.94 & 21.8 & 21 -or & 3 \\
\hline $59 X-1,94-96$ & 505.26 & OT & $\mathrm{AF}$ & $6.61 \mathrm{E}-04$ & & & & 4 \\
\hline $61 X-1,53-55$ & 524.05 & OT & $\mathrm{AF}$ & $1.96 \mathrm{E}-04$ & -18.80 & 11.5 & 25 -or & 2 \\
\hline $61 X-2,17-19$ & 524.69 & RM & $\mathrm{AF}$ & $6.64 \mathrm{E}-05$ & 55.38 & 13.8 & 25 -or & 2 \\
\hline $63 X-2,114-117$ & 545.37 & RM & $\mathrm{AF}$ & $2.02 \mathrm{E}-04$ & 36.96 & 16.2 & 35 -or & 1 \\
\hline $63 X-3,116-118$ & 546.88 & RM & $\mathrm{AF}$ & $2.95 \mathrm{E}-04$ & 23.40 & 11.1 & 30 -or & 1 \\
\hline $63 X-4,110-112$ & 548.32 & RM & $\mathrm{AF}$ & $1.44 \mathrm{E}-04$ & 33.13 & 27.9 & 21 -or & 3 \\
\hline $63 X-5,118-120$ & 549.90 & От & $\mathrm{AF}$ & $1.61 \mathrm{E}-03$ & & & & 4 \\
\hline $63 X-6,46-48$ & 550.68 & RM & $\mathrm{AF}$ & $2.05 \mathrm{E}-04$ & 49.88 & 16.0 & 30 -or & 2 \\
\hline $63 X-7,38-40$ & 552.00 & RM & $\mathrm{AF}$ & $2.91 \mathrm{E}-04$ & 48.50 & 13.1 & 35 -or & 2 \\
\hline $64 X-1,114-116$ & 553.36 & RM & $\mathrm{AF}$ & $6.24 \mathrm{E}-05$ & 56.21 & 27.8 & 30 -or & 3 \\
\hline $64 X-2,52-54$ & 554.24 & RM & $\mathrm{AF}$ & $1.41 \mathrm{E}-04$ & 35.30 & 10.8 & 21 -or & 1 \\
\hline $64 X-3,53-55$ & 555.75 & OT & $\mathrm{AF}$ & $2.93 \mathrm{E}-04$ & & & & 4 \\
\hline $66 X-1,70-72$ & 572.12 & OT & $\mathrm{AF}$ & $6.11 \mathrm{E}-04$ & & & & 4 \\
\hline $68 X-1,43-45$ & 581.55 & RM & $\mathrm{AF}$ & $4.70 \mathrm{E}-05$ & 34.08 & 16.4 & 21 -or & 2 \\
\hline $68 \times-2,37-39$ & 582.99 & OT & $\mathrm{AF}$ & $8.31 \mathrm{E}-05$ & & & & 4 \\
\hline $71 X-1,73-75$ & 610.65 & RM & $\mathrm{AF}$ & $7.69 \mathrm{E}-05$ & 14.54 & 20.4 & 35 -or & 3 \\
\hline $71 X-2,37-39$ & 611.79 & RM & $\mathrm{AF}$ & $1.39 \mathrm{E}-04$ & & & & 4 \\
\hline $71 X-3,77-79$ & 613.69 & RM & $\mathrm{AF}$ & $1.07 \mathrm{E}-04$ & & & & 4 \\
\hline $71 X-4,32-34$ & 614.34 & OT & $\mathrm{AF}$ & $3.73 \mathrm{E}-04$ & & & & 4 \\
\hline $72 X-1,103-105$ & 620.55 & RM & $\mathrm{AF}$ & $7.43 \mathrm{E}-05$ & 63.62 & 11.2 & 25 -or & 2 \\
\hline $72 X-2,103-105$ & 622.02 & RM & $\mathrm{AF}$ & $1.32 \mathrm{E}-04$ & 53.51 & 12.1 & 30 -or & 2 \\
\hline $72 X-3,100-102$ & 623.49 & RM & $\mathrm{AF}$ & $1.03 \mathrm{E}-04$ & 43.34 & 19.3 & 21 -or & 2 \\
\hline $72 X-4,41-43$ & 624.40 & OT & $\mathrm{AF}$ & $1.64 \mathrm{E}-04$ & & & & 4 \\
\hline $72 X-5,24-26$ & 625.73 & RM & $\mathrm{AF}$ & $1.72 \mathrm{E}-04$ & 55.48 & 8.0 & 21 -or & 2 \\
\hline $73 X-1,106-108$ & 630.18 & RM & $\mathrm{AF}$ & $3.40 \mathrm{E}-04$ & 56.76 & 8.4 & 30 -or & 1 \\
\hline $73 X-2,101-103$ & 631.63 & RM & $\mathrm{AF}$ & $5.26 \mathrm{E}-04$ & 21.55 & 5.9 & 21 -or & 1 \\
\hline $73 \times-3,43-45$ & 632.55 & RM & $\mathrm{AF}$ & $2.20 \mathrm{E}-04$ & 29.29 & 13.0 & 25 -or & 2 \\
\hline $73 X-5,101-103$ & 636.13 & OT & $\mathrm{AF}$ & $2.93 \mathrm{E}-03$ & & & & 4 \\
\hline $73 X-6,71-73$ & 637.33 & RM & $\mathrm{AF}$ & 7.27E-04 & 62.68 & 17.3 & 30 -or & 2 \\
\hline $75 X-1,48-50$ & 648.80 & RM & $\mathrm{AF}$ & $1.55 \mathrm{E}-03$ & 17.13 & 18.0 & $30-45$ & 3 \\
\hline $75 X-3,45-47$ & 651.77 & OT & $\mathrm{AF}$ & $1.75 \mathrm{E}-03$ & & & & 4 \\
\hline $76 X-1,48-50$ & 658.40 & RM & $\mathrm{AF}$ & $1.30 \mathrm{E}-03$ & 77.12 & 18.0 & 21 -or & 3 \\
\hline $76 X-2,48-50$ & 659.90 & RM & $\mathrm{AF}$ & $2.71 \mathrm{E}-03$ & 43.81 & 14.8 & 30 -or & 2 \\
\hline $76 X-7,48-50$ & 666.90 & RM & $\mathrm{AF}$ & $4.40 \mathrm{E}-03$ & 68.41 & 12.6 & 35 -or & 2 \\
\hline $77 X-1,90-92$ & 668.52 & RM & $\mathrm{AF}$ & $2.62 \mathrm{E}-04$ & 47.02 & 34.6 & 21-or & 3 \\
\hline $77 X-2,37-38$ & 669.48 & OT & $\mathrm{AF}$ & $7.08 \mathrm{E}-04$ & & & & 4 \\
\hline $78 X-1,70-72$ & 678.02 & RM & $\mathrm{AF}$ & $1.04 \mathrm{E}-04$ & 55.20 & 22.3 & 17 -or & 3 \\
\hline $78 X-2,42-44$ & 679.24 & OT & $\mathrm{AF}$ & $7.35 \mathrm{E}-04$ & & & & 4 \\
\hline $80 \times-1,57-59$ & 697.09 & OT & $\mathrm{AF}$ & $1.30 \mathrm{E}-03$ & & & & 4 \\
\hline $80 X-2,54-56$ & 698.56 & RM & $\mathrm{AF}$ & $2.73 \mathrm{E}-04$ & 34.89 & 7.7 & 21 -or & 2 \\
\hline $80 X-3,54-56$ & 700.06 & RM & $\mathrm{AF}$ & $1.78 \mathrm{E}-04$ & 70.71 & 9.9 & 30 -or & 2 \\
\hline $80 X-4,54-56$ & 701.56 & RM & $\mathrm{AF}$ & $1.40 \mathrm{E}-04$ & & & & 4 \\
\hline $80 X-5,54-56$ & 703.06 & RM & $\mathrm{AF}$ & $5.92 \mathrm{E}-04$ & 43.77 & 18.1 & 30 -or & 2 \\
\hline $81 X-1,44-46$ & 706.56 & RM & $\mathrm{AF}$ & $1.95 \mathrm{E}-04$ & 17.21 & 14.1 & 30 -or & 3 \\
\hline $81 X-2,49-51$ & 708.11 & OT & $\mathrm{AF}$ & $1.68 \mathrm{E}-03$ & 59.20 & 9.6 & 35 -or & 1 \\
\hline $81 X-3,44-46$ & 709.56 & RM & $\mathrm{AF}$ & $2.34 \mathrm{E}-04$ & 40.97 & 14.7 & 30 -or & 3 \\
\hline \multicolumn{9}{|l|}{ 317-U1352C- } \\
\hline $4 R-3,74-76$ & 597.66 & RM & $\mathrm{AF}$ & $7.62 \mathrm{E}-05$ & & & & 4 \\
\hline $8 R-1,33-35$ & 679.65 & RM & $\mathrm{TH}$ & & & & & 4 \\
\hline 10R-2, 112-114 & 700.48 & OT & $\mathrm{AF}$ & $4.83 \mathrm{E}-04$ & 25.30 & 10.7 & 40-or & 1 \\
\hline $12 \mathrm{R}-1,78-80$ & 718.90 & OT & $\mathrm{AF}$ & $1.14 \mathrm{E}-04$ & -14.60 & 6.9 & 150 -or & 1 \\
\hline $12 \mathrm{R}-1,81-83$ & 718.93 & RM & $\mathrm{AF}$ & $7.25 \mathrm{E}-06$ & & & & 4 \\
\hline 13R-1, 94-96 & 728.76 & OT & $\mathrm{AF}$ & $7.54 \mathrm{E}-05$ & & & & 4 \\
\hline 13R-1, 97-99 & 728.79 & RM & $\mathrm{AF}$ & $4.42 \mathrm{E}-05$ & -49.39 & 16.1 & 17-or & 2 \\
\hline $14 \mathrm{R}-2,65-67$ & 739.67 & OT & $\mathrm{AF}$ & $6.41 \mathrm{E}-05$ & & & & 4 \\
\hline $16 \mathrm{R}-2,46-48$ & 758.75 & RM & $\mathrm{TH}$ & & & & & 4 \\
\hline $16 \mathrm{R}-2,49-50$ & 758.77 & OT & $\mathrm{AF}$ & $1.19 \mathrm{E}-04$ & -7.20 & 8.3 & 15 -or & 3 \\
\hline $17 \mathrm{R}-2,24-26$ & 768.33 & RM & $\mathrm{AF}$ & $6.81 \mathrm{E}-05$ & & & & 4 \\
\hline $17 \mathrm{R}-2,28-30$ & 768.37 & OT & $\mathrm{AF}$ & $1.68 \mathrm{E}-04$ & -32.20 & 2.5 & 90 -or & 1 \\
\hline $18 \mathrm{R}-2,62-64$ & 778.44 & RM & $\mathrm{AF}$ & $9.45 \mathrm{E}-05$ & & & & 4 \\
\hline 19R-1, 75-77 & 786.77 & OT & $\mathrm{AF}$ & $1.18 \mathrm{E}-04$ & -65.20 & 16.0 & 20 -or & 3 \\
\hline $19 R-1,78-80$ & 786.80 & RM & $\mathrm{TH}$ & & & & & 4 \\
\hline
\end{tabular}


Table T2 (continued). (Continued on next page.)

\begin{tabular}{|c|c|c|c|c|c|c|c|c|}
\hline $\begin{array}{l}\text { Core, section, } \\
\text { interval }(\mathrm{cm})\end{array}$ & $\begin{array}{c}\text { Depth } \\
\text { CSF-A (m) }\end{array}$ & Lab & Treatment & $\begin{array}{c}\text { NRM } \\
\text { intensity } \\
(\mathrm{A} / \mathrm{m})\end{array}$ & $\begin{array}{c}\text { ChRM } \\
\text { inclination } \\
\left({ }^{\circ}\right)\end{array}$ & $\begin{array}{c}\text { MAD } \\
\left({ }^{\circ}\right)\end{array}$ & Steps & Class \\
\hline 20R-1, 73-75 & 796.45 & OT & $\mathrm{AF}$ & $1.81 \mathrm{E}-04$ & & & & 4 \\
\hline $20 \mathrm{R}-1,76-78$ & 796.48 & $\mathrm{RM}$ & $\mathrm{AF}$ & 5.97E-05 & -26.90 & 10.0 & 25 -or & 3 \\
\hline $21 \mathrm{R}-1,72-74$ & 806.14 & $\mathrm{RM}$ & $\mathrm{AF}$ & 5.87E-05 & -25.89 & 17.0 & 21 -or & 3 \\
\hline $21 \mathrm{R}-1,74-76$ & 806.16 & OT & $\mathrm{AF}$ & $6.63 \mathrm{E}-05$ & -19.10 & 9.4 & 90-or & 2 \\
\hline 22R-1, 49-51 & 815.51 & OT & $\mathrm{AF}$ & $4.28 \mathrm{E}-05$ & -43.30 & 28.0 & 50 -or & 3 \\
\hline $22 \mathrm{R}-1,51-53$ & 815.53 & $\mathrm{RM}$ & $\mathrm{AF}$ & $3.76 \mathrm{E}-05$ & -19.38 & 4.1 & 30-or & 3 \\
\hline 24R-2, 101-103 & 836.66 & $\mathrm{RM}$ & $\mathrm{AF}$ & $2.34 \mathrm{E}-05$ & -47.36 & 19.0 & 17-or & 3 \\
\hline 25R-1, 73-75 & 844.55 & $\mathrm{RM}$ & $\mathrm{TH}$ & & & & & 4 \\
\hline $26 \mathrm{R}-1,86-88$ & 854.28 & OT & $\mathrm{AF}$ & $1.42 \mathrm{E}-04$ & -6.00 & 4.3 & 300 -or & 1 \\
\hline $26 \mathrm{R}-1,88-90$ & 854.30 & $\mathrm{RM}$ & $\mathrm{TH}$ & & & & & 4 \\
\hline $27 \mathrm{R}-1,41-43$ & 863.43 & $\mathrm{RM}$ & $\mathrm{AF}$ & $4.14 \mathrm{E}-05$ & & & & 4 \\
\hline $28 \mathrm{R}-1,38-40$ & 873.00 & OT & $\mathrm{AF}$ & $3.65 \mathrm{E}-04$ & & & & 4 \\
\hline 30R-1, 71-73 & 892.53 & ОT & $\mathrm{AF}$ & $1.32 \mathrm{E}-04$ & & & & 4 \\
\hline $30 R-1,73-75$ & 892.55 & $\mathrm{RM}$ & $\mathrm{AF}$ & $3.11 \mathrm{E}-05$ & -78.36 & 3.3 & 25 -or & 3 \\
\hline $31 \mathrm{R}-2,27-29$ & 903.05 & $\mathrm{RM}$ & $\mathrm{AF}$ & 4.47E-04 & & & & 4 \\
\hline $31 \mathrm{R}-2,27-29$ & 903.05 & $\mathrm{RM}$ & $\mathrm{TH}$ & & & & & 4 \\
\hline $31 \mathrm{R}-2,29-31$ & 903.07 & OT & $\mathrm{AF}$ & 7.57E-05 & & & & 4 \\
\hline $32 \mathrm{R}-1,108-110$ & 912.00 & $\mathrm{RM}$ & $\mathrm{AF}$ & $1.45 \mathrm{E}-04$ & & & & 4 \\
\hline $32 \mathrm{R}-1,113-115$ & 912.05 & OT & $\mathrm{AF}$ & $6.40 \mathrm{E}-05$ & & & & 4 \\
\hline $33 R-2,104-106$ & 922.98 & $\mathrm{RM}$ & $\mathrm{AF}$ & $2.05 \mathrm{E}-04$ & 36.00 & 19.3 & 35 -or & 2 \\
\hline $34 \mathrm{R}-1,34-36$ & 930.46 & OT & $\mathrm{AF}$ & $1.92 \mathrm{E}-04$ & & & & 4 \\
\hline $34 R-1,40-42$ & 930.52 & $\mathrm{RM}$ & $\mathrm{AF}$ & $7.15 \mathrm{E}-05$ & & & & 4 \\
\hline $35 R-3,53-55$ & 943.25 & $\mathrm{RM}$ & $\mathrm{TH}$ & & & & & 4 \\
\hline $35 R-3,56-57$ & 943.27 & OT & $\mathrm{AF}$ & $9.52 \mathrm{E}-05$ & 31.80 & 15.5 & 180 -or & 2 \\
\hline $36 \mathrm{R}-2,52-54$ & 951.44 & $\mathrm{RM}$ & $\mathrm{AF}$ & $2.78 \mathrm{E}-05$ & -23.44 & 6.5 & 17-or & 3 \\
\hline $37 R-4,53-55$ & 963.41 & $\mathrm{RM}$ & $\mathrm{AF}$ & $2.24 \mathrm{E}-04$ & & & & 4 \\
\hline $37 R-4,55-57$ & 963.43 & OT & $\mathrm{AF}$ & $5.50 \mathrm{E}-04$ & 29.50 & 28.8 & 40 -or & 3 \\
\hline $38 \mathrm{R}-1,122-124$ & 969.74 & $\mathrm{RM}$ & $\mathrm{AF}$ & $1.04 \mathrm{E}-04$ & -26.42 & 13.9 & 35 -or & 3 \\
\hline $38 \mathrm{R}-3,35-37$ & 971.87 & $\mathrm{RM}$ & $\mathrm{AF}$ & $5.86 \mathrm{E}-05$ & & & & 4 \\
\hline $38 R-5,76-78$ & 975.28 & OT & $\mathrm{AF}$ & $5.52 \mathrm{E}-05$ & & & & 4 \\
\hline $38 R-5,78-80$ & 975.30 & $\mathrm{RM}$ & $\mathrm{AF}$ & $3.48 \mathrm{E}-05$ & & & & 4 \\
\hline 38R-7, 10-12 & 977.62 & $\mathrm{RM}$ & $\mathrm{TH}$ & & & & & 4 \\
\hline $39 \mathrm{R}-1,112-114$ & 979.24 & $\mathrm{RM}$ & $\mathrm{AF}$ & 4.89E-05 & & & & 4 \\
\hline $39 R-3,31-32$ & 981.42 & OT & $\mathrm{AF}$ & $1.26 \mathrm{E}-04$ & 62.30 & 14.9 & 120 -or & 3 \\
\hline $39 R-3,33-35$ & 981.45 & $\mathrm{RM}$ & $\mathrm{AF}$ & $7.64 \mathrm{E}-05$ & 31.54 & 18.0 & 40-or & 2 \\
\hline $39 R-5,34-36$ & 984.46 & $\mathrm{RM}$ & $\mathrm{TH}$ & & & & & 4 \\
\hline 39R-7, 5-7 & 987.17 & OT & $\mathrm{AF}$ & $1.44 \mathrm{E}-04$ & & & & 4 \\
\hline $40 R-1,83-85$ & 988.55 & $\mathrm{RM}$ & $\mathrm{TH}$ & & & & & 4 \\
\hline $40 R-3,54-56$ & 991.07 & OT & $\mathrm{AF}$ & $1.82 \mathrm{E}-04$ & & & & 4 \\
\hline $40 R-3,56-58$ & 991.09 & $\mathrm{RM}$ & $\mathrm{AF}$ & $7.91 \mathrm{E}-05$ & -29.09 & 18.9 & 45 -or & 3 \\
\hline $41 \mathrm{R}-1,98-100$ & 998.30 & OT & $\mathrm{AF}$ & $1.29 \mathrm{E}-04$ & 45.20 & 11.8 & 240 -or & 2 \\
\hline $41 \mathrm{R}-1,100-102$ & 998.32 & $\mathrm{RM}$ & $\mathrm{AF}$ & $3.99 \mathrm{E}-05$ & & & & 4 \\
\hline $41 R-3,40-42$ & 1000.72 & $\mathrm{RM}$ & $\mathrm{TH}$ & & & & & 4 \\
\hline $41 \mathrm{R}-4,102-104$ & 1002.84 & $\mathrm{RM}$ & $\mathrm{AF}$ & $1.30 \mathrm{E}-04$ & 20.68 & 13.9 & 45 -or & 3 \\
\hline $41 \mathrm{R}-4,102-104$ & 1002.84 & $\mathrm{RM}$ & $\mathrm{AF}$ & 7.17E-05 & 20.68 & 13.9 & 45 -or & 3 \\
\hline 42R-3, 110-112 & 1010.56 & $\mathrm{RM}$ & $\mathrm{AF}$ & $7.71 \mathrm{E}-05$ & & & & 4 \\
\hline 42R-3, 114-116 & 1010.60 & OT & $\mathrm{AF}$ & $1.40 \mathrm{E}-04$ & 59.70 & 11.7 & 20-or & 3 \\
\hline $43 R-3,45-46$ & 1019.39 & OT & $\mathrm{TH}$ & $1.59 \mathrm{E}-04$ & -79.40 & 7.3 & 300 -or & 2 \\
\hline $43 R-3,48-49$ & 1019.42 & $\mathrm{RM}$ & $\mathrm{TH}$ & & & & & 4 \\
\hline $44 R-1,44-46$ & 1026.56 & $\mathrm{RM}$ & $\mathrm{AF}$ & $7.60 \mathrm{E}-05$ & 21.59 & 11.7 & 40 -or & 3 \\
\hline 44R-6, 29-31 & 1033.65 & $\mathrm{RM}$ & $\mathrm{AF}$ & $9.86 \mathrm{E}-05$ & & & & 4 \\
\hline $45 R-3,56-58$ & 1039.15 & $\mathrm{RM}$ & $\mathrm{AF}$ & $1.43 \mathrm{E}-05$ & & & & 4 \\
\hline $45 R-3,61-63$ & 1039.20 & OT & $\mathrm{AF}$ & $1.18 \mathrm{E}-04$ & & & & 4 \\
\hline $46 \mathrm{R}-3,79-81$ & 1049.05 & OT & $\mathrm{TH}$ & $7.71 \mathrm{E}-05$ & & & & 4 \\
\hline $46 \mathrm{R}-3,82-84$ & 1049.08 & $\mathrm{RM}$ & $\mathrm{AF}$ & $5.13 \mathrm{E}-05$ & & & & 4 \\
\hline $47 R-3,40-42$ & 1057.94 & $\mathrm{RM}$ & $\mathrm{AF}$ & $9.43 \mathrm{E}-05$ & & & & 4 \\
\hline $47 R-3,42-44$ & 1057.96 & OT & $\mathrm{AF}$ & $2.03 \mathrm{E}-04$ & 59.20 & 21.9 & 70-or & 3 \\
\hline $48 \mathrm{R}-2,76-78$ & 1066.66 & OT & $\mathrm{TH}$ & $6.44 \mathrm{E}-05$ & 27.60 & 9.7 & 240 -or & 3 \\
\hline $48 \mathrm{R}-2,78-80$ & 1066.68 & $\mathrm{RM}$ & $\mathrm{TH}$ & & & & & 4 \\
\hline $48 R-4,48-50$ & 1068.86 & $\mathrm{RM}$ & $\mathrm{AF}$ & $7.69 \mathrm{E}-05$ & & & & 4 \\
\hline 49R-1, 76-78 & 1074.58 & $\mathrm{RM}$ & $\mathrm{AF}$ & $5.78 \mathrm{E}-05$ & -36.13 & 17.6 & 17-or & 2 \\
\hline $49 R-2,102-104$ & 1076.31 & OT & $\mathrm{AF}$ & $1.58 \mathrm{E}-04$ & & & & 4 \\
\hline 49R-2, 105-107 & 1076.34 & $\mathrm{RM}$ & $\mathrm{AF}$ & $1.30 \mathrm{E}-04$ & & & & 4 \\
\hline $50 R-2,87-89$ & 1085.69 & $\mathrm{RM}$ & $\mathrm{AF}$ & $1.70 \mathrm{E}-04$ & & & & 4 \\
\hline $50 \mathrm{R}-4,124-126$ & 1089.06 & $\mathrm{RM}$ & $\mathrm{AF}$ & $1.95 \mathrm{E}-04$ & 15.34 & 14.3 & 40 -or & 3 \\
\hline 50R-6, 130-132 & 1092.12 & $\mathrm{RM}$ & $\mathrm{TH}$ & & & & & 4 \\
\hline $51 \mathrm{R}-2,51-53$ & 1094.93 & OT & $\mathrm{TH}$ & $1.87 \mathrm{E}-04$ & 29.90 & 19.2 & 270 -or & 2 \\
\hline $51 R-2,53-55$ & 1094.95 & $\mathrm{RM}$ & $\mathrm{TH}$ & & & & & 4 \\
\hline
\end{tabular}


Table T2 (continued). (Continued on next page.)

\begin{tabular}{|c|c|c|c|c|c|c|c|c|}
\hline $\begin{array}{l}\text { Core, section, } \\
\text { interval }(\mathrm{cm})\end{array}$ & $\begin{array}{l}\text { Depth } \\
\text { CSF-A (m) }\end{array}$ & Lab & Treatment & $\begin{array}{c}\text { NRM } \\
\text { intensity } \\
(\mathrm{A} / \mathrm{m})\end{array}$ & $\begin{array}{c}\text { ChRM } \\
\text { inclination } \\
\left(^{\circ}\right)\end{array}$ & $\begin{array}{c}\text { MAD } \\
\left({ }^{\circ}\right)\end{array}$ & Steps & Class \\
\hline 51R-4, 59-61 & 1097.98 & RM & $\mathrm{AF}$ & $5.88 \mathrm{E}-05$ & & & & 4 \\
\hline $52 \mathrm{R}-2,38-40$ & 1104.30 & OT & $\mathrm{AF}$ & $9.16 \mathrm{E}-05$ & & & & 4 \\
\hline $52 \mathrm{R}-2,40-42$ & 1104.32 & $\mathrm{RM}$ & $\mathrm{AF}$ & $9.31 \mathrm{E}-06$ & -34.29 & 22.2 & 25 -or & 2 \\
\hline $53 \mathrm{R}-1,38-40$ & 1112.50 & OT & TH & $7.82 \mathrm{E}-05$ & 43.00 & 11.9 & 300 -or & 3 \\
\hline $53 \mathrm{R}-1,40-42$ & 1112.52 & $\mathrm{RM}$ & $\mathrm{AF}$ & $6.67 \mathrm{E}-05$ & & & & 4 \\
\hline 54R-2, 17-19 & 1123.39 & OT & $\mathrm{AF}$ & $1.44 \mathrm{E}-04$ & & & & 4 \\
\hline 54R-2, 19-21 & 1123.41 & $\mathrm{RM}$ & $\mathrm{AF}$ & $5.77 \mathrm{E}-05$ & -73.05 & 10.0 & 17-or & 2 \\
\hline $54 \mathrm{R}-4,54-56$ & 1126.74 & $\mathrm{RM}$ & TH & & & & & 4 \\
\hline $56 \mathrm{R}-5,52-54$ & 1147.32 & OT & TH & $2.79 \mathrm{E}-04$ & 59.00 & 15.9 & 330 -or & 3 \\
\hline $56 \mathrm{R}-5,54-56$ & 1147.34 & $\mathrm{RM}$ & $\mathrm{AF}$ & $1.76 \mathrm{E}-04$ & 59.26 & 7.0 & 25 -or & 2 \\
\hline 57R-4, 45-47 & 1154.79 & $\mathrm{RM}$ & $\mathrm{AF}$ & $3.12 \mathrm{E}-05$ & & & & 4 \\
\hline $57 \mathrm{R}-4,45-47$ & 1154.79 & $\mathrm{RM}$ & TH & & & & & 4 \\
\hline $57 \mathrm{R}-4,48-50$ & 1154.82 & OT & $\mathrm{AF}$ & $1.01 \mathrm{E}-04$ & & & & 4 \\
\hline $58 \mathrm{R}-4,78-80$ & 1164.07 & OT & TH & $1.72 \mathrm{E}-04$ & & & & 4 \\
\hline 59R-5, 101-103 & 1176.73 & $\mathrm{RM}$ & $\mathrm{AF}$ & $8.03 \mathrm{E}-05$ & -37.53 & 14.2 & 35 -or & 1 \\
\hline 59R-5, 103-105 & 1176.75 & OT & $\mathrm{AF}$ & $6.15 \mathrm{E}-05$ & & & & 4 \\
\hline $60 \mathrm{R}-4,48-50$ & 1184.40 & OT & $\mathrm{AF}$ & $1.07 \mathrm{E}-04$ & -34.80 & 11.8 & 70 -or & 2 \\
\hline $60 \mathrm{R}-4,51-53$ & 1184.43 & $\mathrm{RM}$ & TH & & & & & 4 \\
\hline $61 \mathrm{R}-3,50-52$ & 1192.52 & OT & $\mathrm{AF}$ & $1.43 \mathrm{E}-04$ & & & & 4 \\
\hline $61 \mathrm{R}-3,52-54$ & 1192.54 & RM & $\mathrm{AF}$ & $6.53 \mathrm{E}-06$ & & & & 4 \\
\hline 61R-6, 89-91 & 1197.41 & $\mathrm{RM}$ & TH & & & & & 4 \\
\hline $62 \mathrm{R}-2,14-16$ & 1200.26 & $\mathrm{RM}$ & $\mathrm{AF}$ & 5.77E-05 & -16.27 & 13.2 & 40 -or & 3 \\
\hline $62 \mathrm{R}-4,30-32$ & 1203.42 & OT & $\mathrm{AF}$ & 1.07E-04 & 33.60 & 12.3 & 270 -or & 2 \\
\hline $62 \mathrm{R}-4,32-34$ & 1203.44 & $\mathrm{RM}$ & TH & & & & & 4 \\
\hline 70R-3, 87-89 & 1250.59 & $\mathrm{RM}$ & $\mathrm{AF}$ & $8.72 \mathrm{E}-05$ & 68.25 & 14.1 & 30 -or & 2 \\
\hline 70R-6, 28-30 & 1254.46 & $\mathrm{RM}$ & $\mathrm{AF}$ & $3.36 \mathrm{E}-05$ & & & & 4 \\
\hline $71 \mathrm{R}-6,31-33$ & 1264.13 & $\mathrm{RM}$ & TH & & & & & 4 \\
\hline $71 \mathrm{R}-6,33-35$ & 1264.15 & От & TH & $2.00 \mathrm{E}-04$ & & & & 4 \\
\hline 73R-1, 141-143 & 1276.43 & $\mathrm{RM}$ & $\mathrm{AF}$ & $2.17 \mathrm{E}-04$ & 42.84 & 20.1 & 25 -or & 2 \\
\hline $73 R-4,71-73$ & 1280.23 & RM & $\mathrm{AF}$ & $5.03 \mathrm{E}-05$ & & & & 4 \\
\hline 76R-1, 24-26 & 1304.16 & $\mathrm{RM}$ & TH & & & & & 4 \\
\hline 76R-1, 26-28 & 1304.18 & OT & $\mathrm{AF}$ & $9.56 \mathrm{E}-04$ & 49.80 & 9.6 & 35 -or & 2 \\
\hline 77R-1, 127-129 & 1309.69 & OT & $\mathrm{AF}$ & $3.26 \mathrm{E}-04$ & 65.50 & 19.0 & 45 -or & 3 \\
\hline 77R-1, 137-139 & 1309.79 & $\mathrm{RM}$ & $\mathrm{AF}$ & $1.84 \mathrm{E}-04$ & 27.92 & 7.0 & 30 -or & 2 \\
\hline $78 R-3,61-64$ & 1316.98 & OT & TH & $4.15 \mathrm{E}-04$ & 80.50 & 2.0 & 180 -or & 2 \\
\hline 78R-3, 102-104 & 1317.38 & $\mathrm{RM}$ & $\mathrm{AF}$ & $9.86 \mathrm{E}-06$ & & & & 4 \\
\hline 79R-1, 32-34 & 1318.97 & $\mathrm{RM}$ & TH & & & & & 4 \\
\hline $80 \mathrm{R}-1,138-140$ & 1324.30 & $\mathrm{RM}$ & $\mathrm{AF}$ & $2.16 \mathrm{E}-04$ & 62.22 & 12.5 & 21 -or & 2 \\
\hline 80R-1, 140-142 & 1324.32 & OT & $\mathrm{AF}$ & $3.30 \mathrm{E}-04$ & 71.60 & 1.6 & 35 -or & 2 \\
\hline $81 \mathrm{R}-1,110-112$ & 1328.62 & $\mathrm{RM}$ & TH & & & & & 4 \\
\hline 85R-2, 59-61 & 1348.81 & OT & $\mathrm{AF}$ & $2.48 \mathrm{E}-04$ & & & & 4 \\
\hline 85R-2, 62-64 & 1348.84 & $\mathrm{RM}$ & $\mathrm{AF}$ & $1.65 \mathrm{E}-04$ & 64.03 & 8.3 & 21 -or & 2 \\
\hline $85 R-4,63-65$ & 1351.85 & $\mathrm{RM}$ & TH & & & & & 4 \\
\hline 86R-2, 103-105 & 1354.21 & OT & $\mathrm{AF}$ & $3.15 \mathrm{E}-04$ & & & & 4 \\
\hline 86R-2, 105-107 & 1354.23 & RM & $\mathrm{AF}$ & $2.24 \mathrm{E}-04$ & -21.53 & 16.6 & 40 -or & 3 \\
\hline 87R-3, 49-51 & 1364.91 & $\mathrm{RM}$ & TH & & & & & 4 \\
\hline 87R-5, 100-102 & 1368.42 & OT & TH & $3.16 \mathrm{E}-04$ & & & & 4 \\
\hline 87R-5, 102-104 & 1368.44 & $\mathrm{RM}$ & TH & & & & & 4 \\
\hline $88 \mathrm{R}-4,85-87$ & 1376.37 & OT & $\mathrm{AF}$ & $2.77 \mathrm{E}-04$ & & & & 4 \\
\hline 88R-4, 87-89 & 1376.39 & $\mathrm{RM}$ & $\mathrm{AF}$ & $1.80 \mathrm{E}-04$ & 33.33 & 5.5 & 17-or & 3 \\
\hline $88 \mathrm{R}-6,49-51$ & 1379.01 & $\mathrm{RM}$ & $\mathrm{AF}$ & $1.96 \mathrm{E}-05$ & & & & 4 \\
\hline 89R-3, 101-103 & 1384.45 & RM & $\mathrm{AF}$ & $1.28 \mathrm{E}-04$ & & & & 4 \\
\hline 89R-3, 105-107 & 1384.49 & OT & $\mathrm{AF}$ & $1.60 \mathrm{E}-04$ & & & & 4 \\
\hline 90R-1, 95-97 & 1391.17 & $\mathrm{RM}$ & $\mathrm{AF}$ & $7.78 \mathrm{E}-05$ & & & & 4 \\
\hline 90R-1, 98-100 & 1391.20 & OT & $\mathrm{AF}$ & $3.11 \mathrm{E}-04$ & & & & 4 \\
\hline 91R-3, 89-91 & 1403.61 & $\mathrm{RM}$ & TH & & & & & 4 \\
\hline $94 \mathrm{R}-4,54-56$ & 1433.66 & $\mathrm{RM}$ & TH & & & & & 4 \\
\hline $94 R-4,57-59$ & 1433.69 & OT & TH & $2.35 \mathrm{E}-04$ & & & & 4 \\
\hline $95 \mathrm{R}-2,61-63$ & 1440.43 & $\mathrm{RM}$ & $\mathrm{AF}$ & $2.65 \mathrm{E}-04$ & 15.54 & 9.7 & 40 -or & 3 \\
\hline $95 R-4,112-114$ & 1443.94 & OT & $\mathrm{AF}$ & 1.47E-04 & & & & 4 \\
\hline $99 \mathrm{R}-1,71-73$ & 1477.73 & $\mathrm{RM}$ & TH & & & & & 4 \\
\hline 103R-5, 108-110 & 1513.20 & OT & $\mathrm{AF}$ & $7.29 \mathrm{E}-04$ & & & & 4 \\
\hline 103R-5, 111-113 & 1513.23 & $\mathrm{RM}$ & $\mathrm{AF}$ & $6.25 \mathrm{E}-04$ & 76.27 & 19.4 & 30 -or & 2 \\
\hline 104R-2, 84-86 & 1518.16 & $\mathrm{RM}$ & $\mathrm{AF}$ & $5.86 \mathrm{E}-05$ & & & & 4 \\
\hline 104R-2, 127-129 & 1518.59 & OT & $\mathrm{AF}$ & $5.20 \mathrm{E}-04$ & & & & 4 \\
\hline 104R-5, 89-91 & 1522.71 & $\mathrm{RM}$ & TH & & & & & 4 \\
\hline 105R-2, 35-37 & 1527.37 & OT & $\mathrm{AF}$ & $8.11 \mathrm{E}-04$ & & & & 4 \\
\hline $105 \mathrm{R}-2,38-40$ & 1527.40 & $\mathrm{RM}$ & $\mathrm{AF}$ & $8.43 \mathrm{E}-05$ & 54.71 & 9.8 & 25 -or & 2 \\
\hline
\end{tabular}


Table T2 (continued). (Continued on next page.)

\begin{tabular}{|c|c|c|c|c|c|c|c|c|}
\hline $\begin{array}{l}\text { Core, section, } \\
\text { interval }(\mathrm{cm})\end{array}$ & $\begin{array}{c}\text { Depth } \\
\text { CSF-A (m) }\end{array}$ & Lab & Treatment & $\begin{array}{c}\text { NRM } \\
\text { intensity } \\
(\mathrm{A} / \mathrm{m})\end{array}$ & $\begin{array}{c}\text { ChRM } \\
\text { inclination } \\
\left({ }^{\circ}\right)\end{array}$ & $\begin{array}{c}\text { MAD } \\
\left({ }^{\circ}\right)\end{array}$ & Steps & Class \\
\hline $105 R-5,27-30$ & 1531.80 & $\mathrm{RM}$ & $\mathrm{AF}$ & 4.47E-04 & 74.72 & 8.6 & 21 -or & 1 \\
\hline $106 R-5,24-26$ & 1541.32 & $\mathrm{RM}$ & $\mathrm{AF}$ & $1.09 \mathrm{E}-04$ & & & & 4 \\
\hline 106R-5, 27-29 & 1541.35 & OT & $\mathrm{AF}$ & $6.06 \mathrm{E}-04$ & & & & 4 \\
\hline 107R-3, 134-136 & 1549.26 & $\mathrm{RM}$ & $\mathrm{TH}$ & & & & & 4 \\
\hline 107R-3, 137-139 & 1549.29 & OT & $\mathrm{TH}$ & $1.40 \mathrm{E}-03$ & & & & 4 \\
\hline $107 R-5,79-80$ & 1551.70 & OT & $\mathrm{AF}$ & $5.11 \mathrm{E}-04$ & & & & 4 \\
\hline $107 R-5,81-83$ & 1551.73 & $\mathrm{RM}$ & $\mathrm{AF}$ & $3.73 \mathrm{E}-04$ & & & & 4 \\
\hline 108R-2, 97-99 & 1557.04 & $\mathrm{RM}$ & $\mathrm{TH}$ & & & & & 4 \\
\hline $109 R-3,06-08$ & 1567.30 & OT & $\mathrm{TH}$ & $6.91 \mathrm{E}-04$ & & & & 4 \\
\hline 109R-3, 10-12 & 1567.31 & $\mathrm{RM}$ & $\mathrm{AF}$ & $3.67 \mathrm{E}-04$ & 68.62 & 10.2 & 21 -or & 2 \\
\hline $110 \mathrm{R}-2,21-23$ & 1575.80 & $\mathrm{RM}$ & $\mathrm{TH}$ & & & & & 4 \\
\hline $110 \mathrm{R}-2,24-26$ & 1575.83 & OT & $\mathrm{AF}$ & $5.74 \mathrm{E}-04$ & & & & 4 \\
\hline $111 R-2,10-12$ & 1585.36 & $\mathrm{RM}$ & $\mathrm{AF}$ & $3.35 \mathrm{E}-04$ & 70.50 & 23.6 & 25 -or & 2 \\
\hline $111 R-2,13-15$ & 1585.39 & OT & $\mathrm{TH}$ & $5.52 \mathrm{E}-04$ & & & & 4 \\
\hline $112 \mathrm{R}-1,124-126$ & 1594.76 & $\mathrm{RM}$ & $\mathrm{AF}$ & $2.52 \mathrm{E}-04$ & 74.23 & 7.2 & 21 -or & 2 \\
\hline $112 R-3,21-23$ & 1596.65 & OT & $\mathrm{AF}$ & $8.50 \mathrm{E}-04$ & & & & 4 \\
\hline 113R-2, 95-97 & 1605.67 & OT & $\mathrm{TH}$ & $1.10 \mathrm{E}-03$ & & & & 4 \\
\hline $113 R-6,63-65$ & 1611.20 & $\mathrm{RM}$ & $\mathrm{TH}$ & & & & & 4 \\
\hline $114 \mathrm{R}-1,116-118$ & 1614.08 & OT & $\mathrm{AF}$ & $4.41 \mathrm{E}-04$ & & & & 4 \\
\hline $114 R-4,7-9$ & 1617.49 & $\mathrm{RM}$ & $\mathrm{AF}$ & $7.28 \mathrm{E}-04$ & 75.02 & 9.6 & 25 -or & 1 \\
\hline 115R-4, 97-99 & 1628.19 & $\mathrm{RM}$ & $\mathrm{TH}$ & & & & & 4 \\
\hline $116 R-3,65-67$ & 1636.07 & OT & $\mathrm{TH}$ & $1.77 \mathrm{E}-03$ & & & & 4 \\
\hline $116 \mathrm{R}-3,67-69$ & 1636.09 & $\mathrm{RM}$ & $\mathrm{AF}$ & $2.75 \mathrm{E}-04$ & 62.74 & 3.7 & 60 -or & 2 \\
\hline $117 R-3,46-48$ & 1644.75 & OT & $\mathrm{AF}$ & $1.86 \mathrm{E}-03$ & & & & 4 \\
\hline $117 R-3,48-50$ & 1644.77 & $\mathrm{RM}$ & $\mathrm{AF}$ & $1.13 \mathrm{E}-03$ & 70.58 & 5.4 & 30 -or & 1 \\
\hline $118 \mathrm{R}-7,18-20$ & 1659.72 & OT & $\mathrm{TH}$ & $5.70 \mathrm{E}-04$ & & & & 4 \\
\hline 118R-7, 21-23 & 1659.73 & $\mathrm{RM}$ & $\mathrm{AF}$ & $4.68 \mathrm{E}-04$ & 80.20 & 26.0 & 20-or & 2 \\
\hline 119R-2, 140-142 & 1664.42 & $\mathrm{RM}$ & $\mathrm{TH}$ & & & & & 4 \\
\hline 119R-6, 110-112 & 1670.12 & $\mathrm{RM}$ & $\mathrm{AF}$ & $1.27 \mathrm{E}-03$ & 77.54 & 7.2 & 40 -or & 1 \\
\hline $122 \mathrm{R}-1,75-77$ & 1688.87 & $\mathrm{RM}$ & $\mathrm{TH}$ & & & & & 4 \\
\hline 122R-1, 77-79 & 1688.89 & OT & $\mathrm{AF}$ & $2.55 \mathrm{E}-03$ & & & & 4 \\
\hline $123 R-2,111-113$ & 1695.13 & $\mathrm{RM}$ & $\mathrm{AF}$ & $1.82 \mathrm{E}-03$ & 71.29 & 3.9 & 30 -or & 1 \\
\hline $123 R-2,123-125$ & 1695.25 & OT & $\mathrm{AF}$ & $2.88 \mathrm{E}-03$ & & & & 4 \\
\hline $124 \mathrm{R}-2,81-83$ & 1699.77 & $\mathrm{RM}$ & $\mathrm{AF}$ & $6.39 \mathrm{E}-04$ & 70.99 & 7.6 & 21 -or & 1 \\
\hline $124 \mathrm{R}-2,100-102$ & 1699.96 & OT & $\mathrm{TH}$ & $6.13 \mathrm{E}-03$ & & & & 4 \\
\hline $125 R-5,50-52$ & 1713.52 & OT & $\mathrm{AF}$ & $2.83 \mathrm{E}-03$ & & & & 4 \\
\hline $125 R-5,57-59$ & 1713.59 & $\mathrm{RM}$ & $\mathrm{AF}$ & $1.62 \mathrm{E}-03$ & 84.37 & 4.5 & 35 -or & 1 \\
\hline 126R-2, 129-131 & 1719.41 & $\mathrm{RM}$ & $\mathrm{AF}$ & $1.27 \mathrm{E}-03$ & 85.69 & 5.5 & 25 -or & 1 \\
\hline 126R-6, 67-69 & 1724.46 & $\mathrm{RM}$ & $\mathrm{AF}$ & $6.26 \mathrm{E}-04$ & 83.09 & 8.2 & 21 -or & 1 \\
\hline 126R-6, 79-81 & 1724.58 & OT & $\mathrm{TH}$ & $7.32 \mathrm{E}-04$ & & & & 4 \\
\hline $127 \mathrm{R}-1,26-28$ & 1726.48 & OT & $\mathrm{AF}$ & $8.21 \mathrm{E}-04$ & & & & 4 \\
\hline $127 \mathrm{R}-1,28-30$ & 1726.50 & $\mathrm{RM}$ & $\mathrm{TH}$ & & & & & 4 \\
\hline $128 \mathrm{R}-2,114-116$ & 1738.46 & $\mathrm{RM}$ & $\mathrm{AF}$ & $6.12 \mathrm{E}-04$ & 82.37 & 3.0 & 30 -or & 1 \\
\hline $128 \mathrm{R}-3,9-11$ & 1738.91 & $\mathrm{RM}$ & $\mathrm{AF}$ & $6.80 \mathrm{E}-04$ & 87.14 & 6.8 & 25 -or & 1 \\
\hline $128 R-4,45-47$ & 1740.63 & OT & $\mathrm{AF}$ & $1.86 \mathrm{E}-03$ & & & & 4 \\
\hline $129 \mathrm{R}-1,43-45$ & 1745.85 & $\mathrm{RM}$ & $\mathrm{AF}$ & $6.42 \mathrm{E}-04$ & 83.94 & 5.0 & 21 -or & 1 \\
\hline $129 \mathrm{R}-2,42-44$ & 1747.28 & OT & $\mathrm{TH}$ & $1.37 \mathrm{E}-03$ & & & & 4 \\
\hline 130R-3, 110-112 & 1759.10 & $\mathrm{RM}$ & $\mathrm{AF}$ & $3.49 \mathrm{E}-04$ & 62.68 & 29.4 & $21-50$ & 2 \\
\hline 130R-3, 114-116 & 1759.14 & $\mathrm{RM}$ & $\mathrm{AF}$ & $7.60 \mathrm{E}-04$ & 50.14 & 8.6 & 25 -or & 2 \\
\hline 131R-2, 75-77 & 1766.79 & $\mathrm{RM}$ & $\mathrm{AF}$ & $1.98 \mathrm{E}-04$ & 82.60 & 25.1 & 25 -or & 2 \\
\hline $131 R-2,84-86$ & 1766.86 & OT & $\mathrm{AF}$ & $3.90 \mathrm{E}-04$ & & & & 4 \\
\hline 132R-3, 17-19 & 1776.96 & $\mathrm{RM}$ & $\mathrm{TH}$ & & & & & 4 \\
\hline 132R-3, 19-21 & 1776.98 & OT & $\mathrm{AF}$ & $4.08 \mathrm{E}-04$ & & & & 4 \\
\hline 133R-4, 129-131 & 1789.39 & OT & $\mathrm{AF}$ & $3.03 \mathrm{E}-04$ & & & & 4 \\
\hline $133 R-4,125-127$ & 1789.43 & $\mathrm{RM}$ & $\mathrm{AF}$ & $2.08 \mathrm{E}-04$ & 84.77 & 19.4 & 30 -or & 2 \\
\hline 134R-3, 10-11 & 1795.99 & $\mathrm{RM}$ & $\mathrm{AF}$ & $2.34 \mathrm{E}-04$ & 59.74 & 5.5 & 30 -or & 2 \\
\hline $134 \mathrm{R}-3,12-14$ & 1796.02 & OT & $\mathrm{AF}$ & $2.52 \mathrm{E}-04$ & & & & 4 \\
\hline 135R-5, 18-20 & 1808.94 & OT & $\mathrm{TH}$ & $2.88 \mathrm{E}-04$ & & & & 4 \\
\hline 135R-5, 21-23 & 1808.97 & $\mathrm{RM}$ & $\mathrm{AF}$ & $2.44 \mathrm{E}-04$ & 80.41 & 15.2 & 30 -or & 2 \\
\hline 136R-2, 90-92 & 1815.04 & $\mathrm{RM}$ & $\mathrm{AF}$ & $6.78 \mathrm{E}-05$ & & & & 4 \\
\hline 136R-3, 107-109 & 1816.68 & OT & $\mathrm{AF}$ & 4.77E-04 & & & & 4 \\
\hline 137R-1, 112-114 & 1823.44 & $\mathrm{RM}$ & $\mathrm{AF}$ & $2.24 \mathrm{E}-04$ & 71.78 & 10.0 & 30 -or & 2 \\
\hline 137R-5, 117-119 & 1829.38 & OT & $\mathrm{TH}$ & $3.94 \mathrm{E}-04$ & & & & 4 \\
\hline $138 \mathrm{R}-1,30-32$ & 1832.22 & $\mathrm{RM}$ & $\mathrm{AF}$ & $5.38 \mathrm{E}-04$ & 84.20 & 2.6 & 25 -or & 1 \\
\hline 138R-4, 77-79 & 1837.18 & $\mathrm{RM}$ & $\mathrm{TH}$ & & & & & 4 \\
\hline 138R-7, 24-26 & 1841.07 & $\mathrm{RM}$ & $\mathrm{AF}$ & $8.83 \mathrm{E}-05$ & & & & 4 \\
\hline 139R-1, 116-118 & 1842.68 & OT & $\mathrm{AF}$ & $1.60 \mathrm{E}-04$ & 24.70 & 8.8 & 30 -or & 2 \\
\hline 139R-1, 125-127 & 1842.77 & OT & $\mathrm{AF}$ & $2.32 \mathrm{E}-04$ & 48.00 & 14.2 & 50 -or & 2 \\
\hline
\end{tabular}


Table T2 (continued).

\begin{tabular}{|c|c|c|c|c|c|c|c|c|}
\hline $\begin{array}{l}\text { Core, section, } \\
\text { interval }(\mathrm{cm})\end{array}$ & $\begin{array}{c}\text { Depth } \\
\text { CSF-A (m) }\end{array}$ & Lab & Treatment & $\begin{array}{c}\text { NRM } \\
\text { intensity } \\
(\mathrm{A} / \mathrm{m})\end{array}$ & $\begin{array}{c}\text { ChRM } \\
\text { inclination } \\
\left(^{\circ}\right)\end{array}$ & $\begin{array}{c}\text { MAD } \\
\left({ }^{\circ}\right)\end{array}$ & Steps & Class \\
\hline $139 R-3,83-85$ & 1845.31 & ОТ & $\mathrm{TH}$ & $1.57 \mathrm{E}-04$ & 45.00 & 13.0 & 240-or & 2 \\
\hline $139 R-5,49-51$ & 1847.87 & OT & $\mathrm{AF}$ & 2.27E-04 & & & & 4 \\
\hline 139R-5, 72-74 & 1848.10 & $\mathrm{RM}$ & $\mathrm{AF}$ & $1.35 \mathrm{E}-04$ & -52.93 & 14.3 & 17-or & 2 \\
\hline 140R-1, 18-20 & 1851.30 & $\mathrm{RM}$ & $\mathrm{AF}$ & $5.02 \mathrm{E}-05$ & & & & 4 \\
\hline 140R-1, 22-24 & 1851.34 & OT & $\mathrm{AF}$ & $1.17 \mathrm{E}-04$ & & & & 4 \\
\hline $141 R-2,35-37$ & 1862.39 & $\mathrm{RM}$ & $\mathrm{AF}$ & $4.40 \mathrm{E}-06$ & & & & 4 \\
\hline 143R-2, 39-41 & 1876.91 & OT & $\mathrm{TH}$ & $2.39 \mathrm{E}-05$ & & & & 4 \\
\hline $144 \mathrm{R}-1,55-56$ & 1880.56 & OT & $\mathrm{AF}$ & $2.59 \mathrm{E}-05$ & & & & 4 \\
\hline $145 R-3,42-44$ & 1892.89 & OT & $\mathrm{TH}$ & $9.89 \mathrm{E}-06$ & & & & 4 \\
\hline 146R-2, 11-13 & 1900.31 & OT & $\mathrm{AF}$ & $6.56 \mathrm{E}-05$ & & & & 4 \\
\hline 146R-2, 11-13 & 1900.33 & $\mathrm{RM}$ & $\mathrm{TH}$ & & & & & 4 \\
\hline $147 R-5,123-125$ & 1915.35 & OT & $\mathrm{TH}$ & $2.29 \mathrm{E}-05$ & 27.20 & 12.2 & 210-or & 2 \\
\hline
\end{tabular}

$\mathrm{RM}=$ Rome laboratory, $\mathrm{OT}=$ Otago laboratory. $\mathrm{AF}=$ alternating field demagnetization, $\mathrm{TH}=$ thermal demagnetization. $\mathrm{NRM}=$ natural remanent magnetization, $\mathrm{ChRM}=$ characteristic remanent magnetization. $\mathrm{MAD}=$ maximum angular deviation. Steps: demagnetization steps included for ChRM calculation in $\mathrm{mT}$ for $\mathrm{AF}$ and ${ }^{\circ} \mathrm{C}$ for $\mathrm{TH}$, or $=$ anchored to the origin. Class = quality ranking for demagnetization data: $1=$ high, $2=$ intermediate quality, $3=$ unreliable, $4=$ no direction calculated. 
Table T3. Paleomagnetic demagnetization data of discrete samples, Hole U1353B.

\begin{tabular}{|c|c|c|c|c|c|c|c|c|}
\hline $\begin{array}{l}\text { Core, section, } \\
\text { interval }(\mathrm{cm})\end{array}$ & $\begin{array}{l}\text { Depth } \\
\text { CSF-A (m) }\end{array}$ & Lab & Treatment & $\begin{array}{c}\text { NRM } \\
\text { intensity } \\
(\mathrm{A} / \mathrm{m})\end{array}$ & $\begin{array}{c}\text { ChRM } \\
\text { inclination } \\
\left(^{\circ}\right)\end{array}$ & $\begin{array}{l}\text { MAD } \\
\left(^{\circ}\right)\end{array}$ & Steps & Class \\
\hline \multicolumn{9}{|l|}{ 317-U1353B- } \\
\hline $1 \mathrm{H}-1,53-55$ & 0.55 & RM & $\mathrm{AF}$ & $1.75 \mathrm{E}-03$ & & & & \\
\hline $1 \mathrm{H}-2,116-118$ & 2.68 & RM & $\mathrm{AF}$ & $1.04 \mathrm{E}-03$ & & & & 4 \\
\hline $1 \mathrm{H}-3,52-54$ & 3.54 & RM & $\mathrm{AF}$ & $9.92 \mathrm{E}-04$ & -36.4 & 30.3 & 25 -or & 4 \\
\hline $1 \mathrm{H}-4,114-116$ & 5.66 & RM & $\mathrm{AF}$ & $1.19 \mathrm{E}-03$ & -33.3 & 11.7 & 50 -or & 2 \\
\hline $1 \mathrm{H}-5,82-84$ & 6.84 & RM & $\mathrm{AF}$ & $1.20 \mathrm{E}-03$ & -43.6 & 13.2 & 45 -or & 3 \\
\hline $1 \mathrm{H}-6,50-52$ & 7.52 & RM & $\mathrm{AF}$ & $1.22 \mathrm{E}-03$ & -1.0 & 19.1 & 30 -or & 2 \\
\hline $2 \mathrm{H}-3,127-129$ & 12.29 & RM & $\mathrm{AF}$ & 1.17E-02 & & & & 3 \\
\hline $3 \mathrm{H}-1,111-113$ & 18.13 & RM & $\mathrm{AF}$ & $2.62 \mathrm{E}-04$ & -25.7 & 19.0 & 45 -or & 4 \\
\hline $3 \mathrm{H}-3,111-113$ & 21.13 & RM & $\mathrm{AF}$ & $2.41 \mathrm{E}-04$ & & & & 2 \\
\hline $3 \mathrm{H}-3,115-117$ & 21.17 & OT & $\mathrm{AF}$ & $6.05 \mathrm{E}-04$ & & & & 4 \\
\hline $3 \mathrm{H}-4,111-113$ & 22.63 & RM & $\mathrm{AF}$ & $3.91 \mathrm{E}-04$ & 46.9 & 12.8 & 40 -or & 4 \\
\hline $3 \mathrm{H}-5,50-52$ & 23.52 & RM & $\mathrm{AF}$ & $2.13 \mathrm{E}-04$ & & & & 1 \\
\hline $6 \mathrm{H}-2,124-126$ & 37.96 & RM & $\mathrm{AF}$ & $3.42 \mathrm{E}-04$ & 25.8 & 29.3 & 25 -or & 4 \\
\hline $6 \mathrm{H}-5,87-89$ & 42.09 & RM & $\mathrm{AF}$ & $7.38 \mathrm{E}-04$ & -44.5 & 36.6 & 45 -or & 3 \\
\hline 7H-1, 144-146 & 44.66 & RM & $\mathrm{AF}$ & $7.72 \mathrm{E}-04$ & -11.3 & 21.7 & 13 -or & 3 \\
\hline $7 \mathrm{H}-2,71-73$ & 45.43 & RM & $\mathrm{AF}$ & $2.27 \mathrm{E}-04$ & -68.7 & 7.2 & 21 -or & 3 \\
\hline $7 \mathrm{H}-4,49-51$ & 48.21 & RM & $\mathrm{AF}$ & $5.62 \mathrm{E}-04$ & -37.3 & 9.5 & 17 -or & 3 \\
\hline $8 \mathrm{H}-2,132-134$ & 51.74 & RM & $\mathrm{AF}$ & $3.26 \mathrm{E}-04$ & -40.9 & 10.9 & 21 -or & 2 \\
\hline $8 \mathrm{H}-5,50-52$ & 55.42 & RM & $\mathrm{AF}$ & 7.37E-04 & & & & 2 \\
\hline $8 \mathrm{H}-6,34-36$ & 56.26 & RM & $\mathrm{AF}$ & $8.02 \mathrm{E}-04$ & & & & 4 \\
\hline $9 \mathrm{H}-1,133-135$ & 58.15 & RM & $\mathrm{AF}$ & $7.00 \mathrm{E}-04$ & -18.2 & 14.4 & $17-25$ & 4 \\
\hline $9 \mathrm{H}-2,75-77$ & 59.07 & OT & $\mathrm{AF}$ & $1.05 \mathrm{E}-03$ & -67.9 & 6.5 & 15 -or & 3 \\
\hline $9 \mathrm{H}-3,44-46$ & 60.26 & RM & $\mathrm{AF}$ & $5.31 \mathrm{E}-04$ & -23.3 & 22.3 & 13 -or & 1 \\
\hline $10 \mathrm{H}-2,45-46$ & 63.96 & RM & $\mathrm{AF}$ & 7.91E-05 & -65.5 & 2.5 & 13 -or & 2 \\
\hline $10 \mathrm{H}-3,46-48$ & 65.48 & RM & $\mathrm{AF}$ & $8.66 \mathrm{E}-05$ & -59.9 & 6.6 & $17-30$ & 1 \\
\hline $10 \mathrm{H}-4,74-76$ & 67.26 & RM & $\mathrm{AF}$ & $3.66 \mathrm{E}-04$ & -13.8 & 10.9 & 13-or & 2 \\
\hline $11 \mathrm{H}-1,78-80$ & 68.40 & RM & $\mathrm{AF}$ & $1.35 \mathrm{E}-02$ & & & & 2 \\
\hline $11 \mathrm{H}-3,46-48$ & 71.08 & RM & $\mathrm{AF}$ & $3.14 \mathrm{E}-04$ & -20.2 & 9.5 & 21 -or & 4 \\
\hline $11 \mathrm{H}-4,47-49$ & 72.59 & RM & $\mathrm{AF}$ & $1.68 \mathrm{E}-04$ & -46.3 & 13.8 & $13-30$ & 3 \\
\hline $12 \mathrm{H}-3,111-113$ & 76.31 & RM & $\mathrm{AF}$ & $1.04 \mathrm{E}-04$ & -69.8 & 22.4 & 13-or & 3 \\
\hline $18 X-1,81-83$ & 107.43 & От & $\mathrm{AF}$ & $1.26 \mathrm{E}-03$ & & & & 2 \\
\hline $18 X-1,123-125$ & 107.85 & RM & $\mathrm{AF}$ & $7.18 \mathrm{E}-04$ & 50.8 & 17.4 & 21 -or & 4 \\
\hline $19 \mathrm{H}-4,109-111$ & 115.79 & RM & $\mathrm{AF}$ & $3.45 \mathrm{E}-04$ & 35.3 & 23.1 & 25 -or & 2 \\
\hline $23 \mathrm{H}-2,128-130$ & 133.00 & RM & $\mathrm{AF}$ & $3.11 \mathrm{E}-04$ & 39.5 & 14.2 & 30 -or & 2 \\
\hline $23 \mathrm{H}-3,78-80$ & 134.00 & OT & $\mathrm{AF}$ & $2.99 \mathrm{E}-04$ & 38.8 & 19.6 & 30 -or & 2 \\
\hline $24 \mathrm{H}-2,48-50$ & 136.34 & OT & $\mathrm{AF}$ & $8.85 \mathrm{E}-04$ & & & & 2 \\
\hline $25 \mathrm{H}-2,32-34$ & 142.04 & OT & $\mathrm{AF}$ & $1.86 \mathrm{E}-03$ & & & & 4 \\
\hline $26 \mathrm{H}-1,128-130$ & 143.70 & RM & $\mathrm{AF}$ & $1.36 \mathrm{E}-03$ & 42.9 & 24.9 & 25 -or & 4 \\
\hline $26 \mathrm{H}-2,105-107$ & 144.97 & OT & $\mathrm{AF}$ & $1.05 \mathrm{E}-03$ & 49.3 & 7.7 & 20 -or & 2 \\
\hline $29 \mathrm{H}-1,65-67$ & 153.67 & OT & $\mathrm{AF}$ & $3.85 \mathrm{E}-03$ & & & & 2 \\
\hline $29 \mathrm{H}-4,30-32$ & 155.20 & $\mathrm{RM}$ & $\mathrm{AF}$ & $8.12 \mathrm{E}-04$ & 63.8 & 19.9 & 30 -or & 4 \\
\hline $39 \mathrm{H}-2,77-79$ & 194.85 & OT & $\mathrm{AF}$ & $3.77 \mathrm{E}-03$ & -44.6 & 3.3 & 35 -or & 2 \\
\hline $40 \mathrm{H}-2,68-70$ & 198.60 & OT & $\mathrm{AF}$ & $1.74 \mathrm{E}-03$ & & & & 2 \\
\hline $45 \mathrm{H}-2,85-87$ & 218.47 & OT & $\mathrm{AF}$ & $1.99 \mathrm{E}-04$ & & & & 4 \\
\hline $46 \mathrm{H}-2,113-115$ & 222.21 & OT & $\mathrm{AF}$ & $6.37 \mathrm{E}-04$ & & & & 4 \\
\hline $57 \mathrm{H}-2,12-14$ & 252.84 & $\mathrm{RM}$ & $\mathrm{AF}$ & 6.67E-04 & 38.7 & 9.9 & 30 -or & 4 \\
\hline $58 \mathrm{H}-1,74-76$ & 254.36 & OT & $\mathrm{AF}$ & $3.78 \mathrm{E}-04$ & & & & 1 \\
\hline $63 X-1,69-71$ & 270.31 & OT & $\mathrm{AF}$ & $1.07 \mathrm{E}-02$ & & & & 4 \\
\hline $64 X-1,35-37$ & 279.57 & $\mathrm{RM}$ & $\mathrm{AF}$ & $2.21 \mathrm{E}-04$ & & & & 4 \\
\hline $65 X-1,73-75$ & 289.55 & OT & $\mathrm{AF}$ & $7.74 \mathrm{E}-04$ & 33.6 & 7.3 & 25 -or & 4 \\
\hline $68 x-1,60-62$ & 318.22 & $\mathrm{RM}$ & $\mathrm{AF}$ & $8.26 \mathrm{E}-05$ & 34.7 & 7.2 & 13-or & 2 \\
\hline $69 X-1,33-35$ & 327.55 & RM & $\mathrm{AF}$ & $1.96 \mathrm{E}-04$ & & & & 2 \\
\hline $71 X-1,55-57$ & 346.57 & OT & $\mathrm{AF}$ & $2.60 \mathrm{E}-04$ & 40.6 & 12.5 & 15 -or & 4 \\
\hline $88 X-1,42-44$ & 510.04 & $\mathrm{RM}$ & $\mathrm{AF}$ & $1.87 \mathrm{E}-04$ & 21.8 & 10.9 & 25 -or & 2 \\
\hline
\end{tabular}

$\mathrm{RM}=$ Rome laboratory, OT = Otago laboratory. $\mathrm{AF}=$ alternating field demagnetization. $\mathrm{NRM}=$ natural remanent magnetization, $\mathrm{ChRM}=\mathrm{charac}-$ teristic remanent magnetization. MAD = maximum angular deviation. Steps: demagnetization steps included for ChRM calculation in mT for AF, or $=$ anchored to the origin. Class $=$ quality ranking for demagnetization data: $1=$ high, $2=$ intermediate quality, $3=$ unreliable, $4=$ no direction calculated. 
Table T4. Paleomagnetic demagnetization data of discrete samples, Holes U1354B and U1354C. (Continued on next page.)

\begin{tabular}{|c|c|c|c|c|c|c|c|c|}
\hline $\begin{array}{l}\text { Core, section, } \\
\text { interval }(\mathrm{cm})\end{array}$ & $\begin{array}{c}\text { Depth } \\
\text { CSF-A (m) }\end{array}$ & Lab & Treatment & $\begin{array}{c}\text { NRM } \\
\text { intensity } \\
(\mathrm{A} / \mathrm{m})\end{array}$ & $\begin{array}{c}\text { ChRM } \\
\text { inclination } \\
\left(^{\circ}\right)\end{array}$ & $\begin{array}{l}\text { MAD } \\
\left({ }^{\circ}\right)\end{array}$ & Steps & Class \\
\hline \multicolumn{9}{|l|}{ 317-U1354B- } \\
\hline $1 \mathrm{H}-1,126-128$ & 1.28 & $\mathrm{RM}$ & $\mathrm{AF}$ & $7.74 \mathrm{E}-04$ & -22.07 & 28.0 & & 3 \\
\hline $1 \mathrm{H}-2,49-51$ & 2.01 & RM & $\mathrm{AF}$ & $8.08 \mathrm{E}-04$ & -51.65 & 13.6 & 17-or & 2 \\
\hline $1 \mathrm{H}-2,111-113$ & 2.63 & OT & $\mathrm{AF}$ & $2.59 \mathrm{E}-03$ & & & 25 -or & 4 \\
\hline $1 \mathrm{H}-3,7-9$ & 3.09 & $\mathrm{RM}$ & $\mathrm{AF}$ & $9.94 \mathrm{E}-04$ & 54.29 & 19.4 & & 3 \\
\hline $2 \mathrm{H}-1,97-99$ & 5.09 & RM & $\mathrm{AF}$ & $1.01 \mathrm{E}-03$ & 30.21 & 17.7 & 17-or & 3 \\
\hline $2 \mathrm{H}-2,97-99$ & 6.59 & RM & $\mathrm{AF}$ & $7.71 \mathrm{E}-04$ & -81.55 & 23.6 & 17-or & 3 \\
\hline $2 \mathrm{H}-3,94-96$ & 8.06 & RM & $\mathrm{AF}$ & $6.85 \mathrm{E}-04$ & & & 17-or & 4 \\
\hline $2 \mathrm{H}-4,97-99$ & 9.59 & RM & $\mathrm{AF}$ & $2.69 \mathrm{E}-03$ & -30.36 & 25.7 & & 3 \\
\hline $4 \mathrm{H}-2,49-51$ & 14.11 & $\mathrm{RM}$ & $\mathrm{AF}$ & $3.05 \mathrm{E}-04$ & -71.01 & 11.5 & $17-45$ & 2 \\
\hline $4 \mathrm{H}-3,53-55$ & 15.65 & RM & $\mathrm{AF}$ & $1.12 \mathrm{E}-04$ & -44.28 & 25.1 & 21 -or & 3 \\
\hline $4 \mathrm{H}-4,73-75$ & 17.35 & OT & $\mathrm{AF}$ & $4.61 \mathrm{E}-04$ & -21.8 & 11.1 & 21 -or & 2 \\
\hline $5 \mathrm{H}-1,60-62$ & 18.32 & RM & $\mathrm{AF}$ & $3.08 \mathrm{E}-04$ & -14.57 & 34.6 & 20 -or & 3 \\
\hline $8 \mathrm{H}-1,125-127$ & 27.87 & RM & $\mathrm{AF}$ & $3.44 \mathrm{E}-04$ & -12.58 & 33.2 & 17-or & 3 \\
\hline $8 \mathrm{H}-2,77-79$ & 28.89 & RM & $\mathrm{AF}$ & $3.89 \mathrm{E}-04$ & -12.58 & 33.2 & 21 -or & 3 \\
\hline $8 \mathrm{H}-2,77-79$ & 28.89 & $\mathrm{RM}$ & $\mathrm{AF}$ & $1.22 \mathrm{E}-04$ & -26.02 & 11.6 & 21 -or & 3 \\
\hline $8 \mathrm{H}-3,80-82$ & 30.42 & OT & $\mathrm{AF}$ & $1.14 \mathrm{E}-03$ & & & 25 -or & 4 \\
\hline $8 \mathrm{H}-4,101-103$ & 32.13 & $\mathrm{RM}$ & $\mathrm{AF}$ & $2.75 \mathrm{E}-04$ & -47.17 & 24.4 & & 3 \\
\hline $8 \mathrm{H}-5,30-32$ & 32.92 & RM & $\mathrm{AF}$ & $2.99 \mathrm{E}-04$ & 19.95 & 16.2 & $13-25$ & 2 \\
\hline $9 \mathrm{H}-1,75-77$ & 34.27 & RM & $\mathrm{AF}$ & $4.80 \mathrm{E}-04$ & & & 17-or & 4 \\
\hline $9 \mathrm{H}-3,54-56$ & 37.06 & OT & $\mathrm{AF}$ & $1.32 \mathrm{E}-03$ & & & & 4 \\
\hline $9 \mathrm{H}-4,81-83$ & 38.83 & RM & $\mathrm{AF}$ & $5.06 \mathrm{E}-04$ & -27.36 & 23.3 & & 3 \\
\hline $9 \mathrm{H}-5,82-84$ & 40.34 & $\mathrm{RM}$ & $\mathrm{AF}$ & $2.96 \mathrm{E}-04$ & & & $17-30$ & 4 \\
\hline $9 \mathrm{H}-6,34-36$ & 40.96 & RM & $\mathrm{AF}$ & $4.26 \mathrm{E}-04$ & & & & 4 \\
\hline $10 \mathrm{H}-1,76-78$ & 42.28 & RM & $\mathrm{AF}$ & $6.46 \mathrm{E}-03$ & -28.31 & 23.5 & & 3 \\
\hline $10 \mathrm{H}-2,75-77$ & 43.77 & RM & $\mathrm{AF}$ & $3.46 \mathrm{E}-04$ & & & 17-or & 4 \\
\hline $10 \mathrm{H}-3,73-75$ & 45.25 & RM & $\mathrm{AF}$ & $2.04 \mathrm{E}-04$ & -22.73 & 20.6 & & 3 \\
\hline $10 \mathrm{H}-5,61-63$ & 48.13 & RM & $\mathrm{AF}$ & $5.48 \mathrm{E}-04$ & -35.42 & 15.3 & 21 -or & 2 \\
\hline $10 \mathrm{H}-6,77-79$ & 49.79 & RM & $\mathrm{AF}$ & $4.10 \mathrm{E}-04$ & -54.51 & 6.5 & 13-or & 1 \\
\hline $11 \mathrm{H}-1,116-118$ & 51.68 & $\mathrm{RM}$ & $\mathrm{AF}$ & $8.38 \mathrm{E}-04$ & -48.73 & 12.9 & 17-or & 1 \\
\hline $11 \mathrm{H}-2,121-123$ & 53.23 & $\mathrm{RM}$ & $\mathrm{AF}$ & $1.00 \mathrm{E}-03$ & -48.03 & 21.7 & 40 -or & 2 \\
\hline $11 \mathrm{H}-3,115-117$ & 54.67 & RM & $\mathrm{AF}$ & $5.25 \mathrm{E}-04$ & -42.75 & 4.1 & 25 -or & 1 \\
\hline $11 \mathrm{H}-4,80-82$ & 55.82 & RM & $\mathrm{AF}$ & $6.20 \mathrm{E}-04$ & -22.55 & 26.4 & 17-or & 2 \\
\hline $11 \mathrm{H}-5,73-75$ & 57.25 & RM & $\mathrm{AF}$ & 1.47E-03 & -51.52 & 13.8 & 13-or & 1 \\
\hline $12 \mathrm{H}-2,47-49$ & 58.91 & RM & $\mathrm{AF}$ & $8.39 \mathrm{E}-04$ & & & 35 -or & 4 \\
\hline $12 \mathrm{H}-3,128-130$ & 61.22 & OT & $\mathrm{AF}$ & $5.72 \mathrm{E}-04$ & -40.6 & 1.8 & & 3 \\
\hline $13 \mathrm{H}-1,101-103$ & 63.53 & OT & $\mathrm{AF}$ & 8.67E-04 & & & 10 -or & 4 \\
\hline $13 \mathrm{H}-2,12-14$ & 64.14 & $\mathrm{RM}$ & $\mathrm{AF}$ & $3.18 \mathrm{E}-04$ & 61.17 & 19.8 & & 1 \\
\hline $13 \mathrm{H}-2,118-120$ & 65.20 & $\mathrm{RM}$ & $\mathrm{AF}$ & $2.62 \mathrm{E}-04$ & 36.39 & 12.9 & 35 -or & 2 \\
\hline $13 \mathrm{H}-3,119-121$ & 66.71 & OT & $\mathrm{AF}$ & $1.80 \mathrm{E}-03$ & & & 40 -or & 4 \\
\hline $13 \mathrm{H}-4,93-95$ & 67.95 & RM & $\mathrm{AF}$ & $5.08 \mathrm{E}-04$ & 45.16 & 14.5 & & 2 \\
\hline $13 \mathrm{H}-5,73-75$ & 69.25 & $\mathrm{RM}$ & $\mathrm{AF}$ & $4.12 \mathrm{E}-04$ & 61.40 & 6.6 & 21 -or & 1 \\
\hline $13 \mathrm{H}-6,20-22$ & 69.81 & RM & $\mathrm{AF}$ & $7.30 \mathrm{E}-04$ & 59.50 & 20.5 & 21 -or & 2 \\
\hline $14 \mathrm{H}-1,109-111$ & 71.61 & $\mathrm{RM}$ & $\mathrm{AF}$ & $3.34 \mathrm{E}-04$ & 65.18 & 15.2 & 35 -or & 2 \\
\hline $14 \mathrm{H}-2,21-23$ & 72.23 & $\mathrm{RM}$ & $\mathrm{AF}$ & $3.44 \mathrm{E}-04$ & 52.18 & 15.7 & 30 -or & 1 \\
\hline $15 \mathrm{H}-1,45-47$ & 73.97 & $\mathrm{RM}$ & $\mathrm{AF}$ & $3.62 \mathrm{E}-04$ & & & 17-or & 4 \\
\hline \multicolumn{9}{|l|}{ 317-U1354C- } \\
\hline $2 \mathrm{H}-1,120-122$ & 66.22 & $\mathrm{RM}$ & $\mathrm{AF}$ & $3.32 \mathrm{E}-04$ & 61.82 & & & 2 \\
\hline $2 \mathrm{H}-2,120-122$ & 67.72 & RM & $\mathrm{AF}$ & $4.79 \mathrm{E}-04$ & 58.96 & 12.4 & 17-or & 1 \\
\hline $2 \mathrm{H}-4,102-105$ & 70.55 & $\mathrm{RM}$ & $\mathrm{AF}$ & $2.81 \mathrm{E}-04$ & 64.01 & 12.3 & 35 -or & 1 \\
\hline $2 \mathrm{H}-5,86-88$ & 71.88 & RM & $\mathrm{AF}$ & 3.37E-04 & 50.38 & 10.9 & 17-or & 2 \\
\hline $4 X-1,86-88$ & 78.98 & $\mathrm{RM}$ & $\mathrm{AF}$ & $6.96 \mathrm{E}-04$ & 53.45 & 29.4 & 40 -or & 1 \\
\hline $4 \mathrm{X}-2,123-125$ & 80.85 & $\mathrm{RM}$ & $\mathrm{AF}$ & $2.21 \mathrm{E}-04$ & 40.41 & 6.1 & 21 -or & 1 \\
\hline $5 X-3,45-47$ & 85.77 & RM & $\mathrm{AF}$ & $3.03 \mathrm{E}-04$ & 51.72 & 8.3 & 21 -or & 1 \\
\hline $5 X-3,95-97$ & 86.27 & RM & $\mathrm{AF}$ & $3.49 \mathrm{E}-04$ & 69.56 & 1.6 & 30 -or & 2 \\
\hline $6 X-3,134-136$ & 96.26 & RM & $\mathrm{AF}$ & $1.95 \mathrm{E}-04$ & 75.60 & 15.4 & 30 -or & 1 \\
\hline $7 X-1,84-86$ & 102.36 & $\mathrm{RM}$ & $\mathrm{AF}$ & $2.09 \mathrm{E}-03$ & 80.26 & 6.3 & 40 -or & 2 \\
\hline $7 X-3,110-112$ & 105.62 & $\mathrm{RM}$ & $\mathrm{AF}$ & $1.06 \mathrm{E}-03$ & 55.99 & 2.9 & 40 -or & 1 \\
\hline $8 X-3,21-23$ & 109.73 & $\mathrm{RM}$ & $\mathrm{AF}$ & $4.51 \mathrm{E}-04$ & 56.61 & 7.1 & 25 -or & 2 \\
\hline $9 X-1,43-45$ & 116.55 & $\mathrm{RM}$ & $\mathrm{AF}$ & $3.03 \mathrm{E}-04$ & -67.11 & 10.9 & 25 -or & 2 \\
\hline $9 X-2,124-126$ & 118.86 & $\mathrm{RM}$ & $\mathrm{AF}$ & $1.59 \mathrm{E}-04$ & -47.93 & 17.9 & 21 -or & 2 \\
\hline $9 X-4,21-23$ & 120.83 & $\mathrm{RM}$ & $\mathrm{AF}$ & $6.49 \mathrm{E}-05$ & -5.83 & 14.7 & 17-or & 3 \\
\hline $10 X-2,117-119$ & 128.29 & RM & $\mathrm{AF}$ & 1.57E-04 & -40.94 & 16.5 & 35 -or & 1 \\
\hline $10 X-4,33-35$ & 130.45 & RM & $\mathrm{AF}$ & $7.35 \mathrm{E}-05$ & 27.27 & 12.5 & 21 -or & 1 \\
\hline $10 X-5,113-115$ & 132.75 & RM & $\mathrm{AF}$ & $8.00 \mathrm{E}-05$ & 14.91 & 12.7 & 17-or & 2 \\
\hline $11 X-1,46-48$ & 135.58 & $\mathrm{RM}$ & $\mathrm{AF}$ & 1.17E-04 & -64.70 & 10.6 & 17-or & 2 \\
\hline
\end{tabular}


Table T4 (continued).

\begin{tabular}{|c|c|c|c|c|c|c|c|c|}
\hline $\begin{array}{c}\text { Core, section, } \\
\text { interval }(\mathrm{cm})\end{array}$ & $\begin{array}{c}\text { Depth } \\
\text { CSF-A (m) }\end{array}$ & Lab & Treatment & $\begin{array}{c}\text { NRM } \\
\text { intensity } \\
(\mathrm{A} / \mathrm{m})\end{array}$ & $\begin{array}{c}\text { ChRM } \\
\text { inclination } \\
\left(^{\circ}\right)\end{array}$ & $\begin{array}{c}\text { MAD } \\
\left({ }^{\circ}\right)\end{array}$ & Steps & Class \\
\hline $11 X-2,46-48$ & 137.08 & $\mathrm{RM}$ & $\mathrm{AF}$ & $3.98 \mathrm{E}-05$ & -32.95 & 13.4 & 35 -or & 2 \\
\hline $11 X-4,27-29$ & 139.89 & $\mathrm{RM}$ & $\mathrm{AF}$ & $1.85 \mathrm{E}-04$ & & 8.4 & 17-or & 4 \\
\hline $11 X-5,29-31$ & 141.41 & $\mathrm{RM}$ & $\mathrm{AF}$ & $3.50 \mathrm{E}-04$ & & & & 4 \\
\hline $11 X-6,44-46$ & 143.06 & $\mathrm{RM}$ & $\mathrm{AF}$ & $1.55 \mathrm{E}-04$ & & & & 4 \\
\hline $11 X-7,20-22$ & 143.82 & $\mathrm{RM}$ & $\mathrm{AF}$ & $2.78 \mathrm{E}-04$ & -9.70 & & & 1 \\
\hline $12 X-2,97-99$ & 147.19 & $\mathrm{RM}$ & $\mathrm{AF}$ & $5.38 \mathrm{E}-04$ & 75.40 & 5.8 & 17-or & 1 \\
\hline $12 X-3,105-107$ & 148.77 & $\mathrm{RM}$ & $\mathrm{AF}$ & $3.74 \mathrm{E}-04$ & 78.78 & 2.3 & 40 -or & 1 \\
\hline $12 X-5,51-53$ & 151.23 & $\mathrm{RM}$ & $\mathrm{AF}$ & $1.15 \mathrm{E}-03$ & 11.92 & 3.8 & 30 -or & 1 \\
\hline $12 X-6,30-32$ & 152.02 & $\mathrm{RM}$ & $\mathrm{AF}$ & $1.31 \mathrm{E}-03$ & -54.55 & 5.1 & 40 -or & 1 \\
\hline $13 X-1,88-90$ & 155.20 & $\mathrm{RM}$ & $\mathrm{AF}$ & $7.21 \mathrm{E}-04$ & -42.85 & 2.0 & 35 -or & 2 \\
\hline $13 X-2,115-117$ & 156.97 & $\mathrm{RM}$ & $\mathrm{AF}$ & 7.57E-04 & 29.91 & 15.8 & 25 -or & 1 \\
\hline $13 X-5,26-28$ & 160.58 & RM & $\mathrm{AF}$ & $1.01 \mathrm{E}-04$ & -10.62 & 19.3 & 25 -or & 3 \\
\hline $14 X-2,55-57$ & 165.89 & $\mathrm{RM}$ & $\mathrm{AF}$ & $6.40 \mathrm{E}-05$ & 67.67 & 12.2 & 35 -or & 1 \\
\hline $15 X-1,107-109$ & 174.59 & $\mathrm{RM}$ & $\mathrm{AF}$ & $7.69 \mathrm{E}-04$ & 39.77 & 1.1 & 35 -or & 1 \\
\hline $15 X-2,104-106$ & 176.06 & $\mathrm{RM}$ & $\mathrm{AF}$ & $1.28 \mathrm{E}-04$ & 15.33 & 8.0 & 30 -or & 2 \\
\hline $15 X-4,92-94$ & 178.94 & $\mathrm{RM}$ & $\mathrm{AF}$ & $4.78 \mathrm{E}-05$ & 29.17 & 8.5 & 17-or & 2 \\
\hline $18 X-1,72-74$ & 203.04 & RM & $\mathrm{AF}$ & $4.09 \mathrm{E}-05$ & 45.91 & 14.3 & 17-or & 3 \\
\hline $18 X-3,92-94$ & 206.24 & $\mathrm{RM}$ & $\mathrm{AF}$ & $2.28 \mathrm{E}-04$ & -39.90 & 10.0 & 21 -or & 1 \\
\hline $18 X-4,40-42$ & 207.12 & $\mathrm{RM}$ & $\mathrm{AF}$ & $1.76 \mathrm{E}-03$ & -45.32 & 7.3 & 30 -or & 1 \\
\hline $19 X-1,48-50$ & 212.30 & $\mathrm{RM}$ & $\mathrm{AF}$ & $1.51 \mathrm{E}-03$ & -12.53 & 13.4 & 17-or & 2 \\
\hline $19 X-3,89-91$ & 215.71 & $\mathrm{RM}$ & $\mathrm{AF}$ & $3.55 \mathrm{E}-04$ & -60.74 & 5.4 & 13-or & 2 \\
\hline $21 X-1,80-82$ & 231.82 & RM & $\mathrm{AF}$ & $6.74 \mathrm{E}-05$ & -60.74 & 16.2 & 30 -or & 2 \\
\hline $21 X-1,80-82$ & 231.82 & $\mathrm{RM}$ & $\mathrm{AF}$ & $2.71 \mathrm{E}-04$ & & 16.2 & 30 -or & 4 \\
\hline $21 X-3,115-117$ & 235.17 & $\mathrm{RM}$ & $\mathrm{AF}$ & $5.60 \mathrm{E}-05$ & -32.32 & & & 3 \\
\hline $21 X-4,67-69$ & 236.19 & $\mathrm{RM}$ & $\mathrm{AF}$ & $1.03 \mathrm{E}-04$ & 45.74 & 20.2 & 17-or & 3 \\
\hline $21 X-5,87-89$ & 237.89 & $\mathrm{RM}$ & $\mathrm{AF}$ & $2.06 \mathrm{E}-04$ & -10.60 & 7.7 & 35 -or & 1 \\
\hline 21X-6, 99-101 & 239.51 & RM & $\mathrm{AF}$ & $5.52 \mathrm{E}-05$ & & 7.1 & 13-or & 4 \\
\hline $22 X-3,118-120$ & 244.70 & $\mathrm{RM}$ & $\mathrm{AF}$ & $1.25 \mathrm{E}-04$ & 46.04 & & & 2 \\
\hline $26 X-1,32-34$ & 279.24 & $\mathrm{RM}$ & $\mathrm{AF}$ & $7.59 \mathrm{E}-04$ & & 20.0 & $21-o r$ & 4 \\
\hline $27 X-1,95-97$ & 289.47 & $\mathrm{RM}$ & $\mathrm{AF}$ & $2.50 \mathrm{E}-04$ & -38.71 & & & 2 \\
\hline $27 X-3,107-109$ & 292.54 & $\mathrm{RM}$ & $\mathrm{AF}$ & $7.68 \mathrm{E}-05$ & & 14.7 & 45 -or & 4 \\
\hline
\end{tabular}

$\mathrm{RM}=$ Rome laboratory, OT = Otago laboratory. $\mathrm{AF}=$ alternating field demagnetization. $\mathrm{NRM}=$ natural remanent magnetization, $\mathrm{ChRM}=\mathrm{charac}-$ teristic remanent magnetization. MAD = maximum angular deviation. Steps: demagnetization steps included for ChRM calculation in mT for AF, or $=$ anchored to the origin. Class $=$ quality ranking for demagnetization data: $1=$ high, $2=$ intermediate quality, $3=$ unreliable, $4=$ no direction calculated. 